\title{
ON THE SPREAD OF CERTAIN NORMAL MATRICES
}

\author{
A THESIS \\ Submitted to the Faculty of Graduate Studies and Research \\ In Partial Fulfillment of the Requirements \\ FOR THE DEgREE OF \\ Doctor OF PHILOSOPHY \\ IN \\ Mathematics \\ UNIVERSITY OF REGINA
}

By

Yongjun Xing

Regina, Saskatchewan

June 2011

(C) Copyright 2011: Yongjun Xing 
Library and Archives

Canada

Published Heritage

Branch

395 Wellington Street

Ottawa ON K1A ON4

Canada
Bibliothèque et

Archives Canada

Direction du

Patrimoine de l'édition

395 , rue Wellington

Ottawa ON K1A ON4

Canada
Your file Votre référence

ISBN: 978-0-494-88593-2

Our file Notre référence

ISBN: $978-0-494-88593-2$

\section{NOTICE:}

The author has granted a nonexclusive license allowing Library and Archives Canada to reproduce, publish, archive, preserve, conserve, communicate to the public by telecommunication or on the Internet, loan, distrbute and sell theses worldwide, for commercial or noncommercial purposes, in microform, paper, electronic and/or any other formats.

The author retains copyright ownership and moral rights in this thesis. Neither the thesis nor substantial extracts from it may be printed or otherwise reproduced without the author's permission.
AVIS:

L'auteur a accordé une licence non exclusive permettant à la Bibliothèque et Archives Canada de reproduire, publier, archiver, sauvegarder, conserver, transmettre au public par télécommunication ou par l'Internet, prêter, distribuer et vendre des thèses partout dans le monde, à des fins commerciales ou autres, sur support microforme, papier, électronique et/ou autres formats.

L'auteur conserve la propriété du droit d'auteur et des droits moraux qui protege cette thèse. $\mathrm{Ni}$ la thèse ni des extraits substantiels de celle-ci ne doivent être imprimés ou autrement reproduits sans son autorisation.
In compliance with the Canadian Privacy Act some supporting forms may have been removed from this thesis.

While these forms may be included in the document page count, their removal does not represent any loss of content from the thesis.
Conformément à la loi canadienne sur la protection de la vie privée, quelques formulaires secondaires ont été enlevés de cette thèse.

Bien que ces formulaires aient inclus dans la pagination, il n'y aura aucun contenu manquant. 


\section{UNIVERSITY OF REGINA}

\section{FACULTY OF GRADUATE STUDIES AND RESEARCH SUPERVISORY AND EXAMINING COMMITTEE}

Yongjun Xing, candidate for the degree of Doctor of Philosophy in Mathematics, has presented a thesis titled, On the Spread of Certain Normal Matrices, in an oral examination held on June 8, 2011. The following committee members have found the thesis acceptable in form and content, and that the candidate demonstrated satisfactory knowledge of the subject material.

External Examiner: $\quad$ *Dr. Fuzhen Zhang, Nova Southeastern University

Supervisor:

Dr. Shaun Fallat, Department of Mathematics and Statistics

Committee Member:

*Dr. Douglas Farenick, Department of Mathematics and Statistics

Committee Member:

Dr. Chun-Hua Guo, Department of Mathematics and Statistics

Committee Member:

Dr. Boting Yang, Department of Computer Science

Chair of Defense:

Dr. Howard Hamilton, Department of Computer Science

*Not present at defense 


\section{Abstract}

A spread of a matrix has extensive and practical applications in some combinatorial optimization problems and cybernetics problems. The spread of a matrix is simply defined as the maximum absolute value of the difference between any two eigenvalues of that matrix. There are many existing papers dealing with bounding the spread of a matrix in general. Of interest to us is the spread of $n \times n$ normal matrices with entries in a closed set. In this thesis, we are interested in the classes of real symmetric matrices, real skew-symmetric matrices, complex Hermitian matrices and complex skew-Hermitian matrices, and we determine the structure of these matrices, in each class, when their spread attains a maximum value. Matlab is used as a tool to aid the verification of some cases. Motivated by some existing results about eigenvalue inclusion regions via discs, we try to build a connection among the disc coverings, spread, and principal submatrices. 


\section{Acknowledgements}

I would like to acknowledge the strong and helpful support of my supervisor $\mathrm{Dr}$. Shaun M. Fallat. Without his help and patience, this work would never have been brought to a conclusion. I would like to acknowledge the generous financial support of the Department of Mathematics and Statistics at the University of Regina, and of the Faculty of Graduate Studies and Research at the University of Regina. I would like to acknowledge the careful reading and the helpful suggestions from my committee. 


\section{Contents}

$\begin{array}{ll}\text { Abstract } & \text { i }\end{array}$

Acknowledgements $\quad$ ii

Table of Contents $\quad$ iii

$\begin{array}{ll}\text { List of Figures } & \text { vi }\end{array}$

1 Introduction 1

1.1 The Spread of a Matrix (History and Motivation) . . . . . . . . 1

1.2 Notation and Terminology .................. 3

1.3 Outline of the Thesis . . . . . . . . . . . . 6

2 Preliminary Results $\quad 8$

2.1 Known Results .................... 8

2.1 .1 Background ..................... 8

2.1.2 Lower Bounds for the Spread of a Normal Matrix . . . . . . . 9

2.1.3 Upper Bounds for the Spread of a Graph . . . . . . . . . . 14

2.2 Consequences, Facts and Other Results . . . . . . . . . . . . 25 
2.2 .1 Mirsky's Theorem . . . . . . . . . . . . . . . . 25

3 Maximum Spread for Certain Classes of Normal Matrices 29

3.1 Case of Real Normal Matrices . . . . . . . . . . . . 29

3.2 Real Symmetric Matrices . . . . . . . . . . . . . . . 35

3.2.1 Structure of Real Symmetric Matrices Whose Spreads Attain the Bound from Mirsky's Theorem $\ldots \ldots \ldots$

3.3 Spread of Some Real Symmetric Matrices $\ldots \ldots \ldots \ldots \ldots$

3.3.1 The Form of Real Symmetric Matrices of Rank 2, with Entries

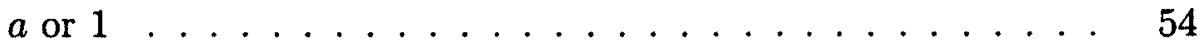

3.3.2 The Spread of Real Symmetric Rank 2 Matrices with Entries $a$

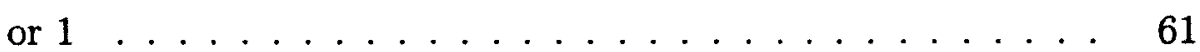

3.4 Conclusion about Real Symmetric Matrices . . . . . . . . . . . 74

3.4.1 Maximum Spread of Real Symmetric Rank 2 Matrices with Entries in the Interval $[a, 1] \quad(-1 \leq a<1) \ldots \ldots \ldots 74$

3.4.2 Maximum Spread of Real Symmetric Matrices with Entries in the Interval $[a, b] \ldots \ldots \ldots \ldots \ldots \ldots \ldots$

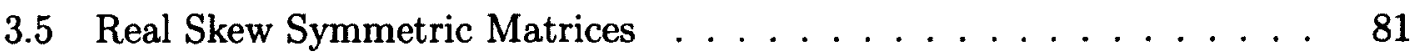

3.5.1 Structure of Real Skew Symmetric Matrices Whose Spreads Attain the Bound in the Mirsky's Theorem $\ldots \ldots$. . . 85

3.6 Case of Complex Normal Matrices . . . . . . . . . . . . . 97 
3.6.1 Structure of Complex Hermitian Matrices Whose Spreads Attain the Bound in Mirsky's Theorem . . . . . . . . . . 97

3.6.2 Complex Hermitian Matrices with Entries of Modulus 1 . . . 102

3.6.3 Skew Hermitian Matrices with Entries of Modulus No More $\operatorname{than} 1 \ldots \ldots \ldots \ldots \ldots$

4 Spectral Inclusions for Normal Matrices 106

4.1 New Spectral Inclusions for Normal Matrices . . . . . . . . . . . 106

4.2 Imbedding Conditions for Normal Matrices . . . . . . . . . . . . 112

4.3 The Spread of a Normal Matrix and Its Principal Submatrices . . . . 116

5 Conclusion $\quad 119$

5.1 Summary ................................ 119

5.2 Future Considerations . . . . . . . . . . . . . . 121

$\begin{array}{lll}6 & \text { Appendix } & 123\end{array}$

6.1 The Matlab Programs ... . . . . . . . . . . . . 123

$\begin{array}{ll}\text { References } & 128\end{array}$ 


\section{List of Figures}

3.1 Two cases of the combination parameters in real skew symmetric matrices 86

3.2 Case for both arguments in quadrant I . . . . . . . . . 86

3.3 Subcases 1 and $2 \ldots \ldots \ldots \ldots$ 


\section{Chapter 1}

\section{Introduction}

The central theme in this dissertation is to investigate the maximum spread of certain normal matrices. We will introduce necessary notation and preliminary material followed by an outline of this dissertation. In addition, we will give brief introductions within each of the chapters.

\subsection{The Spread of a Matrix (History and Motivation)}

The spread of a matrix has been of research interest for many mathematicians since the 1950's. There is considerable literature on the spread of an arbitrary matrix, see, e.g., Mirsky (1956); Johnson et al.(1985); Thompson (1992); Nylen and Tam (1994); Zhan (2006). This quantity has applications in combinatorial optimization problems [8]. Recent research has shown a connection to graph theory. In Petrović (1983), Petrović determined all minimal graphs whose spreads do not exceed 4 . In Gregory et al. (2001), Gregory, Hershkowitz, and Kirkland presented some lower and upper bounds for the spread of a graph, and show that the unique graph with minimum 
spread among all connected graphs of a given order is the path. However the graph(s) with maximum spread is still unclear, but some conjectures on this topic appear in the paper [9]. In Nikiforov (2006), Nikiforov considers properties of linear combinations of some extreme eigenvalues of a graph. He presents a theorem involving the limit of certain combinations as the order of a graph goes to infinity, and gives an upper bound for the sum of the first two largest eigenvalue of all graphs of fixed order. In $\mathrm{Li}$ et al. (2007), Li, Zhang, and Zhou determine the unique graph with maximum spread among all unicyclic graphs on at least 18 vertices. Such a graph is obtained from a star by adding an edge between two pendant vertices. In Fan et al. (2008), Fan, $\mathrm{Xu}$, Wang, and Liang show that the star is the unique tree with maximum Laplacian spread among all trees of given order, and the path is the unique one with minimal Laplacian spread among all trees of given order.

Generally speaking, the spread of a square matrix $A$ with order $n$ is defined as

$$
S P(A)=\max _{i, j}\left|\lambda_{i}-\lambda_{j}\right|
$$

where $\lambda_{i}, \lambda_{j}$ are eigenvalues of $A, 1 \leq i<j \leq n$. When all the eigenvalues are real, $S P(A)=\lambda_{1}-\lambda_{n}$, where the eigenvalues of $A$ are ordered as $\lambda_{1} \geq \lambda_{2} \geq \cdots \geq \lambda_{n}$.

The study of normal matrices is another research field for mathematicians because this class of matrices has a series of beautiful properties, such as unitary diagonalization. Some papers investigate the spread of a normal matrix, like [26, 27]. However, there is little research involving the spread of a normal matrix whose entries are restricted in some range. Our main purpose is to investigate the maximum spread of a certain normal matrices whose entries are in an interval or a circle, and determine 
the structure of a corresponding extreme normal matrix.

\subsection{Notation and Terminology}

As mentioned previously, let $A \in \mathbb{C}^{n \times n}$, the spread of $A$ is defined as $S P(A)=$ $\max _{i, j}\left|\lambda_{i}-\lambda_{j}\right|$, where $\lambda_{i}, \lambda_{j}$ are eigenvalues of $A, 1 \leq i<j \leq n$; when all the eigenvalues are real, $S P(A)=\lambda_{1}-\lambda_{n}$, assuming that the eigenvalues of $A$ are ordered as $\lambda_{1} \geq \lambda_{2} \geq \cdots \geq \lambda_{n}$. Denote the set of eigenvalues of $A \in \mathbb{C}^{n \times n}$ as $\sigma(A) ;$ and denote $\Lambda(A)$, set of singular values of matrix $A$, which are the square roots of the eigenvalues of the nonnegative Hermitian matrix $A A^{*}$, where $A^{*}$ is the conjugate transpose of $A$.

A complex $n \times n$ matrix $A$ is a normal matrix if $A A^{*}=A^{*} A$, i.e, a normal matrix commutes with its conjugate transpose. If $A$ is a real normal matrix, then $A^{T} A=A A^{T}$, where $A^{T}$ is the transpose of $A$.

Denote $\operatorname{tr}(A)$ as the trace of the matrix $A$, i.e. $\operatorname{tr}(A)=\sum_{i=1}^{n} a_{i i}, A=\left[a_{i j}\right] \in \mathbb{C}^{n \times n}$, and denote $\|A\|_{F}$ as the Frobenius norm of $A$, i.e.

$$
\|A\|_{F}^{2}=\operatorname{tr}\left(A^{*} A\right)=\sum_{1 \leq i, j \leq n}\left|a_{i j}\right|^{2}
$$

Let $x, y \in \mathbb{C}^{n}, x=\left(x_{1}, x_{2}, \ldots, x_{n}\right)^{T}, y=\left(y_{1}, y_{2}, \ldots, y_{n}\right)^{T}$. Then denote the inner product of vectors $(x, y)=y^{*} x=\sum_{i=1}^{n} \bar{y}_{i} x_{i}$.

Let

$$
W(A)=\{(x, A x):(x, x)=1\}
$$

denote the numerical range of matrix $A$. When $A$ is a normal matrix, $W(A)$ is the convex hull of the spectrum of $A$, denoted $\operatorname{co}(\sigma(A))$. 
Let $A=\left[a_{i j}\right] \in \mathbb{C}^{n \times n}, B=\left[b_{i j}\right] \in \mathbb{C}^{n \times n}$, denote $C=A \circ B=\left[c_{i j}\right] \in \mathbb{C}^{n \times n}$, where $c_{i j}=a_{i j} \times b_{i j}$. This product is called the Schur (or Hadamard) product of $A$ and $B$.

A simple graph is an undirected graph that has no loops and no more than one edge between any two different vertices. A complete graph is a simple graph in which every pair of distinct vertices is connected by a unique edge. A bipartite graph (or bigraph) is a graph whose vertices can be divided into two disjoint sets $V_{1}$ and $V_{2}$ such that every edge connects a vertex in $V_{1}$ to one in $V_{2}$; that is, $V_{1}$ and $V_{2}$ are independent sets. A complete bipartite graph, $G:=\left(V_{1} \cup V_{2}, E\right)$, where $V_{1}, V_{2}$ are the sets of vertices and $E$ the set of edges of $G$, is a bipartite graph such that for any two vertices, $v_{1} \in V_{1}$ and $v_{2} \in V_{2}, v_{1} v_{2}$ is an edge in $G$. The complete bipartite graph with partitions of size $\left|V_{1}\right|=a$ and $\left|V_{2}\right|=b$, is denoted by $K_{a, b}$.

The degree of a vertex $v_{i}$ of a graph is the number of edges incident to the vertex, and is denoted by $d_{i}$. Let $G$ be a simple graph of order $n$ with degree sequence $d_{1}, d_{2}, \ldots, d_{n}$. Define $M_{1}=\sum_{i=1}^{n} d_{i}^{2}$.

The Laplacian matrix of a graph $G$ is defined as $L=L(G)=D(G)-A(G)$, where $D(G)=\operatorname{diag}\left\{d_{1}, \ldots, d_{n}\right\}$ is a diagonal matrix with the degree of a vertex $v_{i}$ of $G$ as the diagonal entries, $A(G)$ is the adjacency $n \times n$ matrix of $\mathrm{G}$ given by: $a_{i j}=1$ if vertices $v_{i}, v_{j}$ are adjacent and $a_{i j}=0$ otherwise. The Laplacian spread of the graph $G$ is defined as $S P(G)=\lambda_{1}(G)-\lambda_{n-1}(G)$, since the eigenvalues of $L$ can be arranged as follows: $\lambda_{1}(G) \geq \lambda_{2}(G) \geq \cdots \geq \lambda_{n}(G)=0$, where $\lambda_{1}(G)$ is the spectral radius of $L$, and $\lambda_{n-1}(G)$ is called algebraic connectivity.

For a real number $a$, let $a^{+}$equal $a$ if $a \geq 0$ and 0 otherwise. 
For $\alpha, \beta, \gamma \in \mathbb{F}^{n}$, if there exist $u, v \in \mathbb{F}$ such that $\gamma=u \alpha+v \beta$, then we call this pair, $(u, v)$, the combination parameters of $\alpha, \beta$.

Denote $\operatorname{round}(x)$ an integer number closest to $x$. Examples for clarity: $\operatorname{round}(1.5)=$ 1 or $2 ; \operatorname{round}(2.2)=2$.

Denote $\operatorname{rank}(A)$ the rank of a matrix $A$.

Let $J_{n}$ denote a matrix of order $n$, whose entries are all ones, and $e_{n \times 1}=$ $[1, \ldots, 1]^{T} \in \mathbb{R}^{n}$, let $N_{n}[a, b]$ denote the set of $n \times n$ normal matrices all of whose entries lie in the interval $[a, b]$, and let $S_{n}[a, b]$ denote the set of $n \times n$ real symmetric matrices, contained in $N_{n}[a, b]$. Let $\mathscr{U}_{n}$ denote the set of unitary matrices, i.e, for any matrix $U \in \mathscr{U}_{n}, U U^{*}=U^{*} U=I$. For an $n \times n$ real symmetric matrix $A$, we always denote the eigenvalues of $A$ in decreasing order, $\lambda_{1}(A) \geq \cdots \geq \lambda_{n}(A)$.

Denote by $S_{n}-\{a, b\}$ as the set of $n \times n$ real symmetric matrices whose entries are either $a$ or $b$.

Denote by $S S_{n}[-a, a]$ the set of $n \times n$ real skew symmetric matrices with entries in the interval $[-a, a]$.

Let $\mathscr{H}_{n}$ be the class of $n \times n$ Hermitian matrices and $\mathscr{S} \mathscr{H}_{n}$ the class of $n \times n$ skew Hermitian matrices.

Two matrices $A, B$, with the same order, are said to be $D$-similar if there is a diagonal matrix $D$ with diagonal entries equal to 1 or -1 such that $D A D=B$.

In Chapter 2, we define a special class of matrices, called Mirsky matrices. A Mirsky matrix $A$ is a matrix which attains the bound in (2.25) and therefore has the property that $n-2$ eigenvalues coincide and are all equal to the arithmetic mean of 
the remaining two eigenvalues of $A$, and $A$ is normal.

In Chapter 4 , we denote by $A_{i}$ the $(n-1) \times(n-1)$ principal submatrix of $A$ obtained by deleting the $i$-th row and column from $A$.

Let $A=\left[a_{i j}\right] \in N_{n}$. Define

$$
r_{i}=\left[\sum_{j=1, j \neq i}^{n}\left|a_{i j}\right|^{2}\right]^{1 / 2}
$$

and let $D(\alpha, r)$ be the closed disk with radius $r \in \mathbb{R}^{+}$and center $\alpha \in \mathbb{C}$.

For real $\theta$, define $\leq_{\theta}$ as the total order with positive cone $e^{i \theta} H$, where

$$
H=\{a+i b: a>0 \text {, or } a=0 \text { and } b>0\} .
$$

In this case, the lexicographic order is $\leq_{0}$, and we have

$$
\alpha \leq_{\theta} \beta \Leftrightarrow e^{-i \theta} \alpha \leq_{0} e^{-i \theta} \beta
$$

\subsection{Outline of the Thesis}

This dissertation is organized into five chapters: In Chapter 1 (this chapter), we present some background and notation. In Chapter 2, we introduce some results on the upper bounds and lower bounds of spread for a general matrix and a graph and then we survey preliminary results and related facts. In Chapter 3, we show the main contribution: maximum spread of normal matrices with entries in an interval or a circle and discuss the related structure of the extreme matrices. In Chapter 4 , we review Borcea's conjectures on the eigenvalues of a matrix being covered by the circles of the related principal submatrices and we give some conjectures about the 
rough upper bound of the spread for the matrices with entries in some range, and then we survey the relation between a normal matrix and its principal submatrices. In Chapter 5, we summarize the main results in this thesis and discuss some possible future work in this area. Finally, an appendix is included containing the algorithms for finding a Mirsky matrix and a normal matrix with entries in some given range when spread is maximum. 


\section{Chapter 2}

\section{Preliminary Results}

\subsection{Known Results}

In this chapter, we review some results on lower and upper bounds of the spread for general normal matrices and some simple graphs, and we present a key theorem, called Mirsky's Theorem, which inspires our investigation of the maximum spread of a normal matrix with entries in a fixed range.

\subsubsection{Background}

In the thesis, we focus on the class of normal matrices. Recall that a complex square matrix $A$ is a normal matrix if

$$
A A^{*}=A^{*} A
$$

where $A^{*}$ is the conjugate transpose of $A$. That is, a matrix is normal if it commutes with its conjugate transpose. If $A$ is a real matrix, then $A^{*}=A^{T}$; it is normal if $A^{T} A=A A^{T}$ 


\subsubsection{Lower Bounds for the Spread of a Normal Matrix}

In this section, we introduce some classic lower bounds for the spread of a normal matrix.

Recall that of $x, y \in \mathbb{C}^{n}$, then $(x, y)$ denotes the inner product of $x$ and $y$.

Theorem 2.1.1 ([20]) If $A$ is normal, then

$$
\begin{aligned}
S P(A) & =\sup _{u, v}|(u, A u)-(v, A v)| \\
& \geq \sqrt{3} \sup _{u, v}|(u, A v)| .
\end{aligned}
$$

If, in addition $A$ is Hermitian, then

$$
S P(A)=2 \sup _{u, v}|(u, A v)|
$$

where the upper bounds above are taken with respect to all orthonormal vectors $u, v$.

If we let

$$
W(A)=\{(x, A x):(x, x)=1\}
$$

denote the numerical range of $A$, then it is well known that for normal matrices, $W(A)$ is the convex hull of the spectrum of $A$, denoted $\operatorname{co}(\sigma(A))$, which is the minimal convex set containing all the eigenvalues of $A$. Thus the equality in (2.1) follows, and moreover the supremum is attained if we choose $u$ and $v$ to be the eigenvectors corresponding to the eigenvalues for which the maximum spread is attained. In fact, it is clear that this relation holds whenever $W(A)=\operatorname{co}(\sigma(A))$. A characterization of such matrices $A$ is given in [13]. 
By compactness and continuity, the supremum is attained in (2.1) as well. In [10] it is shown that, for any $A$,

$$
\sup _{\substack{\|u\|=\|v\|=1 \\(u, v)=0}}|(u, A v)|=\min _{\alpha}\|A-\alpha I\|_{s p}
$$

where $\|\cdot\|_{s p}$ denotes spectral norm.

In particular, there are other lower bounds known, and some are presented below. For example,

$$
S P(A) \geq \frac{1}{n-1}\left|\sum_{i \neq j} a_{i j}\right|
$$

and, if $R_{i}$ denotes the $i^{\text {th }}$ row sum, $\nu$ denotes the standard deviation of the row sums, and $R_{i_{1}} \geq R_{i_{2}} \geq \cdots \geq R_{i_{n}}$, then (for $A$ symmetric)

$$
S P(A) \geq 2 \nu \geq\left\{\frac{2}{n} \sum_{j=1}^{\lceil(n+1) / 2\rceil}\left(R_{i_{j}}-R_{i_{n-j+1}}\right)^{2}\right\}^{1 / 2} \geq \frac{2}{n} \sum_{j=1}^{\lceil(n+1) / 2\rceil}\left(R_{i_{j}}-R_{i_{n-j+1}}\right)
$$

where $\lceil\cdot\rceil$ denotes the greatest integer part.

Mirsky $[19,20]$ presented the following lower bounds for $S P(A)$, when $A$ is normal:

$$
\begin{aligned}
& S P(A) \geq \sqrt{3} \max _{i \neq j}\left|a_{i j}\right|, \\
& S P(A) \geq \max _{i \neq j}\left\{\left(\operatorname{Re} a_{i i}-\operatorname{Re} a_{j j}\right)^{2}+\left|a_{i j}+\bar{a}_{j i}\right|^{2}\right\}^{1 / 2}, \\
& S P(A) \geq \max _{i \neq j}\left\{\left|a_{i i}-a_{j j}\right|^{2}+\left(\left|a_{i j}\right|-\left|a_{j i}\right|\right)^{2}\right\}^{1 / 2}, \\
& S P(A) \geq \max _{i \neq j}\left(\left|a_{i j}\right|+\left|a_{j i}\right|\right), \\
& S P(A) \geq \max _{i \neq j}\left(\frac{1}{2} c_{i j}\right)^{1 / 2},
\end{aligned}
$$

where $c_{i j}=\left|a_{i i}-a_{j j}\right|^{2}+\left|\left(a_{i i}-a_{j j}\right)^{2}+4 a_{i j} a_{j i}\right|+2\left|a_{i j}\right|^{2}+2\left|a_{j i}\right|^{2}$. 
If $A$ is Hermitian, then:

$$
\begin{aligned}
& S P(A) \geq 2 \max _{i \neq j}\left|a_{i j}\right|, \\
& S P(A) \geq \max _{i \neq j}\left\{\left(a_{i i}-a_{j j}\right)^{2}+4\left|a_{i j}\right|^{2}\right\}^{1 / 2} .
\end{aligned}
$$

Brauer and Mewborn [2] presented the following lower bounds for $S P(A)$ for $A$ normal. If $n \geq 2$, let $s_{1}$ be the trace of any principal submatrix $B$ of order $k \geq 3$, and $s_{2}$ the sum of the principal minors of order 2 of $B$. Then

$$
S P(A) \geq \begin{cases}\frac{2}{k}\left|(k-1) s_{1}^{2}-2 k s_{2}\right|^{1 / 2}, & k \text { even } \\ \left(\frac{4}{k^{2}-1}\right)^{1 / 2}\left|(k-1) s_{1}^{2}-2 k s_{2}\right|^{1 / 2}, & k \text { odd }\end{cases}
$$

Let $c_{1}$ be the sum of all the eigenvalues of $A$ and $c_{2}$ be the sum of the product of any two different eigenvalues of $A$ respectively. Set $K_{2}=\left\{2(1-1 / n) c_{1}^{2}-4 c_{2}\right\}^{1 / 2}$. If $A$ has real roots, then

$$
S P(A) \geq \begin{cases}\sqrt{\frac{2}{n}} K_{2}, & n \text { even } \\ \left(\frac{2 n}{n^{2}-1}\right)^{1 / 2} K_{2}, & n \text { odd }\end{cases}
$$

With $A$ Hermitian,

$$
\begin{aligned}
S P(A) & \geq \frac{1}{2} \max _{i \neq j}\left\{a_{i i}+a_{j j}+\left[\left(a_{i i}-a_{j j}\right)^{2}+4\left|a_{i j}\right|^{2}\right]^{1 / 2}\right\} \\
& -\frac{1}{2} \min _{i \neq j}\left\{a_{i i}+a_{j j}-\left[\left(a_{i i}-a_{j j}\right)^{2}+4\left|a_{i j}\right|^{2}\right]^{1 / 2}\right\}
\end{aligned}
$$

and if, in addition, $n \geq 3, s_{1}$ is the trace of any principal submatrix $B$ of order $k \geq 3$, and $s_{2}$ is the sum of the principal minors of order 2 of $B$, then

$$
S P(A) \geq \begin{cases}\frac{2}{k}\left\{(k-1) s_{1}^{2}-2 k s_{2}\right\}^{1 / 2}, & k \text { even } \\ \left(\frac{4}{k^{2}-1}\right)^{1 / 2}\left\{(k-1) s_{1}^{2}-2 k s_{2}\right\}^{1 / 2}, & k \text { odd }\end{cases}
$$


Wolkowicz and Styan [31] presented lower bounds for matrices $A$ with real engenvalues. Let

$$
s^{2}=\frac{\operatorname{tr}\left(A^{2}\right)}{n}-\left(\frac{\operatorname{tr}(A)}{n}\right)^{2}
$$

then

$$
S P(A) \geq \begin{cases}2 s, & n \text { even, } \\ 2 s n /\left(n^{2}-1\right)^{1 / 2}, & n \text { odd } .\end{cases}
$$

Lower bounds for general matrices are given in [32].

Considering other choices for the orthonormal vectors $u$ and $v$ in (2.1) and (2.2), Johnson et al. presented the following lower bound for the spread of a real and normal matrix $A$.

Theorem 2.1.2 ([16]) Suppose that $A$ is real and normal (or more generally $W(A)=$ $\operatorname{co}(\sigma(A))$. Then

$$
S P(A) \geq \frac{1}{n-1}\left|\sum_{i \neq j} a_{i j}\right| .
$$

Proof. Choose $u=\frac{1}{\sqrt{n}} e_{n \times 1}$ and $v=\frac{1}{\sqrt{2}}\left(e_{k}-e_{l}\right)$, where $e_{k}$ and $e_{l}$ are the $k^{\text {th }}$ and $l^{\text {th }}$ standard basis vectors, respectively. Then (2.1) implies that

$$
\begin{aligned}
S P(A) & \geq \frac{1}{n} \sum_{i, j} a_{i j}-\min _{k \neq l}\left(\frac{a_{k k}+a_{l l}-2 a_{k l}}{2}\right) \\
& \geq \frac{1}{n} \sum_{i, j} a_{i j}-\frac{1}{2 n(n-1)} \sum_{k \neq l}\left(a_{k k}+a_{l l}\right)+\frac{1}{n(n-1)} \sum_{k \neq l} a_{k l} \\
& =\frac{1}{n} \sum_{i, j} a_{i j}-\frac{1}{2 n(n-1)} \sum_{k} 2(n-1) a_{k k}+\frac{1}{n(n-1)} \sum_{k \neq l} a_{k l} \\
& =\frac{1}{n-1} \sum_{i \neq j} a_{i j} .
\end{aligned}
$$


The result now follows by noting that $S P(-A)=S P(A)$. 


\subsubsection{Upper Bounds for the Spread of a Graph}

First we introduce some results about upper bounds for the spread, $S P(G)$, of a graph. The matrix $A$ here is related to the graph and is real and symmetric, and is known as the adjacency matrix of a simple graph $G$ with $n$ vertices. So $S P(G)=S P(A)=\lambda_{1}-\lambda_{n}$, if we assume the eigenvalues of $A$ are $\lambda_{1} \geq \lambda_{2} \geq \cdots \geq \lambda_{n}$. The following theorem on $S P(G)$ is due to Gregory et al. [9].

Theorem 2.1.3 For a graph $G$ with $n$ vertices and e edges,

$$
S P(G) \leq \lambda_{1}+\sqrt{2 e-\lambda_{1}^{2}} \leq 2 \sqrt{e} .
$$

If $G$ has no isolated vertices, then equality holds throughout if and only if equality holds in the first inequality; equivalently, if and only if $G=K_{a, b}$ for some $a, b$ with $e=a b$ and $a+b \leq n$.

Remark 1: A complete graph is a simple graph in which every pair of distinct vertices is connected by a unique edge. Geometrically $K_{3}$ forms the edge set of a triangle, $K_{4}$ a tetrahedron, etc.

Remark 2: A complete bipartite graph, $G:=\left(V_{1}+V_{2}, E\right)$, where $V_{1}, V_{2}$ are the sets of vertices and $E$ the set of edges of $G$, is a bipartite graph such that for any two vertices, $v_{1} \in V_{1}$ and $v_{2} \in V_{2}, v_{1} v_{2}$ is an edge in $G$. The complete bipartite graph with partitions of size $\left|V_{1}\right|=a$ and $\left|V_{2}\right|=b$, is denoted $K_{a, b}$.

Gregory, Hershkowitz, and Kirkland give other results for the maximum spread of a graph with $e$ edges and a graph with $n$ vertices. They consider a complete $p$-partite graph (precisely $p$ non-empty parts), which is a simple graph (a simple graph is an 
undirected graph that has no loops and no more than one edge between any two different vertices).

For the first case, maximum spread of a graph with $e$ edges, they have the following,

Theorem 2.1.4 ([9]) Let $G$ be a graph with $n$ vertices, e edges, and precisely $k$ negative eigenvalues, $1 \leq k \leq n-1$. Then

$$
S P(G) \leq \frac{k+1}{k} \lambda_{1}+\sqrt{2 e \frac{k-1}{k}-\frac{k^{2}-1}{k^{2}} \lambda_{1}^{2}} .
$$

Equality holds if and only if $G$ has at most three distinct non-zero eigenvalues: $\lambda_{1}, \beta, \lambda_{n}$, where $\lambda_{1}>0>\beta \geq \lambda_{n}$ and $\beta$ has multiplicity $k-1$ if $\beta>\lambda_{n}$ and multiplicity $k$ if $\beta=\lambda_{n}$. Equivalently, equality holds if and only if $G_{0}$ is a complete $(k+1)$-partite graph and, when $k+1 \geq 4$, the $k$ smallest parts of $G_{0}$ all have equal size (necessarily, $\left.-\beta=\left(\lambda_{1}+\lambda_{n}\right) /(k-1)\right)$.

They prove the above theorem for the values of $k=1,2$ and $k \geq 3$ using the characteristic polynomial of the adjacency matrix of $G$. We can see that the partial derivative with respect to $k$ of the upper bound in the theorem above is non-negative for all real $k \geq 2$. Consequently, the inequality in the theorem above holds if $G$ has at least 2 and at most $k$ negative eigenvalues. And the partial derivative with respect to $\lambda_{1}$ of the upper bound in the theorem above is

$$
\frac{k+1}{k}\left(1-\frac{(k-1) \lambda_{1}}{k \Delta}\right)
$$

where $\Delta$ is the square root in (2.14). It implies that the upper bound in Theorem 2.1.4 is strictly increasing in $\lambda_{1}$ when $\lambda_{1} \leq \sqrt{e}$ and strictly decreasing when $\lambda_{1} \geq \sqrt{e}$. 
They then present the following corollary.

Corollary 2.1.5 ([9]) Let $G$ be a graph with $n$ vertices, $e>\left\lfloor n^{2} / 4\right\rfloor$ edges, and at most $k$ negative eigenvalues. Then

$$
S P(G) \leq \frac{k+1}{k}\left(\frac{2 e}{n}\right)+\sqrt{2 e \frac{k-1}{k}-\frac{k^{2}-1}{k^{2}}\left(\frac{2 e}{n}\right)^{2}} .
$$

Equality holds if and only if $(k+1) \mid n$ and $G$ is a regular complete $(k+1)$-partite graph.

Since $e>\left\lfloor n^{2} / 4\right\rfloor, \lambda_{1} \geq \frac{2 e}{n}>\sqrt{e}$, we can derive the inequality above with replacing $\lambda_{1}$ by $\frac{2 e}{n}$. Equality holds if and only if equality holds in Theorem 2.1 .4 and $\lambda_{1}=\frac{2 e}{n}$, which means that $G$ is a regular and a complete $(k+1)$-partite graph.

For the second case, $S P(n)$, the maximum possible spread of a graph with $n$ vertices, they have some upper bounds.

By studying the relation between $H$, an induced subgraph of $G$ on $n-1$ vertices and $G$, a connected graph on $n$ vertices, they find the next lemma.

Lemma 2.1.6 ([9]) If $H$ is an induced subgraph of $G$, then $\lambda_{n}(G) \leq \lambda_{n}(H) \leq$ $\lambda_{1}(H) \leq \lambda_{1}(G)$. Thus $S P(G) \geq S P(H)$ with strict inequality if $G$ is connected and $H$ is a proper induced subgraph of $G$.

It follows that $S P(n)$ is strictly increasing, and they present a conjecture about the maximum spread $S P(n)$.

Conjecture 2.1.7 ([9]) The maximum spread $S P(n)$ of the graphs of order $n$ is attained only by $G(n,\lfloor 2 n / 3\rfloor)$; that is, $S P(n)=\left\lfloor(4 / 3)\left(n^{2}-n+1\right)\right\rfloor^{1 / 2}$ and so $(1 / \sqrt{3})(2 n-1)<S P(n)<(1 / \sqrt{3})(2 n-1)+\sqrt{3} /(4 n-2)$. 
Then they verify that $G=G(n,\lfloor 2 n / 3\rfloor)$ if $G$ is one of the these following graphs on $n$ vertices and $G$ has maximum spread, according to the value of $k$.

If $k=1$, then $G$ is a complete bipartite and so, by Theorem 2.1.3, $S P(G)=$ $2 \sqrt{e} \leq n<S P(n)$ when $n \geq 3$, and $G=K_{2}=G(n,\lfloor 2 n / 3\rfloor)$ when $n=2$.

If $k=2$, then $G$ is a (complete) tripartite graph and Proposition 2.1.9 (see below) implies that $S P(G)<S P(n)$ when $n \geq 35$.

If $k \geq 3$, the extremal graphs $G$ in Theorem 2.1.4 are the complete $(k+1)$-partite graphs with $k$ parts of size $n_{1}$ and a single part of size $n_{2} \geq n_{1}$. Denote such a graph by $G\left(n, k, n_{1}\right)$. For this case, they first show that $n_{1}=1$. Since $S P(n)$ is strictly increasing, only graphs without isolated vertices need to be examined. Thus, $n=k n_{1}+n_{2}$. It follows from the discussion in the proof of Theorem 2.1.4 that the extreme eigenvalues $\lambda_{1}, \lambda_{n}$ of $G\left(n, k, n_{1}\right)$ are the zeros of the middle factor of the characteristic polynomial of (the adjacency matrix of) $G[4, \mathrm{p} .74]$ given by

$$
\lambda^{n-k-1}\left(1-\sum_{i=1}^{t} \frac{n_{i} m_{i}}{\lambda+n_{i}}\right) \prod_{i=1}^{t}\left(\lambda+n_{i}\right)^{m_{i}}
$$

or, equivalently, they are the roots of the quadratic equation $\lambda^{2}-n_{1}(k-1) \lambda-k n_{1} n_{2}=$ 0 . Thus, $G\left(n, k, n_{1}\right)$ has spread

$$
\lambda_{1}-\lambda_{n}=\left(n_{1}^{2}(k-1)^{2}+4 k n_{1} n_{2}\right)^{1 / 2}
$$

Since $k n_{1}=n-n_{2}$ and $n_{1}(k-1) \leq n-n_{2}-1$ with equality if and only if $n_{1}=1$, it follows that, for $k \geq 3$, the spread is largest when $n_{1}=1$, as required. When $n_{1}=1, G(n, k, 1)=G(n, k)$. Taking $n_{1}=1$ in $(2.15)$ gives $S P(G(n, k))=\left((k-1)^{2}+\right.$ $4 k(n-k))^{1 / 2}$. This quadratic is maximum when $k$ is an integer closest to $(2 n-1) / 3$, 
that is, when $k=\lfloor 2 n / 3\rfloor$, as claimed at the outset.

Gregory et al. [9] present some necessary conditions that a graph $G$ on $n$ vertices must satisfy when it attains the maximum spread $S P(n)$.

Lemma 2.1.8 ([9]) Let $G$ be a graph with e edges and let $\alpha \geq 1$. If $S P(G)>\alpha n$, then e must satisfy the quadratic inequality $8 e^{2}-(4 \alpha+2) n^{2} e+\alpha^{2} n^{2}<0$.

Proposition 2.1.9 ([9]) If $S P(G)=S P(n)$ and $n \geq 35$, then $G$ is not tripartite.

Proposition 2.1.10 ([9]) If $S P(G)=S P(n)$, then $G$ must be connected.

They then describe some characteristics about the pair of eigenvectors for the extreme eigenvalues when the maximum spread is attained. For a simple graph $G$ of order $n$, the adjacency matrix $A$ is symmetric. So

$$
\lambda_{1} \geq x^{T} A x \text { and } \lambda_{n} \leq y^{T} A y,
$$

where $\lambda_{1} \geq \lambda_{2} \geq \cdots \geq \lambda_{n}, \lambda_{i} \in \sigma(A), i=1, \ldots, n$, and unit vectors $x, y \in \mathbb{R}^{n}$. Equalities hold if and only if $x, y$ are the unit eigenvectors associated with $\lambda_{1}, \lambda_{n}$ respectively. Thus

$$
S P(G)=\max _{x, y} \sum_{i, j} a_{i, j}\left(x_{i} x_{j}-y_{i} y_{j}\right),
$$

where the maximum is taken over all pairs of unit vectors in $\mathbb{R}^{n}$ and is attained only for orthonormal eigenvectors of $A$ corresponding to the eigenvalues $\lambda_{1}, \lambda_{n}$, respectively. The entries of $x$ may always be assumed to be non-negative (and positive if $G$ is connected). Define such an ordered pair of orthonormal eigenvectors $x, y$ of $G$ with $x \geq 0$ (i.e, $x$ entry-wise nonnegative) an extremal pair of eigenvectors of $G$. 
The following three lemmas are an immediate consequence of (2.16) and Proposition 2.1.10.

Lemma 2.1.11 ([9]) Suppose $S P(G)=S P(n)$ and $x, y$ is an extremal pair of eigenvectors of $G$. Then distinct vertices $i, j$ of $G$ must be adjacent whenever $x_{i} x_{j}-y_{i} y_{j} \geq 0$ and non-adjacent whenever $x_{i} x_{j}-y_{i} y_{j}<0$.

Lemma 2.1.12 ([9]) $S P(n)=S P\left((G(x, y))\right.$ for some graph $G(x, y)$ with $x, y \in \mathbb{R}^{n}$ orthonormal and $x$ positive.

For a real number $a$, let $a^{+}$equal $a$ if $a \geq 0$ and 0 otherwise.

Lemma 2.1.13 ([9]) For $n \geq 2, S P(n)=\max \sum_{i, j}\left(x_{i} x_{j}-y_{i} y_{j}\right)^{+}$, where the maximum is taken over all pairs $x, y$ of orthonormal vectors in $\mathbb{R}^{n}$ with $x$ entry-wise positive.

As we know, in graph theory, the degree of a vertex of a graph is the number of edges incident to the vertex. The degree of a vertex $v_{i}$ is denoted $d_{i}$ here.

Liu et al. [18] provide an improvement of Gregory's bound on $S P(G)$ for graphs with additional restrictions as followings. Let $G$ be a simple graph of order $n$ with degree sequence $d_{1}, d_{2}, \ldots, d_{n}$. Define $M_{1}=\sum_{i=1}^{n} d_{i}^{2}$. Then the following lemma is a description about $M_{1}$. We call $G$ an $(n, e)$ graph if $G$ has $n$ vertices and e edges.

Lemma 2.1.14 ([18]) For any $(n, e)$ graph with $f$ 4-cycles,

$$
M_{1}=\frac{1}{2} \operatorname{tr}\left(A^{4}\right)+e-4 f
$$


where $A$ is an adjacency matrix of $G$. Furthermore, if $\lambda_{1}, \lambda_{2}, \ldots, \lambda_{n}$ are the eigenvalues of $A$, then

$$
M_{1}=\frac{1}{2} \sum_{i=1}^{n} \lambda_{i}^{4}+e-4 f
$$

Proof. Let $A=\left(a_{i j}\right)$ and $A^{k}=\left(a_{i j}^{k}\right)$. It is known (see [4]) that $a_{i i}^{4}$ is the number of all closed walks of length 4 from vertex $i$ to $i$ in $G$. Let $f_{i}$ denote the number of 4-cycles located at vertex $i$. Denote $i \sim j$ as vertex $i$ is adjacent to vertex $j$. Then

$$
\begin{aligned}
a_{i i}^{4} & =d_{i}^{2}+\sum_{j \sim i}\left(d_{j}-1\right)+2 f_{i} \\
& =d_{i}^{2}+\sum_{j \sim i} d_{j}-d_{i}+2 f_{i} .
\end{aligned}
$$

Thus,

$$
\begin{aligned}
\operatorname{tr}\left(A^{4}\right) & =\sum_{i=1}^{n} a_{i i}^{4} \\
& =\sum_{i=1}^{n} d_{i}^{2}+\sum_{i=1}^{n} \sum_{j \sim i} d_{j}-\sum_{i=1}^{n} d_{i}+2 \sum_{i=1}^{n} f_{i} \\
& =M_{1}+M_{1}-2 e+8 f .
\end{aligned}
$$

Hence $M_{1}=\frac{1}{2} \operatorname{tr}\left(A^{4}\right)+e-4 f$, as $\sum_{i=1}^{n} \lambda_{i}^{4}=\operatorname{tr}\left(A^{4}\right)$, trace of $A^{4}$ (see [4]).

They then focus on graphs without $K_{4}^{\prime} s$ (called $K_{4}$-free graphs).

Lemma 2.1.15 ([18]) For a $K_{4}$-free $(n, e)$ graph $G, M_{1} \leq e^{2}+e-4 f$. If $G$ is connected and equality holds, then $|N(u) \cup N(v)|=n$ holds for any $\{u, v\} \in E(G)$. 
Proof. For any edge $\{u, v\} \in E(G)$, let $f_{u v}$ denote the number of 4-cycles containing edge $\{u, v\}$. Since $G$ is a $K_{4}$-free graph, then $d(u)+d(v) \leq e+1-f_{u v}$. Thus,

$$
\sum_{u \sim v}(d(u)+d(v)) \leq \sum_{u \sim v} e+\sum_{u \sim v} 1-\sum_{u \sim v} f_{u v} .
$$

It follows that $M_{1} \leq e^{2}+e-4 f$.

If equality holds in this lemma, by inequality (2.17), it follows that $d(u)+d(v)=$ $e+1-f_{u v}$ holds for any $\{u, v\} \in E(G)$. Let $G^{\prime}$ denote the subgraph induced by $N(u) \cup N(v)$, and $e^{\prime}$ denote the number of edges of $G^{\prime}$. Since $G$ (and then $G^{\prime}$ ) is $K_{4}$-free, then $e+1-f_{u v}=d(u)+d(v) \leq e^{\prime}+1-f_{u v} \leq e+1-f_{u v}$, implies that $e=e^{\prime}$. Moreover, since $G$ is connected, then $|N(u) \cup N(v)|=n$.

The following corollary implies that if $\sum_{i=1}^{n} \lambda_{i}^{4}>2 e^{2}$, then $G$ contains at least one $K_{4}$.

Corollary 2.1.16 ([18]) For a $K_{4}$-free $(n, e)$ graph, $\sum_{i=1}^{n} \lambda_{i}^{4} \leq 2 e^{2}$, where equality holds for $G \cong K_{a, b}$ or $G \cong K_{3}$.

Proof. Combining Lemma 2.1.14 and 2.1.15, we have

$$
\frac{1}{2} \sum_{i=1}^{n} \lambda_{i}^{4}+e-4 f \leq e^{2}+e-4 f .
$$

Hence, $\sum_{i=1}^{n} \lambda_{i}^{4} \leq 2 e^{2}$. If $G \cong K_{a, b}$ or $G \cong K_{3}$, it is easy to check that $d(u)+d(v)=$ $e+1-f_{u v}$ holds for any $u, v \in E(G)$. Thus, equality holds in inequality (2.17), implies that equality holds. 
Let $\operatorname{rank}(A)$ the rank of a matrix $A$. Now the following is from [18] on the upper bound for the spread of a graph.

Theorem 2.1.17 For an $(n, e)$ graph $G$ with eigenvalues $\lambda_{1}, \lambda_{2}, \ldots, \lambda_{n}$,

$$
S P(G) \leq \lambda_{1}+\sqrt[4]{2 M_{1}-2 e+8 f-\lambda_{1}^{4}} \leq 2 \sqrt[4]{M_{1}-e+4 f}
$$

If $G$ has no isolated vertices, then equality holds throughout if and only if equality holds in the first inequality; equivalently, if and only if $G=K_{a, b}$ for some $a, b$ with $e=a b$ and $a+b=n$.

Proof. By Lemma 2.1.4, $\sum_{i=1}^{n} \lambda_{i}^{4}=2 M_{1}-2 e+8 f$. Thus $\lambda_{1}^{4}+\lambda_{n}^{4} \leq 2 M_{1}-2 e+8 f$ and $-\sqrt[4]{2 M_{1}-2 e+8 f-\lambda_{1}^{4}} \leq \lambda_{n} \leq \sqrt[4]{2 M_{1}-2 e+8 f-\lambda_{1}^{4}}$.

Therefore,

$$
S P(G)=\lambda_{1}-\lambda_{n} \leq \lambda_{1}+\sqrt[4]{2 M_{1}-2 e+8 f-\lambda_{1}^{4}}
$$

Equality holds if and only if $\lambda_{2}=\lambda_{3}=\cdots=\lambda_{n-1}=0$, that is, if and only if $A(G)=0$ or $\operatorname{rank}(A)=2$, equivalently, if and only if the non-isolated vertices of $G$ have at most two distinct neighborhood sets. Thus, equality holds if and only if $e=0$ or $G=K_{a, b}$ for some $a, b$ with $e=a b$ and $a+b=n$. If $e=a b$, then $\lambda_{1}=-\lambda_{n}=\sqrt{a b}$. Note that $\lambda_{1}+\sqrt[4]{2 M_{1}-2 e+8 f-\lambda_{1}^{4}}$ is a strictly increasing function of $\lambda_{1}$ when $\lambda_{1} \leq \sqrt[4]{M_{1}-e+4 f}$, and it is strictly decreasing when $\lambda_{1} \geq \sqrt[4]{M_{1}-e+4 f}$, we have

$$
S P(G) \leq \lambda_{1}+\sqrt[4]{2 M_{1}-2 e+8 f-\lambda_{1}^{4}} \leq 2 \sqrt[4]{M_{1}-e+4 f}
$$

All equalities hold when $\lambda_{1}=-\lambda_{n}=\sqrt{a b}$. 
The bound of Theorem 2.1.17 is better than Gregory's Theorem since for a $K_{4}$-free $(n, e)$ graph, by Lemma 2.1.14 and Corollary 2.1.16, we have $2 M_{1}-2 e+8 f-\lambda_{1}^{4}=$ $\sum_{i=1}^{n} \lambda_{i}^{4}-\lambda_{1}^{4} \leq 2 e^{2}-\lambda_{1}^{4} \leq 4 e^{2}-4 e \lambda_{1}^{2}+\lambda_{1}^{4}$. Thus $\sqrt[4]{2 M_{1}-2 e+8 f-\lambda_{1}^{4}} \leq \sqrt{2 e-\lambda_{1}^{2}}$ holds for any $K_{4}$-free $(n, e)$ graph.

Now we turn to the maximal Laplacian spread of a tree in Fan et al. [6]. The Laplacian matrix of a graph $G$ is defined as $L=L(G)=D(G)-A(G)$, where $D(G)=\operatorname{diag}\left\{d_{1}, \ldots, d_{n}\right\}$ is a diagonal matrix with the degree of a vertex $v_{i}$ of $G$ as the diagonal entries, $A(G)$ is the adjacency $n \times n$ matrix of $\mathrm{G}$ given by: $a_{i j}=1$ if vertices $v_{i}, v_{j}$ are adjacent and $a_{i j}=0$ otherwise. It is well known that $L$ is symmetric and positive semidefinite so the corresponding eigenvalues of $L$ can be arranged as follows:

$$
\lambda_{1}(G) \geq \lambda_{2}(G) \geq \cdots \geq \lambda_{n}(G)=0
$$

The Laplacian spread of the graph $G$ is then defined as

$$
S P(G)=\lambda_{1}(G)-\lambda_{n-1}(G)
$$

where $\lambda_{1}(G)$ is the spectral radius of $L$, and $\lambda_{n-1}(G)$ is called algebraic connectivity, which has a beautiful structural property given by Fiedler [7].

Theorem 2.1.18 ([6]) For $n \geq 5$, the star is the unique tree with maximal Laplacian spread among all trees of order $n$. 
Remark: By Theorem 2.1.18, the maximal Laplacian spread among all trees of order $n$ is $n-1$, and the related matrix $L$ must then be,

$$
\left(\begin{array}{cc}
n-1 & -e_{(n-1) \times 1}^{T} \\
-e_{(n-1) \times 1} & I_{n-1}
\end{array}\right)
$$




\subsection{Consequences, Facts and Other Results}

We will introduce an important result, Theorem 2.2 .3 , which provide an upper bound on the spread of a matrix, and describes when this bound is attained. Using this fact, we will focus on some certain normal matrices, and find the maximum spread for this class of normal matrices, and determine the corresponding structure of the matrices.

\subsubsection{Mirsky's Theorem}

Lemma 2.2.1 Let $z_{1}, \ldots, z_{n}$ be arbitrary complex numbers, $n \geq 3, t \geq 1$, and denote

$$
\begin{array}{r}
s=\max _{i, j}\left|z_{i}-z_{j}\right|, \\
M=\sum_{i=1}^{n}\left|z_{i}\right|^{t} .
\end{array}
$$

Then

$$
s \leq 2(M / 2)^{1 / t}
$$

with equality if and only if $z_{p}+z_{q}=0$ for some $p, q \in\{1, \ldots, n\}, p \neq q$, and $z_{k}=0$ for $k \neq p, q$.

Proof. From (2.19), we know $\left|z_{j}\right| \leq M^{1 / t}$. Assume $\left|z_{i}\right|=r M^{1 / t}$, where $r \in[0,1]$. Then for $j \neq i$ we have

$$
\left|z_{j}\right| \leq M^{1 / t}\left(1-r^{t}\right)^{1 / t}
$$

and consequently,

$$
\left|z_{i}-z_{j}\right| \leq\left|z_{i}\right|+\left|z_{j}\right| \leq M^{1 / t}\left[r+\left(1-r^{t}\right)^{1 / t}\right] \quad(i \neq j)
$$


The function: $r \rightarrow r+\left(1-r^{t}\right)^{1 / t}$ has a maximum equal to $2^{1-1 / t}$ when $r^{t}=\frac{1}{2}$. It follows that

$$
\left|z_{i}-z_{j}\right| \leq 2(M / 2)^{1 / t} \quad(i, j=1, \ldots, n)
$$

whence the inequality $(2.20)$ holds at once. One can easily see from (2.21) that in order to have equality in (2.22) one must have $\left|z_{i}\right|=\left|z_{j}\right|=(M / 2)^{1 / t}$ and $\arg z_{j}=\pi+\arg z_{i}$. These relations, together with the definition of $M$, imply that $z_{i}+z_{j}=0$ and $z_{k}=0$ for $k \neq i, j$. Clearly, these latter conditions are sufficient in order to have equality in (2.21) and therefore also in (2.20).

Let $\xi \in \mathbb{C}$, and $\lambda_{i}(\xi)$ be the $i^{\text {th }}$ element in $\sigma(A-\xi I)$, when the eigenvalues of $A-\xi I$ are ordered as $\left|\lambda_{1}(\xi)\right| \geq\left|\lambda_{2}(\xi)\right| \geq \cdots \geq\left|\lambda_{n}(\xi)\right|$; recall that $\Lambda(A)$ denotes the set of singular values of $A$, namely, the square roots of the eigenvalues of the nonnegative Hermitian matrix $A A^{*}$, and let $\Lambda_{i}(\xi)$ be the $i^{\text {th }}$ element in $\Lambda(A-\xi I)$. We have the following lemma.

Lemma 2.2.2 Let $A$ be a complex $n \times n$ matrix $(n \geq 3)$, let $\xi \in \mathbb{C}$, and let $t \geq 1$. Then

$$
S P(A) \leq 2 \sqrt[t]{\frac{1}{2} \sum_{i=1}^{n} \Lambda_{i}^{t}(\xi)} .
$$

Proof. Note that $S P(A)=S P(A-\xi I)$. By H.Weyl's Theorem [29], we have

$$
\sum_{i=1}^{n} \lambda_{i}^{t}(\xi) \leq \sum_{i=1}^{n} \Lambda_{i}^{t}(\xi) .
$$

Now, the statement of this lemma follows from an application of Lemma 2.2.1. 
We are now in a position to state and prove a very important result, due to Mirsky, on the spread of a general matrix. The proof technique is similar to that of Mirsky [19]. Recall that $\operatorname{tr}(A)$ is the trace of the matrix $A, \operatorname{tr}(A)=\sum_{i=1}^{n} a_{i i}, A=\left[a_{i j}\right] \in \mathbb{C}^{n \times n}$, and that $\|A\|_{F}$ denotes the Frobenious $N o r m$ of $A$, i.e. $\|A\|_{F}^{2}=\operatorname{tr}\left(A A^{*}\right)$, and when $A$ is normal, $\|A\|_{F}^{2}=\sum_{i, j \in\{1, \ldots, n\}} a_{i j}^{2}$.

Theorem 2.2.3 (Mirsky[19]) Let $A$ be a complex $n \times n$ matrix $(n \geq 3$.) Then

$$
S P(A) \leq \sqrt{2\|A\|_{F}^{2}-\frac{2}{n}|\operatorname{tr}(A)|^{2}} .
$$

with equality if and only if $A$ is normal and $n-2$ of its eigenvalues are equal to each other and this common value is the arithmetic mean of the remaining two.

Proof. We have

$$
\begin{aligned}
\sum_{i=1}^{n} \Lambda_{i}^{2}(\xi) & =\operatorname{tr}\left[(A-\xi I)^{*}(A-\xi I)\right] \\
& =\operatorname{tr}\left(A^{*} A-\bar{\xi} A-\xi A^{*}+|\xi|^{2} I\right) \\
& =\|A\|_{F}^{2}-\frac{1}{n}|\operatorname{tr}(A)|^{2}+n\left|\xi-\frac{1}{n} \operatorname{tr}(A)\right|^{2}(\xi \in \mathbb{C})
\end{aligned}
$$

Clearly, the choice $\xi=\frac{\operatorname{tr}(A)}{n}$ yields the smallest possible value for this last expression. Taking, in (2.23), $t=2$ and $\xi=\frac{\operatorname{tr}(A)}{n}$ we obtain the inequality (2.25). It is known that, in the case $t=2$, we have equality in (2.24) if and only if $A-\xi I$ is normal, i.e., if and only if $A$ is normal. This, together with the conditions for equality in (2.20), applied to the eigenvalues of $A-\xi I$, gives the conditions for equality in (2.25). 
Remark: Here we write the condition, if $n$ complex numbers are such that $n-2$ among them are equal to each other and this common value is the arithmetic mean of the remaining two, as condition $\mathscr{C}$.

When the characteristic roots of $A$ are all real, the following result will yield an upper bound generally better than that of Theorem 2.2.3.

Theorem 2.2.4 (Mirsky[19]) If all characteristic roots of the $n \times n$ matrix $A$ are real, then

$$
S P(A) \leq\left\{2\left(1-\frac{1}{n}\right)(\operatorname{tr}(A))^{2}-4 t r_{2} A\right\}^{1 / 2},
$$

where $\operatorname{tr}_{2} A$ is the sum of all $2 \times 2$ principal minors of $A$, with equality if and only if the characteristic roots satisfy $\mathscr{C}$.

Note: The reason why Mirsky's result is useful for this work is because: when the spread of $A$ attains the bound in (2.25), then $A$ is a normal matrix and has a very special property of the eigenvalues. Throughout my thesis, we want to find the maximum spread of an $n \times n$ normal matrix, in some sense, which has a connection to the condition $\mathscr{C}$, and the rank of $A$. 


\section{Chapter 3}

\section{Maximum Spread for Certain Classes of}

\section{Normal Matrices}

In this chapter, we focus on the maximum spread and the related structure of certain classes of normal matrices, including real symmetric matrices with entries in a specific interval, real skew symmetric matrices, complex Hermitian matrices with entries of modulus no more than 1, and skew Hermitian matrices with entries of modulus no more than 1. In this discussion, Theorem 2.2.3 (Mirsky's Theorem) plays an important role, as outlined in the note in the previous section.

\subsection{Case of Real Normal Matrices}

Let $J_{n}$ denote a matrix of order $n$, whose entries are all ones, let $N_{n}[a, b]$ denote the set of $n \times n$ normal matrices all of whose entries lie in the interval $[a, b]$, and let $S_{n}[a, b]$ denote the set of $n \times n$ real symmetric matrices, contained in $N_{n}[a, b]$. Let $\mathscr{U}_{n}$ denote the set of unitary matrices, i.e, for any matrix $U \in \mathscr{U}_{n}, U U^{*}=U^{*} U=I$. 
For an $n \times n$ real symmetric matrix $A$, we always denote the eigenvalues of $A$ in decreasing order, $\lambda_{1}(A) \geq \cdots \geq \lambda_{n}(A)$.

Recall from Chapter 2 that the spread of an $n \times n$ matrix $A$ is defined as

$$
S P(A)=\max _{i, j=1, \ldots, n}\left|\lambda_{i}(A)-\lambda_{j}(A)\right|
$$

This quantity has applications in certain combinatorial optimization problems [8]. In this chapter we investigate the maximum value of $S P(A)$ for $A \in N_{n}[a, b]$.

Lemma 3.1.1 ([11]) Let $A \in M_{n}(\mathbb{R})$, then $A$ is normal if and only if there exists a real orthogonal matrix $Q \in M_{n}(\mathbb{R})$, such that

$$
Q^{T} A Q=\left(\begin{array}{cccc}
A_{1} & & & \\
& & & 0 \\
& A_{2} & & \\
& & \ddots & \\
& & & A_{k}
\end{array}\right) \in M_{n}(\mathbb{R}), 1 \leq k \leq n,
$$

where $A_{i}$ is either a real 1-by-1 matrix or is a real 2-by-2 matrix of the form

$$
A_{i}=\left(\begin{array}{cc}
a_{i} & b_{i} \\
-b_{i} & a_{i}
\end{array}\right)
$$

We also write $Q=\left(q_{1}, q_{2}, \ldots, q_{n}\right)$, where $q_{i}=\left(q_{i_{1}}, q_{i_{2}}, \ldots, q_{i_{n}}\right)^{T}$ is the $i^{\text {th }}$ column of $Q$.

Corollary 3.1.2 $A \in M_{n}(\mathbb{R})$ is a normal matrix. Then $A$ is symmetric if and only if $\sigma(A) \subseteq \mathbb{R}$. 
Lemma 3.1.3 Suppose $A \in M_{n}(\mathbb{R})$ is a normal matrix. If $S P(A)$ attains the upper bound from Theorem 2.2.3, then either all the eigenvalues are real or there is exactly one pair of complex conjugate eigenvaleues. In the latter case, the remaining $n-2$ real eigenvalues are equal to the real part of the complex eigenvalues.

Proof. By Theorem 2.2.3, if $S P(A)=\sqrt{2\|A\|_{F}{ }^{2}-\frac{2}{n}|\operatorname{tr}(A)|^{2}}$, then $A$ is a normal matrix and $n-2$ of its eigenvalues are all equal to the arithmetic mean of the remaining two. Since $S P(A)=\max _{\lambda_{i}, \lambda_{j} \in \sigma(A)}\left|\lambda_{i}-\lambda_{j}\right|$, we consider different cases for calculating the value of this spread.

Case 1: $\sigma(A) \subseteq \mathbb{R}$. Then $S P(A)=\lambda_{1}-\lambda_{n}$ and all the other eigenvalues are equal to $\frac{\lambda_{1}+\lambda_{n}}{2}$. By Lemma 3.1.1, $A$ is a real symmetric matrix and the rank of $A$ is either $0,2, n-1$, or $n$.

Case 2: Suppose $A$ has complex eigenvalues, which must occur in conjugate pairs. Assume $0<S P(A)=\left|\lambda_{i}-\lambda_{j}\right|$ for some pair of eigenvalues $\lambda_{i}, \lambda_{j}$.

Subcase 2.1: If $\lambda_{i}, \lambda_{j} \in \mathbb{R}$, then $\sigma(A) \subseteq \mathbb{R}$ and Case 1 applies.

Subcase 2.2: If $\lambda_{i} \in \mathbb{R}$, and $\lambda_{j}=a+i b \in \mathbb{C}(b \neq 0)$, then the other eigenvalues are equal to $\frac{\lambda_{1}+a}{2}+i \frac{b}{2}$ which forces $\frac{b}{2}=-b$, and then we reach a contradiction.

Subcase 2.3: If $\lambda_{i}=c+i b=\bar{\lambda}_{j}$, then the remaining eigenvalues are equal to $c \in \mathbb{R}$. In this case, the rank of $A$ is either 2 or $n$.

Subcase 2.4: If $\lambda_{i}=c_{1}+i b_{1}, \lambda_{j}=c_{2}+i b_{2} \in \mathbb{C}$, but not conjugate, then the 
other eigenvalues are all equal to $\frac{c_{1}+c_{2}}{2}+i \frac{b_{1}+b_{2}}{2}$, and since conjugate eigenvalues must occur in pairs, we reach a contradiction. This completes the proof.

Remark (*): If matrix $A$ satisfies the conditions in Lemma 3.1 .3 , then the possible ranks of $A$ must be one of the following: if $S P(A)=\left|\lambda_{i}-\lambda_{j}\right|$ for some $i, j$ :

- $\lambda_{i}, \lambda_{j} \neq 0, \lambda_{i}+\lambda_{j} \neq 0 \Rightarrow \operatorname{rank}(A)=n$

- $\lambda_{i}=0, \lambda_{j} \neq 0, \lambda_{i}+\lambda_{j} \neq 0$ or $\lambda_{i} \neq 0, \lambda_{j}=0, \lambda_{i}+\lambda_{j} \neq 0 \Rightarrow \operatorname{rank}(A)=n-1$

- $\lambda_{i}, \lambda_{j} \neq 0, \lambda_{i}+\lambda_{j}=0 \Rightarrow \operatorname{rank}(A)=2$

- $\lambda_{i}=0, \lambda_{j}=0, \lambda_{i}+\lambda_{j}=0 \Rightarrow \operatorname{rank}(A)=0$ 


\section{Remarks:}

1. In Case 1 above, if $S P(A)$ is maximum and $A \in S_{n}[a, 1]$, then, as $A$ is symmetric,

it follows that the entries of $A$ are either $a$ or 1 (see Lemma 3.2.1).

2. In Case 3 , let

$$
\begin{aligned}
U & =Q\left(\begin{array}{cc:c}
\frac{1}{\sqrt{2}} & \frac{1}{\sqrt{2}} & O_{2 \times(n-2)} \\
\frac{i}{\sqrt{2}} & \frac{-i}{\sqrt{2}} & \ldots \\
\hdashline O_{(n-2) \times 2} & I_{(n-2) \times(n-2)}
\end{array}\right) \in \mathscr{U}_{n} \\
& =\left(\begin{array}{cccc}
\frac{q_{1}+i q_{2}}{\sqrt{2}}, & \frac{q_{2}-i q_{2}}{\sqrt{2}}, & q_{3}, \ldots, & q_{n}
\end{array}\right) .
\end{aligned}
$$

Then

$$
U^{*} A U=\left(\begin{array}{cr:c}
c+i b & 0 & O_{2 \times(n-2)} \\
0 & c-i b & \\
\hdashline O_{(n-2) \times 2} & c I_{(n-2) \times(n-2)}
\end{array}\right)
$$

and

$$
Q^{T} A Q=\left(\begin{array}{cc:c}
c & b & 0_{2 \times(n-2)} \\
-b & c & \\
\hdashline O_{(n-2) \times 2} & c I_{(n-2) \times(n-2)}
\end{array}\right)
$$

Now $B=A-c I=b\left(q_{1} q_{2}^{T}-q_{2} q_{1}^{T}\right)$ is a real skew symmetric matrix, which is rank 2, where $q_{1}, q_{2}$ are the associated eigenvectors, $q_{1}^{T} q_{1}=q_{2}^{T} q_{2}=1$, and $q_{1}^{T} q_{2}=q_{2}^{T} q_{1}=0$. Let $C=\left[c_{i j}\right]_{n \times n}=q_{1} q_{2}^{T}-q_{2} q_{1}^{T}$. Then $\|C\|_{F}^{2}=2$ and $\operatorname{tr}(C)=0$ and $0 \leq\left|c_{i j}\right|=$ $\left|\left(q_{1}\right)_{i}\left(q_{2}\right)_{j}-\left(q_{2}\right)_{i}\left(q_{1}\right)_{j}\right| \leq 1$, for $i, j=1, \ldots, n$. So $B=A-c I=b C$, the entries of $B$ are bounded in $[-b, b], b>0$, where $b$ is the imaginary part of the complex eigenvalues of $A$, and $c$ is the real part of those complex eigenvalues of $A$. Then the diagonal 
entries of $A$ are all $c$ and the off-diagonal entries of $A$ lie in $[-b, b]$.

We study these two cases, Case 1 and Subcase 2.3, in the next two sections. 


\subsection{Real Symmetric Matrices}

In this section, we focus on the class of real symmetric matrices, $S_{n}[a, b]$. We study the maximum spread of these real symmetric matrices and their related matrix structure.

\subsubsection{Structure of Real Symmetric Matrices Whose Spreads Attain the Bound from Mirsky's Theorem}

Lemma 3.2.1 Suppose $A \in S_{n}[a, b]$. If $S P(A)$ is maximum, then each entry of $A$ is either $a$ or $b$.

Proof. Throughout this proof we write $\lambda_{i}$ for $\lambda_{i}(A)$. For $x \in \mathbb{R}^{n}$, we always write its components as $x_{1}, \ldots, x_{n}$. Given $A \in S_{n}[a, b]$, let $x, y \in \mathbb{R}^{n}$ be unit eigenvectors such that $\lambda_{1}=x^{T} A x, \lambda_{n}=y^{T} A y$. Then

$$
S P(A)=x^{T} A x-y^{T} A y=e^{T}\left[A \circ\left(x x^{T}-y y^{T}\right)\right] e, \quad e=(1, \ldots, 1)^{T}
$$

Note that the $(i, j)$ entry of $x x^{T}-y y^{T}$ is $x_{i} x_{j}-y_{i} y_{j}$. We define a new matrix $\tilde{A}=\left(\tilde{a}_{i j}\right)$ as follows: $\tilde{a}_{i j}=b$ if $x_{i} x_{j}-y_{i} y_{j} \geq 0$ and $\tilde{a}_{i j}=a$ if $x_{i} x_{j}-y_{i} y_{j}<0$. Then from (3.26) we have

$$
\begin{aligned}
S P(A) & \leq x^{T} \tilde{A} x-y^{T} \tilde{A} y \\
& \leq \max \left\{\omega^{T} \tilde{A} \omega:\|\omega\|=1, \omega \in \mathbb{R}^{n}\right\}-\min \left\{z^{T} \tilde{A} z:\|z\|=1, z \in \mathbb{R}^{n}\right\} \\
& =\operatorname{SP}(\tilde{A}) .
\end{aligned}
$$


We will show that when $S P(A)$ is maximum there is no position $(i, j)$ in the related matrix such that $x_{i} x_{j}-y_{i} y_{j}=0$ (see Theorem 3.4.2 below). Therefore the maximum spread for a matrix in $S_{n}[a, b]$ can only be attained at some $\{a, b\}$-matrix (that is, a matrix in $S_{n}[a, b]$ whose entries are either equal to $a$ or $b$ ).

As a consequence of Lemma 3.2.1, we need to determine the structure for a matrix $A$ in $S_{n}-\{a, b\}$ (symmetric $n \times n$ matrices whose entries are either $a$ or $b$ ) when $a^{2} \leq b^{2}$, since $S P(A)=S P(-A)$. Furthermore by considering $b^{-1} A$ instead of $A$, we only study the maximum spread for matrices in $S_{n}-\{a, 1\} \quad(-1 \leq a<1)$.

We now focus on rank 2 matrices $A \in S_{n}-\{a, 1\} \quad(-1 \leq a<1)$. In this case, we can find an invertible principal submatrix of order 2. Assume, by simultaneous permutation of rows and columns of $A$, that this $2 \times 2$ submatrix lies in rows/columns 1,2. Thus every other row in this matrix is a linear combination of these two rows. From which we determine the structure of these matrices. Examples for $n=2$ and 
$n=3$ are given below (each with all real eigenvalues):

$$
\begin{gathered}
A_{2}^{1}=\left(\begin{array}{ll}
a & 1 \\
1 & 1
\end{array}\right) \quad A_{2}^{2}=\left(\begin{array}{ll}
a & a \\
a & 1
\end{array}\right) \quad A_{3}^{1}=\left(\begin{array}{lll}
a & 1 & 1 \\
1 & 1 & 1 \\
1 & 1 & 1
\end{array}\right) \\
A_{3}^{2}=\left(\begin{array}{lll}
a & 1 & a \\
1 & 1 & 1 \\
a & 1 & a
\end{array}\right) \quad A_{3}^{3}=\left(\begin{array}{ccc}
a & a & a \\
a & a & a \\
a & 1 & a \\
a & 1
\end{array}\right) \quad A_{3}^{5}=\left(\begin{array}{cc}
a & a
\end{array}\right) \\
A_{3}^{4}=\left(\begin{array}{ccc}
-\frac{1}{2} & 1 & -\frac{1}{2} \\
1 & -\frac{1}{2} & -\frac{1}{2} \\
-\frac{1}{2} & -\frac{1}{2} & 1
\end{array}\right) .
\end{gathered}
$$

We will show that $S P\left(A_{2}^{1}\right)$ is maximum when $n=2$ and $S P\left(A_{3}^{1}\right)$ is maximum when $n=3$.

From Chapter 2, we know that a symmetric real matrix order $n(n \geq 3)$, named a Mirsky matrix, will attain the largest possible spread (see Theorem 2.2.3) when $n-2$ eigenvalues coincide and are all equal to the arithmetic mean of the remaining two eigenvalues.

We now focus on the structure of a Mirsky matrix $A$, which lies in the class $S_{n}-\{a, 1\}$, with $a \in[-1,1)$. Take

$$
\xi=\frac{\operatorname{tr}(A)}{n}=\frac{\lambda_{1}(A)+\lambda_{n}(A)}{2}
$$


and let

$$
A^{\prime}=A-\xi I \text {. }
$$

Then, using Remark (*), we have

$$
S P\left(A^{\prime}\right)=S P(A), \quad \operatorname{tr}\left(A^{\prime}\right)=0, \quad \operatorname{rank}\left(A^{\prime}\right)=2 .
$$

We call $A^{\prime}$ the transform of $A$. In the following, we explore the structure of $A^{\prime}$.

Suppose that there are $s a^{\prime} s$ and $(n-s) 1^{\prime} s$ on the main diagonal of $A$ and observe $\operatorname{tr}(A)=a s+n-s$, with $0 \leq s \leq n$. Let

$$
\begin{aligned}
x & =\frac{1}{n}(a-1)(n-s) \in[-2,0], \\
y & =\frac{s}{n}(1-a) \in[0,2], \\
y-x & =1-a>0 .
\end{aligned}
$$

So there are $s x^{\prime} s,(n-s) y^{\prime} s$ on the main diagonal of $A^{\prime}$ while any off-diagonal entry is either $a$ or 1 in matrix $A^{\prime}$. Since $\operatorname{rank}\left(A^{\prime}\right)=2$, by simultaneous row-column permutation, we may assume that the upper left $2 \times 2$ principal submatrix of $A^{\prime}$ is nonsingular and therefore must be one of the following 6 cases:

$$
\begin{array}{ccc}
\left(\begin{array}{ll}
x & 1 \\
1 & x
\end{array}\right) & \left(\begin{array}{ll}
x & a \\
a & x
\end{array}\right) & \left(\begin{array}{ll}
y & 1 \\
1 & y
\end{array}\right) \\
\left.\begin{array}{cc}
\text { Case } 1 \\
y & a \\
a & y
\end{array}\right) & \left(\begin{array}{ll}
x & 1 \\
1 & y
\end{array}\right) & \text { Case } 2 \\
\text { Case } 4 & \text { Case } 5 & \left(\begin{array}{cc}
x & a \\
a & y
\end{array}\right)
\end{array}
$$


Set $\alpha, \beta$ as the first and the second row vector of $A^{\prime}$ respectively, and let $\gamma_{i}$ be a remaining arbitrary row vector. Then $\gamma_{i}=u_{i} \alpha+v_{i} \beta, u_{i}, v_{i} \in \mathbb{R}$ for $i=3, \ldots, n$, where $\left(u_{i}, v_{i}\right)$ are real numbers. We call this pair the combination parameters of row $i$.

\section{Remarks:}

1. $x \neq-1$ in Case $1 ;|x| \neq|a|$ in Case $2 ; y \neq 1$ in Case $3 ; y \neq|a|$ in Case 4; neither $x$ nor $y$ is 0 in Case 5 and Case 6.

2. When $n=2$, there are 4 types of Mirsky matrices $A^{\prime}$,

$$
\left(\begin{array}{ll}
0 & 1 \\
1 & 0
\end{array}\right)\left(\begin{array}{ll}
0 & a \\
a & 0
\end{array}\right)\left(\begin{array}{rr}
x & 1 \\
1 & -x
\end{array}\right)\left(\begin{array}{rr}
x & a \\
a & -x
\end{array}\right) \text {, }
$$

where $x=\frac{a-1}{2} \in[-1,0)$.

Consequently, the general structure of such a Mirsky matrix order $n$ is the following:

$\left(\begin{array}{cc|c|c|c|c}m_{1} & p & a J_{1 \times s_{1}} & a J_{1 \times s_{2}} & J_{1 \times s_{3}} & J_{1 \times s_{4}} \\ p & m_{2} & a J_{1 \times s_{1}} & J_{1 \times s_{2}} & a J_{1 \times s_{3}} & J_{1 \times s_{4}} \\ \hline a J_{s_{1} \times 1} & a J_{s_{1} \times 1} & x_{1} J_{s_{1} \times s_{1}} & y_{1} J_{s_{1} \times s_{2}} & y_{2} J_{s_{1} \times s_{3}} & y_{3} J_{s_{1} \times s_{4}} \\ \hline a J_{s_{2} \times 1} & J_{s_{2} \times 1} & y_{1} J_{s_{2} \times s_{1}} & x_{2} J_{s_{2} \times s_{2}} & y_{4} J_{s_{2} \times s_{3}} & y_{5} J_{s_{2} \times s_{4}} \\ \hline J_{s_{3} \times 1} & a J_{s_{3} \times 1} & y_{2} J_{s_{3} \times s_{1}} & y_{4} J_{s_{3} \times s_{2}} & x_{3} J_{s_{3} \times s_{3}} & y_{6} J_{s_{3} \times s_{4}} \\ \hline J_{s_{4} \times 1} & J_{s_{4} \times 1} & y_{3} J_{s_{4} \times s_{1}} & y_{5} J_{s_{4} \times s_{2}} & y_{6} J_{s_{4} \times s_{3}} & x_{4} J_{s_{4} \times s_{4}}\end{array}\right)$


where $s_{1}, s_{2}, s_{3}, s_{4} \in[0, n-2], s_{1}+s_{2}+s_{3}+s_{4}=n-2, n \geq 3, m_{1}, m_{2} \in\{x, y\}$, and $p \in\{a, 1\}$. When $s_{i} \geq 2, x_{i} \in\{1, a\} ; y_{j} \in\{1, a\}, i=1, \ldots, 4, j=1, \ldots, 6$.

Following is our main observation regarding the possible structure of a Mirsky matrix.

Theorem 3.2.2 If $A$ is a Mirsky matrix in $S_{n}-\{a, 1\}, a \in[-1,1), n \geq 3$, then $A^{\prime}$, the transform of $A$, must be in one of the following ten forms:

$$
\begin{aligned}
& \begin{array}{l}
\left(\begin{array}{rrr}
-\frac{1}{2} J_{s_{1}} & J_{s_{1} \times s_{2}} & -\frac{1}{2} J_{s_{1} \times s_{3}} \\
J_{s_{2} \times s_{1}} & -\frac{1}{2} J_{s_{2}} & -\frac{1}{2} J_{s_{2} \times s_{3}} \\
-\frac{1}{2} J_{s_{3} \times s_{1}} & -\frac{1}{2} J_{s_{3} \times s_{2}} & J_{s_{3}}
\end{array}\right), \quad\left(\begin{array}{rrr}
2 a-1 & 1 & a \\
1 & (2 a-1) & a J_{1 \times(n-2)} \\
a & a J_{(n-2) \times 1} & a J_{n-2}
\end{array}\right), \\
1 \leq s_{1}, s_{2} \leq n-2, s_{3}=\frac{1}{2}\left(s_{1}+s_{2}\right)
\end{array} \\
& \left(\begin{array}{rr}
a J_{s} & a J_{s \times(n-s)} \\
a J_{(n-s) \times s} & J_{n-s}
\end{array}\right), \quad\left(\begin{array}{rr}
a J_{s} & J_{s \times(n-s)} \\
J_{(n-s) \times s} & J_{n-s}
\end{array}\right), \\
& a=\frac{s-n}{s}, s=1: n-1 \quad a=\frac{s-n}{s}, s=1: n-1 \\
& \left(\begin{array}{rr}
a J_{n-1} & a J_{(n-1) \times 1} \\
a J_{1 \times(n-1)} & 2 a-1
\end{array}\right), \quad\left(\begin{array}{cc}
a J_{n-1} & J_{(n-1) \times 1} \\
J_{1 \times(n-1)} & 2 a-1
\end{array}\right), \\
& a=\frac{1}{1+n} \quad a=\frac{1}{1+n}
\end{aligned}
$$




$$
\left(\begin{array}{ccc}
0_{s_{1}} & J_{s_{1} \times s_{2}} & 0_{s_{1} \times s_{3}} \\
J_{s_{2} \times s_{1}} & 0_{s_{2}} & 0_{s_{2} \times s_{3}} \\
0_{s_{3} \times s_{1}} & 0_{s_{3} \times s_{2}} & 0_{s_{3}}
\end{array}\right)
$$

where $1 \leq s_{1}, s_{2} \leq n-1, s_{1}+s_{2}+s_{3}=n$,

$$
\left(\begin{array}{rrr}
\frac{a-1}{3} & 1 & a \\
1 & \frac{a-1}{3} & 1 \\
a & 1 & \frac{2(1-a)}{3}
\end{array}\right)
$$

where $a$ is the unique solution of $11 a^{3}-15 a^{2}-57 a+7=0$, with $a \in[-1,1)$,

$$
\left(\begin{array}{rrr}
\frac{1-a}{3} & 1 & a \\
1 & \frac{1-a}{3} & 1 \\
a & 1 & \frac{2(a-1)}{3}
\end{array}\right)
$$

where $a$ is the unique solution of $11 a^{3}-15 a^{2}+51 a+7=0$, with $a \in[-1,1)$,

$$
\left(\begin{array}{rrr}
\frac{1-a}{3} & a & a \\
a & \frac{1-a}{3} & 1 \\
a & 1 & \frac{2(a-1)}{3}
\end{array}\right),
$$

where $a$ is one of the two solutions of $7 a^{3}-57 a^{2}-15 a+11=0$, with $a \in[-1,1)$. 
Proof. The order 2 nonsingular principal matrix is in one of the 6 cases showed in (3.30). We then determine the structure of a Mirsky matrix $A^{\prime}$ as the other rows must be linear combinations of these first two rows, we include the proof here for Case 1 , the remaining analysis may be performed in a similar manner for the other cases.

Let $B_{1}^{\prime}, B_{2}^{\prime}$ be the leading order 3 and order 4 submatrices, respectively, that contain the order 2 principal matrix as in Case 1 in (3.30). We will determine the structure of a Mirsky matrix $A^{\prime}$ in two steps.

Step 1: Study the structure of the Mirsky matrix with submatrix $B_{1}^{\prime}$, which has the same remaining row vectors starting from the $3^{\text {rd }}$ row, and let $s_{i}, i=1, \ldots, 4$, be the indexes of the respective blocks in matrix $(3.31)$ and $(u, v)$ the combination parameters for the remaining rows in $A^{\prime}$. We begin with case analysis starting with Case 1 from (3.30). This analysis will be divided into four subcases as follows:

Case 1.1.1:

$$
\left(\begin{array}{ll|l}
x & 1 & a \\
1 & x & a \\
\hline a & a & x
\end{array}\right) v
$$

It follows that

$$
\left\{\begin{array}{l}
u x+v=a \\
u+v x=a
\end{array} \Rightarrow(u-v)(x-1)=0, u=v \text { since } x<0, \text { so } 2 u a=x, u(1+x)=a\right. \text {. }
$$


If $u=0$, then $a=0, x=0$ and $s=n$; else $u \neq 0$, then $a \neq 0, x^{2}+x-2 a^{2}=0$. If $s_{1} \geq 2$, then $x=a<0$, which implies $a^{2}+a-2 a^{2}=0$, a contradiction, so $s_{1} \leq 1$, and when $n=3, s=3$, then $x=0=a, u=v=0$, a contradiction.

Case 1.1.2:

$$
\left(\begin{array}{ll|l}
x & 1 & a \\
1 & x & a \\
\hline a & a & y
\end{array}\right) v
$$

By Case 1.1.1, $u=v$, so $2 u a=y, u(1+x)=a$. If $u=0$, then $y=0=a, s=0$, a contradiction; so $u \neq 0$, and it follows that $a \neq 0,(1+x) y=(1+x)(1+x-a)=2 a^{2}$. Then

$$
\begin{aligned}
& x=2 a-1, y=a, u=v=\frac{1}{2}, s=\frac{n a}{1-a}, a \in\left(0, \frac{1}{2}\right) \\
& \text { or } x=-1-a, y=-2 a, u=v=-1, s=-\frac{2 n a}{1-a}, a \in(-1,0) .
\end{aligned}
$$

If $s_{1}=n-2$, it follows that $y=a, x=2 a-1, a=\frac{2}{n+2}$, or $y=-2 a, x=-1-a$, and $a=\frac{1}{1-n}$.

Case 1.2.1:

$$
\left(\begin{array}{ll|l}
x & 1 & a \\
1 & x & 1 \\
\hline a & 1 & x
\end{array}\right) v
$$


It follows that

$$
\left\{\begin{array}{l}
u x+v=a \\
u+v x=1
\end{array} \Rightarrow(u-v)(x-1)=a-1 \text { and }(u+v)(x+1)=a+1\right.
$$

If $a=-1$, then $u=-v$, so $a u+v=2 v=x, 2 v(x-1)=2$, which means $x^{2}-x-2=0, x=-1$, a contradiction. So $a \neq-1$, and $u=\frac{a x-1}{x^{2}-1}, v=\frac{x-a}{x^{2}-1}$. Then we have $(x-a)\left(x^{2}+a x-2\right)=0$ by $a u+v=x$. If $s_{2}=n-2$, then it follows that $x=a=0$.

Case 1.2.2:

$$
\left(\begin{array}{ll|l}
x & 1 & a \\
1 & x & 1 \\
\hline a & 1 & y
\end{array}\right) v
$$

By Case 1.2.1, $a \neq-1$ otherwise $u=-v, a u+v=2 v=y, 2 v(x-1)=2$, which means $y(x-1)=2$, obviously a contradiction. So we have

$$
x^{3}+(1-a) x^{2}-\left(a^{2}+2\right) x+(3 a-1)=0 .
$$

If $s_{2} \geq 2$, then $y=1, x=a<0$, it follows that $a^{2}-1=\left(a^{2}+1\right) a-2 a, a=-1$, a contradiction; or $y=a>0, x=2 a-1<0$. Hence $2 a^{3}-3 a^{2}+1=0$, but there is no root in the interval $\left(0, \frac{1}{2}\right)$, so $s_{2} \leq 1$. When $n=3$, it follows $x=\frac{a-1}{3}, y=\frac{2}{3}(1-a), 11 a^{3}-15 a^{2}-57 a+7=0$ and there is a unique solution $a$ in the interval $(-1,1)$. 
By symmetry, similar results hold in Case 1.3 as in Case 1.2 .

Remark: Case 1.1, Case 1.2, Case 1.3, and Case 1.4 mean the $3^{\text {rd }}$ row vector in $A^{\prime}$ follows as $(a, a, \ldots),(a, 1, \ldots),(1, a, \ldots)$, and $(1,1, \ldots)$, respectively. Case 1.1 .1 and Case 1.3.2 mean the $3^{\text {rd }}$ row vectors follow as $(a, a, x, \ldots),(1, a, y, \ldots)$. The similar idea is applied to the other cases.

Case 1.4.1:

$$
\left(\begin{array}{ll|l}
x & 1 & 1 \\
1 & x & 1 \\
\hline 1 & 1 & x
\end{array}\right) u
$$

It follows that

$$
\left\{\begin{array}{l}
u x+v=1 \\
u+v x=1
\end{array} \Rightarrow(u-v)(x-1)=0, u=v \text { since } x \leq 0 .\right.
$$

So $2 u=x, 2 u(1+x)=2, u \neq 0$ then $x^{2}+x-2=0, x=-2$, a contradiction since it implies $s=0$. So Case 1.4.1 is impossible. 
Case 1.4.2:

$$
\left(\begin{array}{ll|l}
x & 1 & 1 \\
1 & x & 1 \\
\hline 1 & 1 & y
\end{array}\right) v
$$

By Case 1.4.1, $u=v$, so $2 u=y, y(1+x)=2$. If $s_{4} \geq 2$, then it follows that $y=a>$ $0,1+x=2 a, 2 a^{2}=2$, a contradiction; or $y=1, x=a<0, y(1+x)=1+a=2$, also a contradiction. So $s_{4} \leq 1$, when $n=3$, it follows $y=\frac{2}{3}(1-a), x=\frac{a-1}{3}, a^{2}+a+7=0$, there is no real solution.

Step 2: Now, we study the cases for which there are all possible combinations with the remaining row vectors in a Mirsky matrix $A^{\prime}$ with submatrix $B_{2}^{\prime}$.

If the main diagonal of $A^{\prime}$ consists of all $x^{\prime} s$, it follows that $x=a=0$. Then it follows that the structure of the Mirsky matrix, after simultaneous row and column permutations, in Case 1 is as follows:

$$
\left(\begin{array}{lll}
0_{s_{2}+1} & J_{\left(s_{2}+1\right) \times\left(s_{3}+1\right)} & 0_{\left(s_{2}+1\right) \times s_{1}} \\
J_{\left(s_{3}+1\right) \times\left(s_{2}+1\right)} & 0_{s_{3}+1} & 0_{\left(s_{3}+1\right) \times s_{1}} \\
0_{s_{1} \times\left(s_{2}+1\right)} & 0_{s_{1} \times\left(s_{3}+1\right)} & 0_{s_{1}}
\end{array}\right),
$$

where $0 \leq s_{i} \leq n-2, \sum_{i} s_{i}=n-2,1 \leq i \leq 3$. 
In the arguments below we combine the previous cases depending on the possible $3^{\text {rd }}$ and $4^{\text {th }}$ rows. For example, Case 1.1.1-1.2.1 means the $3^{\text {rd }}$ row follows Case 1.1.1 and the $4^{\text {th }}$ row follows Case 1.2.1. When $x \neq 0$, we study the possibility of a combination with different $3^{\text {rd }}$ and $4^{\text {th }}$ row vectors with the combination parameters $\left(u_{1}, v_{1}\right),\left(u_{2}, v_{2}\right)$, respectively. From the discussion above, we have the following possibilities.

Case 1.1.1-1.2.1:

$$
\left(\begin{array}{ll|ll}
x & 1 & a & a \\
1 & x & a & 1 \\
\hline a & a & x & \star \\
a & 1 & \star & x
\end{array}\right) .
$$

From Case 1.1.1 and 1.2.1, we have $a \neq 0, x^{2}+x-2 a^{2}=0 ;$ and $a \neq-1$,

$$
(x-a)\left(x^{2}+a x-2\right)=0 .
$$

So $x=-2(1+a), a=\frac{\sqrt{5}-3}{2}$, then $\sqrt{5}(n-s)=2 n$, no such real numbers $n$ and $s$ exist. So this combination is impossible. By symmetry, the combination of Case 1.1.1-1.3.1 is also impossible. 
Case 1.1.1-1.2.2:

$$
\left(\begin{array}{ll|ll}
x & 1 & a & a \\
1 & x & a & 1 \\
\hline a & a & x & \star \\
a & 1 & \star & y
\end{array}\right)\left(\begin{array}{ll}
\left(u_{1}, v_{1}\right) \\
\left(u_{2}, v_{2}\right)
\end{array} .\right.
$$

By the results in Case 1.1.1 and 1.2.2, we have $a \neq 0, x^{2}+x-2 a^{2}=0$; and $a \neq-1, x^{3}+(1-a) x^{2}-\left(a^{2}+2\right) x+3 a-1=0$, which follows $(a+2) x=2 a^{2}+2 a-1$. Since $u_{1}(x+1)=a$, then $u_{1}(a+1)=\frac{a(a+1)}{x+1}=a$ or 1 , which implies $x=a<0$ or $x=a^{2}+a-1$, thus $a-a^{2}=0$ or $(a+2)\left(a^{2}+a-1\right)=2 a^{2}+2 a-1$, it follows $a=0, a=1$, or $a=-1$ which is a contradiction. So this combination is impossible. By symmetry, Case 1.1.1-1.3.2 is also impossible.

\section{Case 1.1.1-1.4.2:}

$$
\left(\begin{array}{cc|cc}
x & 1 & a & 1 \\
1 & x & a & 1 \\
\hline a & a & x & \star \\
1 & 1 & \star & y
\end{array}\right)\left(\begin{array}{l}
\left(u_{1}, v_{1}\right) \\
\left(u_{2}, v_{2}\right)
\end{array}\right.
$$

By the results in Case 1.1.1 and 1.4.2, we have $a \neq 0, x^{2}+x-2 a^{2}=0$, and $x^{2}+(2-a) x-(a+1)=0$, it implies that $x=2 a+1$ and $a=\frac{\sqrt{5}-3}{2}$. Since $s_{1}=s_{4}=1, x=\frac{a-1}{4}, y=\frac{3}{4}(1-a)$, we have $a=-\frac{5}{7}$, a contradiction. So this combination is impossible. 
Case 1.1.2-1.2.1:

$$
\left(\begin{array}{cc|cc}
x & 1 & a & a \\
1 & x & a & 1 \\
\hline a & a & y & \star \\
a & 1 & \star & x
\end{array}\right)\left(\begin{array}{ll}
\left(u_{1}, v_{1}\right) \\
\left(u_{2}, v_{2}\right)
\end{array} .\right.
$$

By the results in Case 1.1.2 and 1.2.1, we have $x=2 a-1, y=a, a \in\left(0, \frac{1}{2}\right)$ or $x=-1-a<0, y=-2 a>0 ; a \neq-1,(x-a)\left(x^{2}+a x-2\right)=0$. When $x=a$, we have $a=2 a-1, a=1$, a contradiction or $a=-a-1$, then $x=a=-\frac{1}{2}, y=1, s=\frac{2 n}{3}$; when $x^{2}+a x-2=0$, if $x=2 a-1$, it follows $a=1$ or $a=-\frac{1}{6}$, a contradiction; if $x=-1-a$, it follows $a=1$, a contradiction. We know the combination is possible for Case 1.1.2 and 1.2.1 when $x=a=-\frac{1}{2}, y=1, s=\frac{2 n}{3}$. By symmetry, there is a combination of Case 1.1.2-1.2.1-1.3.1. By simultaneous row and column permutations, we get the Mirsky matrix of the form

$$
\left(\begin{array}{lll}
J_{s_{1}} & -\frac{1}{2} J_{s_{1} \times\left(s_{2}+1\right)} & -\frac{1}{2} J_{s_{1} \times\left(s_{3}+1\right)} \\
-\frac{1}{2} J_{\left(s_{2}+1\right) \times s_{1}} & -\frac{1}{2} J_{s_{2}+1} & J_{\left(s_{2}+1\right) \times\left(s_{3}+1\right)} \\
-\frac{1}{2} J_{\left(s_{3}+1\right) \times s_{1}} & J_{\left(s_{3}+1\right) \times\left(s_{2}+1\right)} & -\frac{1}{2} J_{s_{3}+1}
\end{array}\right),
$$

where $0 \leq s_{i} \leq n-3, i=2$ or $3, s_{1}=\frac{n}{3}$. 
Case 1.1.2-1.2.2:

$$
\left(\begin{array}{cc|cc}
x & 1 & a & a \\
1 & x & a & 1 \\
\hline a & a & y & \star \\
a & 1 & \star & y
\end{array}\right)\left(\begin{array}{ll}
\left(u_{1},\right. & \left.v_{1}\right) \\
\left(u_{2},\right. & \left.v_{2}\right)
\end{array} .\right.
$$

By the results in Case 1.1.2 and 1.2.2, we have $a \neq 0, x=2 a-1, y=a$ or $x=-1-a, y=-2 a$, and $a \neq-1, x^{3}+(1-a) x^{2}-\left(a^{2}+2\right) x+3 a-1=0$. Since in the case $1.2 .2, s_{2} \leq 1$, it forces $s_{1} \leq 1$, then $x=-1-a, y=-2 a$, so $(a-1)\left(a^{2}+4 a+1\right)=0$ follows from $x^{3}+(1-a) x^{2}-\left(a^{2}+2\right) x+3 a-1=0$, then $a=-2+\sqrt{3}, x=1-\sqrt{3}$, and we have $n=\sqrt{3}(n-s)$, but there are no such real numbers $n$ and $s$, it is a contradiction. So this combination is impossible. By symmetry, neither is the combination of Case 1.1.2-1.3.2.

Case 1.1.2-1.4.2:

$$
\left(\begin{array}{cc|cc}
x & 1 & a & 1 \\
1 & x & a & 1 \\
\hline a & a & y & \star \\
1 & 1 & \star & y
\end{array}\right)\left(\begin{array}{l}
\left(u_{1}, v_{1}\right) \\
\left(u_{2}, v_{2}\right)
\end{array}\right.
$$

By the results in Case 1.1.2 and 1.4.2, we have $x=2 a-1, y=a, a \in\left(0, \frac{1}{2}\right)$ or $x=-1-a, y=-2 a, a \in(-1,0)$ and then $y(x+1)=2$, it follows $a=1$ or $a=-1$, obviously this combination is impossible. 
Case 1.2.1-1.3.2:

$$
\left(\begin{array}{cc|cc}
x & 1 & a & 1 \\
1 & x & 1 & a \\
\hline a & 1 & x & \star \\
1 & a & \star & y
\end{array}\right)\left(\begin{array}{ll}
\left(u_{1}, v_{1}\right) \\
\left(u_{2}, v_{2}\right)
\end{array} .\right.
$$

By the result in Cases 1.2.1 and 1.3.2, we have $a \neq-1,(x-a)\left(x^{2}+a x-2\right)=0$ and $x^{3}+(1-a) x^{2}-\left(a^{2}+2\right) x+3 a-1=0$. If $x=a$, then $(a-1)^{2}(a+1)=0$, a contradiction; if $x^{2}=2-a x$, we have $0=x(2-a x)+(1-a)(2-a x)-\left(a^{2}+2\right) x+3 a-1=\left(a^{2}-a\right) x-a+1$, so $x=\frac{1}{a}$ then $x^{2}+a x-2=\frac{1}{a^{2}}-1=0$, a contradiction. So this combination is impossible. By symmetry, the combination of Case 1.2.2-1.3.1 is also impossible.

Case 1.2.1-1.4.2:

$$
\left(\begin{array}{cc|cc}
x & 1 & a & 1 \\
1 & x & 1 & 1 \\
\hline a & 1 & x & \star \\
1 & 1 & \star & y
\end{array}\right)\left(\begin{array}{ll}
\left(u_{1}, v_{1}\right) \\
\left(u_{2}, v_{2}\right)
\end{array}\right.
$$

By the results in Case 1.2.1 and 1.4.2, we have $a \neq-1,(x-a)\left(x^{2}+a x-2\right)=0$ and $x^{2}+(2-a) x-(a+1)=0$. If $x=a$ then $a^{2}+(2-a) a-(a+1)=0$, a contradiction; if $x^{2}+a x-2=0$ then $x=-\frac{1}{2}, a=-\frac{7}{2}$, a contradiction. So this combination is impossible. By symmetry, the combination of Case 1.3.1-1.4.2 is also impossible. 
By the discussion above Case 1.2.1-1.3.2 and Case 1.2.1-1.4.2 (which are impossible) and $x \neq 0$, so there is one possibility for Case 1.1.2-1.2.1-1.3.1 which has been illustrated as the first matrix in Theorem 3.2.2.

\section{Case 1.2.2-1.4.2:}

$$
\left(\begin{array}{ll|ll}
x & 1 & a & 1 \\
1 & x & 1 & 1 \\
\hline a & 1 & y & \star \\
1 & 1 & \star & y
\end{array}\right)\left(\begin{array}{ll}
\left(u_{1}, v_{1}\right) \\
\left(u_{2}, v_{2}\right)
\end{array} .\right.
$$

By the results in Case 1.2.2 and 1.4.2, we have $a \neq-1, x^{3}+(1-a) x^{2}-\left(a^{2}+2\right) x+$ $(3 a-1)=0$ and $x^{2}+(2-a) x-(a+1)=0$. It follows $(1+a) x=2$, obviously a contradiction and this combination is impossible. By symmetry, the combination of Case 1.3.2-1.4.2 is also impossible.

Case 1.2.2-1.3.2:

$$
\left(\begin{array}{ll|ll}
x & 1 & a & 1 \\
1 & x & 1 & a \\
\hline a & 1 & y & \star \\
1 & a & \star & y
\end{array}\right)\left(\begin{array}{ll}
\left(u_{1}, v_{1}\right) \\
\left(u_{2}, v_{2}\right)
\end{array} .\right.
$$

By the results in Case 1.2.2 and 1.3.2, we have $s_{2}=s_{3}=1$, and there is no other row 
allowed, this implies that $n=4$ and $x=\frac{a-1}{2}, a \neq-1$, then we have $5 a^{2}-12 a-1=0$ by $x^{3}+(1-a) x^{2}-\left(a^{2}+2\right) x+3 a-1=0$ then $a=\frac{6-\sqrt{41}}{5}$. Now

$$
\begin{aligned}
& \left(u_{1}+v_{1}\right)(1+x)=1+a \\
& \left(u_{1}-v_{1}\right)(1-x)=1-a .
\end{aligned}
$$

It follows that $B_{2}^{\prime}(3,4)=u_{1}+a v_{1}$ can neither be $a$ nor 1 , a contradiction. So this combination is impossible.

Thus we have discussed all the possible combinations for Case 1. The remainder of the proof above follows by applying a similar strategy for each of the 6 cases in $(3.30)$.

Recall that two matrices $A, B$, with the same order, are said to be $D$-similar if there is a diagonal matrix $D$ with diagonal entries equal to 1 or -1 such that $D A D=B$.

When $x=a=-1, y=1, n=2 s$, we use the notion of $D$-similarity and find the structure of a Mirsky matrix of the form

$$
\left(\begin{array}{cc}
-J_{s} & J_{s} \\
J_{s} & J_{s}
\end{array}\right) \text {. }
$$

We have performed some numerical experiments to find a Mirsky's matrix (see the algorithm in Appendix), and it seems that extensive random searching cannot find much more than the theory posted here. 


\subsection{Spread of Some Real Symmetric Matrices}

\subsubsection{The Form of Real Symmetric Matrices of Rank 2, with Entries $a$ or 1}

In this section, we study the structure of rank 2 symmetric matrices with entries $a$ or $1, a \in[-1,1)$.

Lemma 3.3.1 Let $A \in S_{n}-\{a, 1\}, a \in[-1,1)$, with $\operatorname{rank}(A)=2$. Without loss of generality, let the first and second row vector $\alpha, \beta$ be linearly independent. Then any remaining row $\gamma$ must lie in one of the following cases:

1. if $-1<a<1, a \neq-\frac{1}{2}$ and $a \neq 0, \gamma=\alpha$ or $\gamma=\beta$;

2. if $a=-1$, then $\gamma=\alpha, \gamma=\beta, \gamma=-\alpha$ or $\gamma=-\beta$;

3. if $a=-\frac{1}{2}$, then $\gamma=\alpha, \gamma=\beta$ or $\gamma=-\alpha-\beta$;

4. if $a=0$, then $\gamma=\alpha, \gamma=\beta$ or $\gamma=0$.

Proof. Since $\operatorname{rank}(A)=2$, the nonsingular upper left principal submatrix $B$ of order 2 will be one of cases below:

$$
\left(\begin{array}{ll}
a & 1 \\
1 & a
\end{array}\right)\left(\begin{array}{ll}
a & 1 \\
1 & 1
\end{array}\right) \quad\left(\begin{array}{ll}
1 & a \\
a & 1
\end{array}\right) \quad\left(\begin{array}{ll}
a & a \\
a & 1
\end{array}\right) .
$$

Case 1

Case 2

Case 3

Case 4

$$
a \neq-1 \quad a \neq-1 \quad a \neq 0
$$

Let $\gamma$ be an arbitrary remaining row vector in $A$ which we may assume is the $3^{\text {rd }}$ row by simultaneous row and column permutations, if necessary. Then $\gamma=u \alpha+v \beta, u, v \in \mathbb{R}$. 
We show the details for Case 2 in (3.33), similar arguments can be applied for the remaining cases.

\section{Case 2:}

$$
\left(\begin{array}{cc|c}
a & 1 & u a+v \\
1 & 1 & u+v \\
\hline u a+v & u+v & u^{2} a+2 u v+v^{2}
\end{array}\right) u
$$

The first principal submatrix of $A$, order 3 , is shown above, and $(u, v)$ is the pair of combination parameters for the $3^{\text {rd }}$ row.

$$
\begin{aligned}
& \text { If } \\
& \left\{\begin{array}{c}
u a+v=1 \\
u+v=1
\end{array} \Rightarrow(a-1) u=0, u=0, v=1 .\right.
\end{aligned}
$$

$$
\begin{aligned}
& \text { If } \\
& \left\{\begin{array}{c}
u a+v=a \\
u+v=a
\end{array} \Rightarrow(a-1) u=0, u=0, v=a, \text { thus } A(3,3)=a^{2},\right.
\end{aligned}
$$

If $a^{2}=1$, then $a=-1, u=0, v=-1$; if $a^{2}=a$, then $a=0, u=0, v=0$. 


$$
\begin{aligned}
& \text { If } \\
& \left\{\begin{array}{l}
u a+v=a \\
u+v=1
\end{array} \Rightarrow(u-1)(a-1)=0, u=1 \text { and } v=0\right. \text {. }
\end{aligned}
$$

$$
\begin{aligned}
& \text { If } \\
& \left\{\begin{array}{l}
u a+v=1 \\
u+v=a
\end{array} \Rightarrow(u+1)(a-1)=0, u=-1 \text { and } v=a+1, A(3,3)=u+a v\right. \text {. }
\end{aligned}
$$

If $u+a v=1, a^{2}+a-2=0$, then $a=1$ or -2 , a contradiction; if $u+a v=a$, then $a=-1, u=-1, v=0$.

We study all the possibilities for $3^{\text {rd }}$ row in $A$, and then the combinations of these possibilities in case 2. Applying this argument for the remaining cases, we can draw out the remaining conclusions in Lemma 3.3.1.

Note: By Lemma 3.3.1, when $-1<a<1, a \neq-\frac{1}{2}, a \neq 0$, and $\operatorname{rank}(A)=2$, then the remaining row $\gamma=\alpha$ or $\beta$, we let the numbers of rows equal to $\alpha$ and $\beta$ be $s$ and $(n-s)$, respectively $(1 \leq s \leq n-1)$. Then the structure of $A \in S_{n}-\{a, 1\}$ is shown as below (up to simultaneous row and column permutation): 


$$
\begin{array}{cc}
\left(\begin{array}{rc}
a J_{s} & J_{s \times(n-s)} \\
J_{(n-s) \times s} & a J_{n-s}
\end{array}\right), & \left(\begin{array}{rr}
a J_{s} & J_{s \times(n-s)} \\
& \\
J_{(n-s) \times s} & J_{n-s}
\end{array}\right), \\
\text { Type } 1 & \text { Type } 2 \\
\left(\begin{array}{cc}
J_{s} & a J_{s \times(n-s)} \\
a J_{(n-s) \times s} & J_{n-s}
\end{array}\right), & \left(\begin{array}{cc}
a J_{s} & a J_{s \times(n-s)} \\
a J_{(n-s) \times s} & J_{n-s}
\end{array}\right) . \\
\text { Type } 3 & \text { Type } 4
\end{array}
$$

Here Type 1 arises from Case 1 in Lemma 3.3.1, Type 2 from Case 2, Type 3 from Case 3 and Type 4 from Case 4.

When $a=-\frac{1}{2}, \operatorname{rank}(A)=2$, we let the first and second row vector $\alpha, \beta$ be linearly independent. Thus, any row $\gamma$ is equal to either $\alpha$ or $\beta$ for Case 2 in Lemma 3.3.1, and then we can get Type 2 in (3.34), if the number of rows $\alpha$ and $\beta$ is $s, n-s$ respectively $(1 \leq s \leq n-1)$.

For Case 1, Case 3 and Case 4 in Lemma 3.3.1, since $\gamma=\alpha, \gamma=\beta$ or $\gamma=-\alpha-\beta$. let the number of rows equal to $\alpha, \beta$ and $-\alpha-\beta$ be $s, t$ and $n-s-t$, respectively $(1 \leq s \leq n-1,1 \leq t \leq n-1, s+t \leq n)$, we can verify the structure of $A$, by simultaneous row and column permutations, illustrated below: 


$$
\left(\begin{array}{rrr}
-\frac{1}{2} J_{s} & J_{s \times t} & -\frac{1}{2} J_{s \times(n-s-t)} \\
J_{t \times s} & -\frac{1}{2} J_{t} & -\frac{1}{2} J_{t \times(n-s-t)} \\
-\frac{1}{2} J_{(n-s-t) \times s} & -\frac{1}{2} J_{(n-s-t) \times t} & J_{(n-s-t)}
\end{array}\right),
$$

Type 5

$$
\left(\begin{array}{rrr}
J_{s} & -\frac{1}{2} J_{s \times t} & -\frac{1}{2} J_{s \times(n-s-t)} \\
-\frac{1}{2} J_{t \times s} & J_{t} & -\frac{1}{2} J_{t \times(n-s-t)} \\
-\frac{1}{2} J_{(n-s-t) \times s} & -\frac{1}{2} J_{(n-s-t) \times t} & J_{(n-s-t)}
\end{array}\right) \text {, }
$$

Type 6

$$
\left(\begin{array}{rrr}
-\frac{1}{2} J_{s} & -\frac{1}{2} J_{s \times t} & J_{s \times(n-s-t)} \\
-\frac{1}{2} J_{t \times s} & J_{t} & -\frac{1}{2} J_{t \times(n-s-t)} \\
J_{(n-s-t) \times s} & -\frac{1}{2} J_{(n-s-t) \times t} & -\frac{1}{2} J_{(n-s-t)}
\end{array}\right) .
$$

Type 7

When $a=-1, \operatorname{rank}(A)=2$, without loss of generality, let the first and second row vectors $\alpha, \beta$ be linearly independent. Then any remaining row $\gamma$ is equal to either $\alpha, \beta,-\alpha$ or $-\beta$.

For Case $2, \gamma=\alpha, \beta,-\alpha$ or $-\beta$, let the number of rows equal to $\alpha, \beta,-\alpha$ and $-\beta$ be $s, t, s_{1}$ and $t_{1}$, respectively $\left(1 \leq s \leq n-1,1 \leq t \leq n-1, s+t+s_{1}+t_{1}=n\right)$. 
The structure of $A$ follows by simultaneous row and column permutations and is illustrated below:

$$
\left(\begin{array}{rrrr}
-J_{s} & J_{s \times s_{1}} & J_{s \times t} & -J_{s \times t_{1}} \\
J_{s_{1} \times s} & -J_{s_{1}} & -J_{s_{1} \times t} & J_{s_{1} \times t_{1}} \\
J_{t \times s} & -J_{t \times s_{1}} & J_{t} & -J_{t \times t_{1}} \\
-J_{t_{1} \times s} & J_{t_{1} \times s_{1}} & -J_{t_{1} \times t} & J_{t_{1}}
\end{array}\right),
$$

which is D-similar to

$$
\left(\begin{array}{rr}
-J_{s+s_{1}} & J_{\left(s+s_{1}\right) \times\left(t+t_{1}\right)} \\
J_{\left(t+t_{1}\right) \times\left(s+s_{1}\right)} & J_{t+t_{1}}
\end{array}\right) \text {. }
$$

Type 8

For Case 4 , we arrive at the same structure of $A$ as Type 8 , up to $D$-similarity and simultaneous row and column permutations.

When $a=0, \operatorname{rank}(A)=2$, without loss of generality, let the first and second row vector $\alpha, \beta$ be linearly independent. Then any remaining row $\gamma$ is equal to either $\alpha, \beta$ or 0 . Take the number of rows equal to $\alpha, \beta$ and 0 be $s, t$ and $n-s-t$, respectively $(1 \leq s \leq n-1,1 \leq t \leq n-1, s+t \leq n)$. The structure of $A$ then follows, by simultaneous row and column permutations, and is illustrated below: 


$$
\left(\begin{array}{rrr}
0_{s} & 0_{s \times(n-s-t)} & J_{s \times t} \\
0_{(n-s-t) \times s} & 0_{n-s-t} & 0_{(n-s-t) \times t} \\
J_{t \times s} & 0_{t \times(n-s-t)} & 0_{t}
\end{array}\right),
$$

Type 9

$$
\left(\begin{array}{rrr}
0_{s} & 0_{s \times(n-s-t)} & J_{s \times t} \\
0_{(n-s-t) \times s} & 0_{n-s-t} & 0_{(n-s-t) \times t} \\
J_{t \times s} & 0_{t \times(n-s-t)} & J_{t}
\end{array}\right),
$$

Type 10

$$
\left(\begin{array}{rrr}
0_{n-s-t} & 0_{(n-s-t) \times s} & 0_{(n-s-t) \times t} \\
0_{s \times(n-s-t)} & J_{s} & 0_{s \times t} \\
& 0_{t \times s} & J_{t}
\end{array}\right) .
$$

Type 11 


\subsubsection{The Spread of Real Symmetric Rank 2 Matrices with Entries $a$ or 1}

In the previous section, we studied the structure of real symmetric rank 2 matrices with entries $a$ or 1 , and now we focus on the maximum spread for these kinds of matrices.

Theorem 3.3.2 Let $A$ be a real symmetric $n \times n$ matrix, rank 2 , with entries a or 1 , that satisfy $-1<a<1, a \neq-\frac{1}{2}, a \neq 0, n \geq 2$. Then the following holds.

1. If the structure of $A$ is Type 1 in (3.34), then

$$
S P(A) \leq \begin{cases}n & \text { if } n \text { is even } \\ \sqrt{n^{2}+a^{2}-1} & \text { if } n \text { is odd. }\end{cases}
$$

If $n$ is even, equality holds if and only if $s=\frac{n}{2}$. If $n$ is odd, equality holds if and only if $s=\frac{n-1}{2}$ or $\frac{n+1}{2}$.

2. If the structure of $A$ is Type 2 in (3.34), $S P(A) \leq \sqrt{\frac{4}{a+3}}$. When $s=\operatorname{round}\left(\frac{n}{a+3}\right)$, the spread of $A$ is maximum. (Here round $(x)$ means an integer number closest to $x$.)

3. If the structure of $A$ is Type 3 in (3.34), $S P(A) \leq \frac{\sqrt{(n-2)^{2}+4 a^{2}(n-1)}+n}{2}$, equality holds if and only if $s=1$ or $s=n-1$. 
4. If the structure of $A$ is Type 4 in (3.34),

$$
S P(A) \leq\left\{\begin{array}{lr}
2 n \sqrt{a^{3} /(3 a+1)} & \text { if }-1<a<-\frac{1}{2}, \\
\sqrt{n^{2}+2\left(2 a^{2}-a-1\right) n-\left(3 a^{2}-2 a-1\right)} & \text { if }-\frac{1}{2}<a<-\frac{1}{3}, \\
\sqrt{n^{2}-8 n / 9} & \text { if } a=-\frac{1}{3}, \\
\sqrt{n^{2}+2\left(2 a^{2}-a-1\right) n-\left(3 a^{2}-2 a-1\right)} & \text { if }-\frac{1}{3}<a<0, \\
\left(a+n-1+\sqrt{n^{2}+2\left(2 a^{2}-a-1\right) n-\left(3 a^{2}-2 a-1\right)}\right) / 2 & \text { if } 0<a<1 .
\end{array}\right.
$$

Proof. For Type 1, we have

$$
A=\left(\begin{array}{rc}
a J_{s} & J_{s \times(n-s)} \\
J_{(n-s) \times s} & a J_{n-s}
\end{array}\right),
$$

and if we let $f(\lambda)=\operatorname{det}(\lambda I-A)=0$ be the characteristic polynomial of $A$. Then $f(\lambda)=0$ can be rewritten as $\lambda^{n-2}\left[\lambda^{2}-n a \lambda+s(n-s)\left(a^{2}-1\right)\right]=0$. Since $s(n-s)\left(a^{2}-\right.$ 1) $\leq 0$, we find

$$
S P(A)=\sqrt{(n a)^{2}-4 s(n-s)\left(a^{2}-1\right)}=\sqrt{-4\left(1-a^{2}\right) s^{2}+4 n\left(1-a^{2}\right) s+n^{2} a^{2}},
$$

and since $1-a^{2}>0$, so $S P(A) \leq n$. Furthermore, if $n$ is even, equality holds if and only if $s=\frac{n}{2}$; whereas if $n$ is odd, $S P(A) \leq \sqrt{n^{2}+a^{2}-1}$, equality holds if and only if $s=\frac{n-1}{2}$ or $s=\frac{n+1}{2}$. In any event, we have $S P(A) \leq n$ as $a^{2}<1$.

For Type 2, we have

$$
A=\left(\begin{array}{rc}
a J_{s} & J_{s \times(n-s)} \\
J_{(n-s) \times s} & J_{n-s}
\end{array}\right) .
$$


Then $f(\lambda)=0$ can be rewritten as $\lambda^{n-2}\left[\lambda^{2}-(a s+n-s) \lambda+s(n-s)(a-1)\right]=0$. Since $s(n-s)(a-1) \leq 0$, we find $S P(A)=\sqrt{(a s+n-s)^{2}+4 s(n-s)(1-a)}=\sqrt{(a+3)(a-1) s^{2}+2 n(1-a) s+n^{2}}$, and $(a+3)(a-1)<0$, so $S P(A) \leq \sqrt{\frac{4}{a+3}} n$. In this situation, we know $h(a)=\frac{4}{3+a}$ is a monotone decreasing function. So $1<\sqrt{h(a)}<\sqrt{2}$. This implies that the maximum spread for matrices of Type 2 is bigger than the maximum spread for matrices of Type 1, for the same value of $a$.

For Type 3, we have

$$
A=\left(\begin{array}{rc} 
& \\
J_{s} & a J_{s \times(n-s)} \\
& J_{n-s}
\end{array}\right) .
$$

Then $f(\lambda)=0$ can be rewritten as $\lambda^{n-2}\left[\lambda^{2}-n \lambda+s(n-s)\left(1-a^{2}\right)\right]=0$. By the conditions $s(n-s)\left(1-a^{2}\right)>0$ and $n>0$, we find

$$
S P(A)=\frac{\sqrt{n^{2}-4(n-s) s\left(1-a^{2}\right)}+n}{2}=\frac{\sqrt{4\left(1-a^{2}\right) s^{2}-4 n\left(1-a^{2}\right) s+n^{2}}+n}{2} .
$$

Obviously, $S P(A)$ is maximum when $s=1$ or $s=n-1$ and $S P(A) \leq \frac{\sqrt{(n-2)^{2}+4 a^{2}(n-1)}+n}{2}$ as $g\left(a^{2}\right)=\frac{\sqrt{(n-2)^{2}+4 a^{2}(n-1)}+n}{2}$ is a monotone increasing function and $g\left(a^{2}\right)<n$. In this event, we have that the maximum spread for matrices of Type 2 is bigger than the maximum spread for matrices of Type 3, for the same value of $a$. 
For Type 4, we have

$$
A=\left(\begin{array}{rc}
a J_{s} & a J_{s \times(n-s)} \\
a J_{(n-s) \times s} & J_{n-s}
\end{array}\right) .
$$

Then $f(\lambda)=0$ can be rewritten as $\lambda^{n-2}\left[\lambda^{2}-(s a+n-s) n \lambda+s(n-s) a(1-a)\right]=0$.

If $a<0$, then $s(n-s) a(1-a)<0$, and

$$
S P(A)=\sqrt{(s a+n-s)^{2}-4 s(n-s) a(1-a)}
$$

If $a>0$, then $s(n-s) a(1-a)>0$ and $s a+n-s>0$, then

$$
S P(A)=\frac{s a+n-s+\sqrt{(s a+n-s)^{2}-4 s(n-s) a(1-a)}}{2} .
$$

Furthermore, we find that:

When $-1<a<-\frac{1}{2}$, it implies that $\frac{2 a+1}{3 a+1} n \in\left(0, \frac{n}{2}\right)$. So $S P(A)$ is maximum if and only if $s=\operatorname{round}\left(\frac{2 a+1}{3 a+1} n\right)$ and $S P(A) \leq 2 n \sqrt{\frac{a^{3}}{3 a+1}}$. In this situation, we need to compare $h_{1}(a)=\frac{4 a^{3}}{3 a+1}$ to $h(a)=\frac{4}{a+3},-1<a<-\frac{1}{2}$, since $h(a)-h_{1}(a)=\frac{4\left(1-a^{2}\right)\left(1+a^{2}+3 a\right)}{(a+3)(3 a+1)}>0$, which implies that for the same $a \in\left(-1,-\frac{1}{2}\right)$, the maximum spread for matrices of Type 2 is bigger than the maximum spread for matrices of Type 4 .

When $-\frac{1}{2}<a<-\frac{1}{3}$, we have $\frac{2 a+1}{3 a+1} n<0$. Then $S P(A)$ is maximum if and only if $s=1$ and

$$
S P(A) \leq \sqrt{n^{2}+2\left(2 a^{2}-a-1\right) n-\left(3 a^{2}-2 a-1\right)} .
$$


When $a=-\frac{1}{3},-2 n(1-a)(1+2 a)<0$. So $S P(A)$ is maximum if and only if $s=1$ and

$$
S P(A) \leq \sqrt{n^{2}-\frac{8 n}{9}}<n
$$

When $-\frac{1}{3}<a<0$ we have $\frac{2 a+1}{3 a+1} n \in(n, \infty)$. So $S P(A)$ is maximum if and only if $s=1$ and

$$
S P(A) \leq \sqrt{n^{2}+2\left(2 a^{2}-a-1\right) n-\left(3 a^{2}-2 a-1\right)} .
$$

Define the function

$$
\begin{aligned}
h_{2}(a) & =\sqrt{n^{2}+2\left(2 a^{2}-a-1\right) n-\left(3 a^{2}-2 a-1\right)} \\
& =\sqrt{(4 n-3) a^{2}-(2 n-1) a+(n-1)^{2}}, \quad-\frac{1}{2}<a<0 .
\end{aligned}
$$

Then $h_{2}(a)$ is a monotone decreasing function. Hence $h_{2}(a)<h_{2}\left(-\frac{1}{2}\right)=\sqrt{n^{2}-\frac{3}{4}}<$ n. In this event, we have that for the same $a \in\left(-\frac{1}{2}, 0\right)$, the maximum spread for matrices of Type 2 is bigger than the maximum spread for matrices of Type 4.

When $0<a<1, \frac{2 a+1}{3 a+1} n \in\left(\frac{3}{4} n, n\right)$ and $s(a-1)<0$. So $S P(A)$ is maximum if and only if $s=1$ and

$$
S P(A) \leq \frac{a+n-1+\sqrt{n^{2}+2\left(2 a^{2}-a-1\right) n-\left(3 a^{2}-2 a-1\right)}}{2} .
$$

Define the function $h_{3}(a)=\frac{a+n-1+\sqrt{n^{2}+2\left(2 a^{2}-a-1\right) n-\left(3 a^{2}-2 a-1\right)}}{2}$, then $h_{3}(a)<n$ when $0<a<1$, which implies that for the same $a \in(0,1)$, the maximum spread for matrices of Type 2 is bigger than the maximum spread for matrices of Type 4. 
When the entry $a=-\frac{1}{2}$, we have the following theorem on the maximum spread of the matrices with rank 2 in $S_{n}-\{a, 1\}$.

Theorem 3.3.3 Let $A$ be a real symmetric $n \times n$ matrix of rank 2 , with entries 1 or $a=-\frac{1}{2}$. Then the following holds:

1. If the structure of $A$ is Type 5 in (3.35), $S P(A) \leq n$. Further, equality holds if and only if $s=t$.

2. If the structure of $A$ is Type 2 in (3.34), then $S P(A) \leq \sqrt{\frac{8}{5}} n$. When $s=\operatorname{round}\left(\frac{2 n}{5}\right)$, the spread of $A$ is maximum.

3. If the structure of $A$ is Type 6 in (3.35), $S P(A) \leq \frac{n+\sqrt{n^{2}-3(n-1)}}{2}$. Further, equality holds if and only if $s=1, t=n-1$ or $s=n-1, t=1$.

4. If the structure of $A$ is Type 7 in (3.35), $S P(A) \leq n$. Further, equality holds if and only if $n-t=2 s$.

Proof. For Type 5, we have

$$
A=\left(\begin{array}{rrr}
-\frac{1}{2} J_{s} & J_{s \times t} & -\frac{1}{2} J_{s \times(n-s-t)} \\
J_{t \times s} & -\frac{1}{2} J_{t} & -\frac{1}{2} J_{t \times(n-s-t)} \\
-\frac{1}{2} J_{(n-s-t) \times s} & -\frac{1}{2} J_{(n-s-t) \times t} & J_{n-s-t}
\end{array}\right) .
$$


Then $f(\lambda)=0$ can be rewritten as $\lambda^{n-2}\left[\lambda^{2}+\frac{3 s+3 t-2 n}{2} \lambda+\frac{3}{4}((s+t)(s+t-n)-s t)\right]=0$.

Since $(s+t)(s+t-n)-s t<0$, then

$$
S P(A)=\sqrt{\frac{(3 s+3 t-2 n)^{2}}{4}-3[(s+t)(s+t-n)-s t]}=\sqrt{\frac{-3(s-t)^{2}+4 n^{2}}{4}} .
$$

$S P(A)$ is maximum when $s=t, 1 \leq s \leq \frac{n}{2}$ and $S P(A) \leq n$.

For Type 2, by the related characteristic polynomial function, $f(\lambda)=0$, we have $S P(A) \leq \sqrt{\frac{8}{5}} n$. The maximum spread of $A$ attains when $s=\operatorname{round}\left(\frac{2 n}{5}\right)$ and it is bigger than the maximum spread for matrices of Type 5.

For Type 6

$$
A=\left(\begin{array}{rrr}
J_{s} & -\frac{1}{2} J_{s \times t} & -\frac{1}{2} J_{s \times(n-s-t)} \\
-\frac{1}{2} J_{t \times s} & J_{t} & -\frac{1}{2} J_{t \times(n-s-t)} \\
-\frac{1}{2} J_{(n-s-t) \times s} & -\frac{1}{2} J_{(n-s-t) \times t} & J_{n-s-t}
\end{array}\right) .
$$

Then $f(\lambda)=0$ can be rewritten as $\lambda^{n-2}\left[\lambda^{2}-n \lambda+\frac{3}{4}((s+t)(n-s-t)+s t)\right]=0$. Since $(s+t)(n-s-t)+s t>0$, then

$$
S P(A)=\frac{1}{2}\left(n+\sqrt{n^{2}-3 n(s+t)+3(s+t)^{2}-3 s t}\right) .
$$

Define $F_{1}(s, t)=n^{2}-3 n(s+t)+3(s+t)^{2}-3 s t$ be a function of $s, t$, which are two integer numbers in $[1, n-1], s+t \leq n$. Then we have

$$
\left\{\begin{array}{l}
\frac{\partial F_{1}}{\partial s}=-3 n+6(s+t)-3 s=0 \\
\frac{\partial F_{1}}{\partial t}=-3 n+6(s+t)-3 t=0
\end{array}\right.
$$


From (3.38) we have $s=t=\frac{n}{3}$. This is the only extreme minimum value of $F_{1}(s, t)$, and the maximum value is attained on the boundary of a region formed by lines $s \geq$ $1, t \geq 1, s+t \leq n$. Since $s, t$ are symmetric in $F_{1}(s, t)$, it's convenient to assume $s \leq t$, and $s+t=n$ on the boundary. This implies that if $F_{1}(s, t)=n^{2}-3 s t \leq n^{2}-3(n-1)$, then $S P(A) \leq \frac{1}{2}\left(n+\sqrt{n^{2}-3(n-1)}\right)$. Equality holds if and only if $s=1, t=n-1$ or $s=n-1, t=1$. It is easy to see that $\frac{1}{2}\left(n+\sqrt{n^{2}-3(n-1)}\right)<n$ and it also shows that the maximum spread for matrices of Type 2 is bigger than the maximum spread for matrices of Type 6 .

For Type 7

$$
A=\left(\begin{array}{rrr}
-\frac{1}{2} J_{s} & -\frac{1}{2} J_{s \times t} & J_{s \times(n-s-t)} \\
-\frac{1}{2} J_{t \times s} & J_{t} & -\frac{1}{2} J_{t \times(n-s-t)} \\
J_{(n-s-t) \times s} & -\frac{1}{2} J_{(n-s-t) \times t} & -\frac{1}{2} J_{n-s-t}
\end{array}\right) .
$$

Then $f(\lambda)=0$ can be rewritten as $\lambda^{n-2}\left[\lambda^{2}-\frac{1}{2}(3 t-n) \lambda+\frac{3}{4}\left(s^{2}-(n-t)(s+t)\right)\right]=0$. Since $s^{2}-(n-t)(s+t) \leq 0$, then

$$
S P(A)=\sqrt{\frac{1}{4}(3 t-n)^{2}-3\left[s^{2}-(n-t)(s+t)\right]}
$$

Define $F_{2}(s, t)=\frac{1}{4}(3 t-n)^{2}-3\left[s^{2}-(n-t)(s+t)\right]$ be a function of $s, t$, which are two integer numbers in $[1, n-1]$ with $s+t \leq n$. Then we have

$$
\left\{\begin{array}{l}
\frac{\partial F_{2}}{\partial s}=-6 s+3(n-t)=0 \\
\frac{\partial F_{2}}{\partial t}=\frac{3}{2}(3 t-n)+3(n-s-2 t)=0
\end{array}\right.
$$


From (3.39) we have $n-t=2 s$. It is the only maximum value of $f(s, t)$. So $S P(A) \leq n$, and equality holds if and only if $s=t$. Then it shows that the maximum spread of matrices for Type 2 is bigger than the maximum spread of matrices for Type 7 .

In the following theorem, it's convenient to denote $s, t$ as $s+s_{1}, t+t_{1}$.

Theorem 3.3.4 Let $A$ be a real symmetric $n \times n$ matrix of rank 2 , with entries 1 or $a=-1$. If the structure of $A$ is Type 8 in (3.36), then

$$
S P(A) \leq \begin{cases}\sqrt{2} n & \text { if } n \text { is even } \\ \sqrt{2 n^{2}-1} & \text { if } n \text { is odd. }\end{cases}
$$

Equality holds if and only if $s=t=\frac{n}{2}$, when $n$ is even; $s=\frac{n-1}{2}, t=\frac{n+1}{2}$ or $s=\frac{n+1}{2}, t=\frac{n-1}{2}$ when $n$ is odd.

Proof. Since

$$
A=\left(\begin{array}{cc}
-J_{s} & J_{s \times t} \\
J_{t \times s} & J_{t}
\end{array}\right)
$$

Then $f(\lambda)=0$ can be rewritten as $\lambda^{n-2}\left[\lambda^{2}+(s-t) \lambda-2 s t\right]=0$. Since $s t>0$, then

$$
S P(A)=\sqrt{(s-t)^{2}+8 s t}=\sqrt{-4 s^{2}+4 n s+n^{2}} .
$$

So $S P(A) \leq \sqrt{2} n$. Further, when $n$ is even, equality holds if and only if $s=t=\frac{n}{2}$; and when $n$ is odd, equality holds if and only if $s=\frac{n-1}{2}, t=\frac{n+1}{2}$ or $s=\frac{n+1}{2}, t=\frac{n-1}{2}$ and $S P(A)=\sqrt{2 n^{2}-1}$. In fact, by Theorem 2.2 .3 , we know the conclusion holds since $\|A\|_{F}=n$ in this situation. 
Theorem 3.3.5 Let $A$ be a real symmetric $n \times n$ matrix of rank 2 , with entries 1 or $a=0$. Then the following holds.

1. If the structure of $A$ is Type 9 in (3.37), then

$$
S P(A) \leq \begin{cases}n & \text { if } n \text { is even, } \\ \sqrt{n^{2}-1} & \text { if } n \text { is odd. }\end{cases}
$$

Equality holds if and only if $s=t=\frac{n}{2}$, when $n$ is even; $s=\frac{n-1}{2}, t=\frac{n+1}{2}$ or $s=\frac{n+1}{2}, t=\frac{n-1}{2}$, when $n$ is odd.

2. If the structure of $A$ is Type 10 in (3.37), then $S P(A) \leq \frac{2 n}{\sqrt{3}}$.

3. If the structure of $A$ is Type 11 in (3.37), then $S P(A) \leq n-1$.

Proof. For Type 9, we have

$$
A=\left(\begin{array}{rrr}
0_{s} & 0_{s \times(n-s-t)} & J_{s \times t} \\
0_{(n-s-t) \times s} & 0_{n-s-t} & 0_{(n-s-t) \times t} \\
J_{t \times s} & 0_{t \times(n-s-t)} & 0_{t}
\end{array}\right) .
$$

Then $f(\lambda)=0$ can be rewritten as $\lambda^{n-2}\left(\lambda^{2}-s t\right)=0$. Since $-s t<0$, then

$$
S P(A)=2 \sqrt{s t} \leq(s+t)^{2} \leq n^{2} .
$$

Obviously when $n$ is even, equality holds if and only if $s=t=\frac{n}{2}$; and when $n$ is odd, $S P(A)$ is maximum if and only if $s=\frac{n+1}{2}, t=\frac{n-1}{2}$ or $s=\frac{n-1}{2}, t=\frac{n+1}{2}$. The 
maximum value is $\sqrt{n^{2}-1}$. It shows that the maximum spread for matrices of Type 2 is bigger than the maximum spread for matrices of Type 9.

For Type 10, we have

$$
A=\left(\begin{array}{rrr}
0_{s} & 0_{s \times(n-s-t)} & J_{s \times t} \\
0_{(n-s-t) \times s} & 0_{n-s-t} & 0_{(n-s-t) \times t} \\
J_{t \times s} & 0_{t \times(n-s-t)} & J_{t}
\end{array}\right) .
$$

Then $f(\lambda)=0$ can be rewritten as $\lambda^{n-2}\left(\lambda^{2}-t \lambda-t s\right)=0$. Since $-s t<0$, then $S P(A)=\sqrt{t^{2}+4 s t}$. Let $s+t=m \leq n$, and define $f_{2}(s)=t^{2}+4 s t=-3 s^{2}+2 m s+m^{2}$, where $f_{2}(s)$ is a function of $s$. Obviously, $f(s) \leq \frac{4}{3} m^{2} \leq \frac{4}{3} n^{2}$, equality holds if and only if $m=n, s=\frac{n}{3}$. So when $s=\operatorname{round}\left(\frac{n}{3}\right), t=n-s, S P(A)$ is maximum. This shows that the maximum spread for matrices of Type 2 is bigger than the maximum spread for matrices of Type 10 .

For Type 11, suppose $1 \leq s \leq t \leq n-1$ for convenience, now we have

$$
A=\left(\begin{array}{rrr}
0_{n-s-t} & 0_{(n-s-t) \times s} & 0_{(n-s-t) \times t} \\
0_{s \times(n-s-t)} & J_{s} & 0_{s \times t} \\
0_{t \times(n-s-t)} & 0_{t \times s} & J_{t}
\end{array}\right) .
$$

Then $f(\lambda)=0$ can be rewritten as $\lambda^{n-2}(\lambda-t)(\lambda-s)=0$. Since $-s t<0$, then $S P(A)=t \leq n-1$. Equality holds if and only if $s=1, t=n-1$. It shows that the maximum spread for matrices of Type 2 is bigger than the maximum spread for 
matrices of Type 11 .

From the theorems in this section, we know that the maximum spread is attained by some matrices of Type 2 .

From some numerical experiments, we verify the above four theorems and find for any rank 2 matrix $A \in S_{n}-\{a, 1\}, a \in[-1,1)$, when its structure is same as that in Type 2, then its spread is maximum. The followings are the examples for $n=4$ and 5.

Examples:

- $n=4$ :

$$
\left(\begin{array}{rrrr}
-0.9 & -0.9 & 1.0 & 1.0 \\
-0.9 & -0.9 & 1.0 & 1.0 \\
1.0 & 1.0 & 1.0 & 1.0 \\
1.0 & 1.0 & 1.0 & 1.0
\end{array}\right), \quad\left(\begin{array}{rrrr}
-0.5 & -0.5 & 1.0 & 1.0 \\
-0.5 & -0.5 & 1.0 & 1.0 \\
1.0 & 1.0 & 1.0 & 1.0 \\
1.0 & 1.0 & 1.0 & 1.0
\end{array}\right),
$$

if $a=-0.9$, then $s=2, S P(A)=5.5172 ;$ if $a=-0.5$, then $s=2, S P(A)=$ 5.0000 .

$$
\left(\begin{array}{llll}
0.1 & 1.0 & 1.0 & 1.0 \\
1.0 & 1.0 & 1.0 & 1.0 \\
1.0 & 1.0 & 1.0 & 1.0 \\
1.0 & 1.0 & 1.0 & 1.0
\end{array}\right), \quad\left(\begin{array}{llll}
0.0 & 1.0 & 1.0 & 1.0 \\
1.0 & 1.0 & 1.0 & 1.0 \\
1.0 & 1.0 & 1.0 & 1.0 \\
1.0 & 1.0 & 1.0 & 1.0
\end{array}\right),
$$

if $a=0.1$, then $s=1, S P(A)=4.5177$; if $a=0.0$, then $s=1, S P(A)=$ 4.5826 . 
- $n=5$ :

$$
\left(\begin{array}{lllll}
0.0 & 0.0 & 1.0 & 1.0 & 1.0 \\
0.0 & 0.0 & 1.0 & 1.0 & 1.0 \\
1.0 & 1.0 & 1.0 & 1.0 & 1.0 \\
1.0 & 1.0 & 1.0 & 1.0 & 1.0 \\
1.0 & 1.0 & 1.0 & 1.0 & 1.0
\end{array}\right),
$$

if $a=0.0$, then $s=2, S P(A)=5.7446$.

$$
\left(\begin{array}{rrrrr}
-1.0 & -1.0 & 1.0 & 1.0 & 1.0 \\
-1.0 & -1.0 & 1.0 & 1.0 & 1.0 \\
1.0 & 1.0 & 1.0 & 1.0 & 1.0 \\
1.0 & 1.0 & 1.0 & 1.0 & 1.0 \\
1.0 & 1.0 & 1.0 & 1.0 & 1.0
\end{array}\right)
$$

if $a=-1.0$, then $s=2, S P(A)=7.0000$.

$$
\left(\begin{array}{rrrrr}
-0.5 & -0.5 & 1.0 & 1.0 & 1.0 \\
-0.5 & -0.5 & 1.0 & 1.0 & 1.0 \\
1.0 & 1.0 & 1.0 & 1.0 & 1.0 \\
1.0 & 1.0 & 1.0 & 1.0 & 1.0 \\
1.0 & 1.0 & 1.0 & 1.0 & 1.0
\end{array}\right)
$$

if $a=-0.5$, then $s=2, S P(A)=6.3246$. 


\subsection{Conclusion about Real Symmetric Matrices}

\subsubsection{Maximum Spread of Real Symmetric Rank 2 Matrices with Entries in the Interval $[a, 1] \quad(-1 \leq a<1)$}

From the work in Section 3.3, we arrive at our main contribution involving the maximum spread for rank 2 matrices in $S_{n}[a, 1]$.

Theorem 3.4.1 Let $A \in S_{n}[a, 1], a \in[-1,1)$ and $\operatorname{rank}(A)=2$, if $S P(A)$ is maximum, then $A$ must be in the following form (up to permutation similarity):

$$
A=\left(\begin{array}{cc}
a J_{s_{0}} & J_{s_{0} \times\left(n-s_{0}\right)} \\
J_{\left(n-s_{0}\right) \times s_{0}} & J_{n-s_{0}}
\end{array}\right)
$$

and

$$
\left\{\begin{array}{l}
s_{0}=\operatorname{round}\left(\frac{n}{a+3}\right) \\
S P(A)=\sqrt{\left(a^{2}+2 a-3\right) s_{0}^{2}+2 n(1-a) s_{0}+n^{2}}
\end{array}\right.
$$

Theorem 3.4.2 If the symmetric real matrix is

$$
A=\left(\begin{array}{cc}
a J_{s_{0}} & J_{s_{0} \times\left(n-s_{0}\right)} \\
J_{\left(n-s_{0}\right) \times s_{0}} & J_{n-s_{0}}
\end{array}\right),
$$

with unit vectors $X=\left(x_{1}, \ldots, x_{n}\right)^{T}, Y=\left(y_{1}, \ldots, y_{n}\right)^{T}$ being the eigenvectors corresponding to the eigenvalues $\lambda_{1}, \lambda_{n}$ of $A$, respectively, then

$$
x_{i} x_{j}-y_{i} y_{j} \neq 0, i, j \in\{1,2 \ldots, n\}
$$


Proof. Since $\operatorname{det}(\lambda I-A)=0$, we know $\lambda_{1}, \lambda_{n}$ must satisfy

$$
\lambda^{2}-\left(n+(a-1) s_{0}\right) \lambda+(a-1)\left(n-s_{0}\right) s_{0}=0
$$

so

$$
\left\{\begin{aligned}
\lambda_{1}+\lambda_{n} & =n+(a-1) s_{0} \\
\lambda_{1} \lambda_{n} & =(a-1)\left(n-s_{0}\right) s_{0},
\end{aligned}\right.
$$

and $A X=\lambda_{1} X, A Y=\lambda_{n} Y$. We can rewrite these equations as $A Z=\lambda_{i} Z, Z=X$, if $i=1 ; Z=Y$, if $i=n$, where $Z=\left(z_{1}, \ldots, z_{s_{0}}, z_{s_{0}+1}, \ldots, z_{n}\right)^{T}, \frac{n}{4}<s_{0} \leq \frac{n}{2}, n \geq 2$. Thus

$$
a \sum_{1}^{s_{0}} z_{t}+\sum_{s_{0}+1}^{n} z_{t}=\lambda_{i} z_{m}, m=1, \ldots, s_{0}
$$

and

$$
\sum_{1}^{s_{0}} z_{t}+\sum_{s_{0}+1}^{n} z_{t}=\lambda_{i} z_{r}, r=s_{0}+1, \ldots, n .
$$

Since $\lambda_{1}>0>\lambda_{n}$ it follows that

$$
\begin{aligned}
& z_{1}=\cdots=z_{s_{0}}, z_{s_{0}+1}=\cdots=z_{n}, \\
& \lambda_{i} z_{n}=\left(\lambda_{i}+(1-a) s_{0}\right) z_{1}, \\
& \lambda_{1}+(1-a) s_{0}>0 .
\end{aligned}
$$

If $\lambda_{n}+(1-a) s_{0}=0$, then $y_{n}=0$, which implies $y_{1}=0$ by $s_{0} y_{1}+\left(n-s_{0}\right) y_{n}=\lambda_{n} y_{n}$. Thus $Y=0$, a contradiction. Since $\|Z\|_{2}=1$, we have

$$
z_{1}^{2}=\frac{\lambda_{i}^{2}}{s_{0} \lambda_{i}^{2}+\left(n-s_{0}\right)\left(\lambda_{i}+(1-a) s_{0}\right)^{2}}, \quad z_{n}=\frac{\lambda_{i}+(1-a) s_{0}}{\lambda_{i}} z_{1}
$$


and

$$
\begin{aligned}
X^{T} Y & =s_{0} x_{1} y_{1}+\left(n-s_{0}\right) \frac{\lambda_{1}+(1-a) s_{0}}{\lambda_{1}} x_{1} \frac{\lambda_{n}+(1-a) s_{0}}{\lambda_{n}} y_{1} \\
& =x_{1} y_{1}\left(s_{0}+\frac{\left(\lambda_{1}+(1-a) s_{0}\right)\left(\lambda_{n}+(1-a) s_{0}\right)}{\lambda_{1} \lambda_{n}}\left(n-s_{0}\right)\right)=0
\end{aligned}
$$

Without loss of generality, we suppose $x_{1}, y_{1}>0$, then we prove $x_{i} x_{j}-y_{i} y_{j} \neq 0$ in different cases:

(1) $i, j \in\left\{1, \ldots, s_{0}\right\}$

(2) $i, j \in\left\{s_{0}+1, \ldots, n\right\}$;

(3) $i \in\left\{1, \ldots, s_{0}\right\}, j \in\left\{s_{0}+1, \ldots, n\right\}$ or $j \in\left\{1, \ldots, s_{0}\right\}, i \in\left\{s_{0}+1, \ldots, n\right\}$.

In case (1):

$$
\begin{aligned}
x_{i} x_{j}-y_{i} y_{j} & =x_{1}^{2}-y_{1}^{2} \\
& =\frac{\lambda_{1}^{2}}{s_{0} \lambda_{1}^{2}+\left(n-s_{0}\right)\left(\lambda_{1}+(1-a) s_{0}\right)^{2}}-\frac{\lambda_{n}^{2}}{s_{0} \lambda_{n}^{2}+\left(n-s_{0}\right)\left(\lambda_{n}+(1-a) s_{0}\right)^{2}},
\end{aligned}
$$

because

$$
\begin{aligned}
& \lambda_{1}^{2}\left(s_{0} \lambda_{n}^{2}+\left(n-s_{0}\right)\left(\lambda_{n}+(1-a) s_{0}\right)^{2}\right)-\lambda_{n}^{2}\left(s_{0} \lambda_{1}^{2}+\left(n-s_{0}\right)\left(\lambda_{1}+(1-a) s_{0}\right)^{2}\right) \\
= & \left(n-s_{0}\right)\left[\left(\lambda_{1} \lambda_{n}+\lambda_{1}(1-a) s_{0}\right)^{2}-\left(\lambda_{1} \lambda_{n}+\lambda_{1}(1-a) s_{0}\right)^{2}\right] \\
= & \left(n-s_{0}\right)(1-a) s_{0}\left(\lambda_{1}-\lambda_{n}\right)\left(2 \lambda_{1} \lambda_{n}+(1-a) s_{0}\left(\lambda_{1}+\lambda_{n}\right)\right) \\
= & -\left(n-s_{0}\right)^{2}(1-a)^{2} s_{0}^{2}\left(\lambda_{1}-\lambda_{n}\right)<0,
\end{aligned}
$$

we have $x_{i} x_{j}-y_{i} y_{j} \neq 0$. 
In case (2):

$$
\begin{aligned}
x_{i} x_{j}-y_{i} y_{j} & =x_{n}^{2}-y_{n}^{2} \\
& =\frac{\left(\lambda_{1}+(1-a) s_{0}\right)^{2}}{\lambda_{1}^{2}} x_{1}^{2}-\frac{\left(\lambda_{n}+(1-a) s_{0}\right)^{2}}{\lambda_{n}^{2}} y_{1}^{2} \\
& =\frac{\left(\lambda_{1}+(1-a) s_{0}\right)^{2}}{s_{0} \lambda_{1}^{2}+\left(n-s_{0}\right)\left(\lambda_{1}+(1-a) s_{0}\right)^{2}}-\frac{\left(\lambda_{n}+(1-a) s_{0}\right)^{2}}{s_{0} \lambda_{n}^{2}+\left(n-s_{0}\right)\left(\lambda_{n}+(1-a) s_{0}\right)^{2}}
\end{aligned}
$$

because

$$
\begin{aligned}
& \left(\lambda_{1}+(1-a) s_{0}\right)^{2}\left(s_{0} \lambda_{n}^{2}+\left(n-s_{0}\right)\left(\lambda_{n}+(1-a) s_{0}\right)^{2}\right) \\
& \quad-\left(\lambda_{n}+(1-a) s_{0}\right)^{2}\left(s_{0} \lambda_{1}^{2}+\left(n-s_{0}\right)\left(\lambda_{1}+(1-a) s_{0}\right)^{2}\right) \\
& =s_{0}\left[\left(\lambda_{1} \lambda_{n}+\lambda_{n}(1-a) s_{0}\right)^{2}-\left(\lambda_{1} \lambda_{n}+\lambda_{1}(1-a) s_{0}\right)^{2}\right] \\
= & s_{0}(1-a) s_{0}\left(\lambda_{n}-\lambda_{1}\right)\left(2 \lambda_{1} \lambda_{n}+(1-a) s_{0}\left(\lambda_{1}+\lambda_{n}\right)\right) \\
= & s_{0}^{3}(1-a)^{2}\left(\lambda_{n}-\lambda_{1}\right)\left(s_{0}(1+a)-n\right),
\end{aligned}
$$

since $\frac{n}{a+3} \leq \frac{n}{2}, 1+a<2$, then $s_{0}(1+a)<n$ and $x_{i} x_{j}-y_{i} y_{j} \neq 0$.

In case (3): by symmetry, without loss of generality, suppose $i \in\left\{1, \ldots, s_{0}\right\}, j \in$ $\left\{s_{0}+1, \ldots, n\right\}$, then

$$
\begin{aligned}
x_{i} x_{j}-y_{i} y_{j} & =x_{1} x_{n}-y_{1} y_{n} \\
& =\frac{\lambda_{1}+(1-a) s_{0}}{\lambda_{1}} x_{1}^{2}-\frac{\lambda_{n}+(1-a) s_{0}}{\lambda_{n}} y_{1}^{2},
\end{aligned}
$$


because

$$
\begin{aligned}
& \left(\lambda_{1}^{2}+(1-a) s_{0} \lambda_{1}\right)\left(s_{0} \lambda_{n}^{2}+\left(n-s_{0}\right)\left(\lambda_{n}+(1-a) s_{0}\right)^{2}\right) \\
& \quad-\left(\lambda_{n}^{2}+(1-a) s_{0} \lambda_{n}\right)\left(s_{0} \lambda_{1}^{2}+\left(n-s_{0}\right)\left(\lambda_{1}+(1-a) s_{0}\right)^{2}\right) \\
& =(1-a) s_{0}\left(\lambda_{n}-\lambda_{1}\right)\left[s_{0} \lambda_{1} \lambda_{n}-\left(n-s_{0}\right)\left(2 \lambda_{1} \lambda_{n}+(1-a) s_{0}\left(\lambda_{1}+\lambda_{n}\right)\right)\right. \\
& \left.\quad+\left(n-s_{0}\right)\left(\lambda_{1} \lambda_{n}-(1-a)^{2} s_{0}^{2}\right)\right] \\
& =2\left(n-s_{0}\right)(1-a)^{2} s_{0}^{3}\left(\lambda_{1}-\lambda_{n}\right)
\end{aligned}
$$

we have $x_{i} x_{j}-y_{i} y_{j} \neq 0$. 


\subsubsection{Maximum Spread of Real Symmetric Matrices with Entries in the Interval $[a, b]$}

In the previous section, we studied the case, a real symmetric matrix $A$ with entries in an interval and $\operatorname{rank}(A)=2$. We know that if $\operatorname{rank}(A)=1$, then the related spread can not be maximum. We have performed many numerical experiments (see the Appendix) to find the maximum spread for a real symmetric matrix, and the results of these experiments suggest the following conjecture.

Conjecture 3.4.3 For the class of real symmetric matrices $A$ with entries in the interval $[a, b]$, the maximum spread in the class must be attained by a real symmetric matrix $S_{n}-\{a, b\}$ of $\operatorname{rank}(A)=2$.

Case 1: If $a^{2} \leq b^{2}$, let $u=a b^{-1}$. Then we have that the maximum value $S P(A)$ of spread

$$
b \sqrt{\left(u^{2}+2 u-3\right) s^{2}+2 n(1-u) s+n^{2}}
$$

where $s=\operatorname{round}\left(\frac{n}{u+3}\right)$, and by row and column permutation the structure of $A$ is given by

$$
\left(\begin{array}{rr}
a J_{s} & b J_{s \times(n-s)} \\
b J_{(n-s) \times s} & b J_{n-s}
\end{array}\right) \text {. }
$$

Case 2: If $a^{2}>b^{2}$, let $u=b a^{-1}$, then we have that the maximum value $S P(A)$ of 
spread

$$
-a \sqrt{\left(u^{2}+2 u-3\right) s^{2}+2 n(1-u) s+n^{2}}
$$

where $s=\operatorname{round}\left(\frac{n}{u+3}\right)$, and by row and column permutation the structure of $A$ is given by

$$
\left(\begin{array}{rc}
b J_{s} & a J_{s \times(n-s)} \\
a J_{(n-s) \times s} & a J_{n-s}
\end{array}\right) .
$$

We have tried to compare the spreads between the real symmetric matrices with entries in an interval of rank more than or equal to 2. And even for the rank 3 case, we can not determine sufficient information about the distribution of the eigenvalues to derive any definitive conclusions. For example, let

$$
A=\left(\begin{array}{ccc}
a & 1 & 1 \\
1 & 1 & 1 \\
1 & 1 & 1
\end{array}\right) \text { and its perturbation } A(\epsilon)=\left(\begin{array}{ccc}
a+\epsilon & 1 & 1 \\
1 & 1-\epsilon & 1 \\
1 & 1 & 1-\epsilon
\end{array}\right),
$$

where $a \in[-1,1)$ and $\epsilon>0$. So now $\operatorname{rank}(A(\epsilon))=3$, but it is complicated to compare its spread with $S P(A)$ since the characteristic polynomial of $A(\epsilon)$ is a cubic function of $\epsilon$ and it is difficult to describe the associated spread of $A(\epsilon)$. We know that $S P(A(\epsilon) \leq S P(A)+2 \epsilon$ by Weyl's Perturbation Theorem [30], but we can not prove that $S P(A(\epsilon))<S P(A)$. 


\subsection{Real Skew Symmetric Matrices}

In this section, we let $S S_{n}[-a, a]$ be the set of real skew symmetric matrices with entries in the interval $[-a, a]$. It is well known that for a real skew symmetric matrix, all of the nonzero eigenvalues are purely imaginary, and thus are of the form $i \lambda_{1},-i \lambda_{1}, i \lambda_{2},-i \lambda_{2}, \ldots$, where each $\lambda_{k}$ is real.

Theorem 3.5.1 Suppose $A \in S S_{n}[-a, a]$ and $\operatorname{rank}(A)=2$. If spread of $A, S P(A)$, is maximum, then each off-diagonal entry of $A$ is either $a$ or $-a$.

Proof. If $\operatorname{rank}(A)=2$, and suppose there are two conjugate complex eigenvalues with corresponding eigenvectors $q_{1}, q_{2}$. Let

$$
U=Q\left(\begin{array}{cc:c}
\frac{1}{\sqrt{2}} & \frac{1}{\sqrt{2}} & O_{2 \times(n-2)} \\
\frac{i}{\sqrt{2}} & \frac{-i}{\sqrt{2}} & \\
\hdashline O_{(n-2) \times 2} & I_{(n-2) \times(n-2)}
\end{array}\right) \in \mathscr{U}_{n} .
$$

Then $u_{1}=\frac{1}{\sqrt{2}} q_{1}+\frac{i}{\sqrt{2}} q_{2}, u_{2}=\frac{1}{\sqrt{2}} q_{1}-\frac{i}{\sqrt{2}} q_{2}$, and

$$
U^{*} A U=\left(\begin{array}{cc:c}
i \lambda_{1} & 0 & O_{2 \times(n-2)} \\
0 & -i \lambda_{1} & \ldots \ldots \ldots \\
\hdashline O_{(n-2) \times 2} & O_{(n-2) \times(n-2)}
\end{array}\right) .
$$

Then

$$
S P(A)=2\left|\lambda_{1}\right|=\left|u_{1}^{*} A u_{1}-u_{2}^{*} A u_{2}\right|=\left|q_{1}^{T} A q_{2}-q_{2}^{T} A q_{1}\right|=\left|e^{T}\left[A \circ\left(q_{1} q_{2}^{T}-q_{2} q_{1}^{T}\right)\right] e\right|
$$

Note that $B=q_{1} q_{2}^{T}-q_{2} q_{1}^{T}$ is a skew symmetric matrix. From this we define a new rank 2 normal matrix $\tilde{A}=\left(\tilde{a}_{i j}\right)$ as $\tilde{a}_{i j}=a$ if $q_{1 i} q_{2 j}-q_{2 i} q_{1 j}>0$, otherwise $\tilde{a}_{i j}=-a$ if $q_{1 i} q_{2 j}-q_{2 i} q_{1 j}<0$. It follows that $S P(A) \leq S P(\tilde{A})$. 
By considering $a^{-1} A$ instead of $A$, we need only study the maximum spread in $S S_{n}[-1,1]$

\section{Notes:}

1. Let $\mathbb{F} \in\{\mathbb{R}, \mathbb{C}\}$, and $X \in \mathbb{F}$ be a convex and compact set. Define

$$
\begin{array}{r}
\mathscr{H}_{n}(X)=\left\{a=\left[\alpha_{i j}\right] \in M_{n}(\mathbb{F}) \mid a^{*}=a \& \alpha_{i j} \in X, \forall i, j\right\}, \\
\mathscr{S} \mathscr{H}_{n}(X)=\left\{b=\left[\beta_{i j}\right] \in M_{n}(\mathbb{F}) \mid b^{*}=-b \& \beta_{i j} \in X, \forall i, j\right\} .
\end{array}
$$

Define the exterior, ext $X$, as the set of all exterior points of $X$, then we have the following proposition.

Proposition 3.5.2 $\mathscr{H}_{n}(X) \& \mathscr{S}_{H_{n}}(X)$ are both compact and convex sets.

Moreover,

$$
\begin{gathered}
a \in \operatorname{ext} \mathscr{H}_{n}(X) \Leftrightarrow \alpha_{i j} \in \operatorname{ext} X, \forall i, j \\
\& b \in \operatorname{ext} \mathscr{S} \mathscr{H}_{n}(X) \Leftrightarrow \beta_{i j} \in \operatorname{ext} X, \forall i, j .
\end{gathered}
$$

The proof is straightforward.

2. For $a \in \mathscr{H}_{n}(X)$, let $\lambda^{\downarrow}(a)=n$-tuple of eigenvalues of $a$, in descending order. So, $\lambda^{\downarrow}(a) \in \mathbb{R}^{n}$

Recall Weyl's Perturbation Theorem: $\forall$ Hermitian $a, b \in M_{n}(\mathbb{F})$,

$$
\max _{1 \leq j \leq n}\left|\lambda_{j}^{\downarrow}(a)-\lambda_{j}^{\downarrow}(b)\right| \leq\|a-b\|, \text { where }\|\cdot\| \text { is an operator norm. }
$$

Hence, the map $\lambda_{j}^{\downarrow}: \mathscr{H}_{n}(X) \rightarrow \mathbb{R}$ is continuous, $\forall 1 \leq j \leq n$. In particular, the spread $S P=\lambda_{1}^{\downarrow}-\lambda_{n}^{\downarrow}=\lambda_{\max }-\lambda_{\min }$ is continuous. 
As, $\mathscr{H}_{n}(X)$ is convex, it is path connected, and thus,

$$
\left\{S P(a) \mid a \in \mathscr{H}_{n}(X)\right\} \text { is a closed interval in } \mathbb{R} \text {. }
$$

Because $\mathscr{S} \mathscr{H}_{n}(X)=i \mathscr{H}_{n}(-i X)$, the same ideas apply, but we do need to take into account that the diagonal entries are all equal to zero. That is,

Proposition 3.5.3 $\left\{S P(a) \mid a \in \mathscr{H}_{n}(X)\right\}$ and $\left\{S P(b) \mid b \in \mathscr{S} \mathscr{H}_{n}(X)\right\}$ are closed intervals.

3. A function $f: C \rightarrow \mathbb{R}$, where $C$ is a convex set in a vector space, is

(i) convex, if $f(t x+(1-t) y) \leq t f(x)+(1-t) f(y), \forall x, y \in C, t \in[0,1]$

(ii) concave, if $-f$ is convex.

Note that $\lambda_{\text {max }}: \mathscr{H}_{n}(X) \rightarrow \mathbb{R}$ is convex, and $\lambda_{\min }: \mathscr{H}_{n}(X) \rightarrow \mathbb{R}$ is concave. Hence, the spread $S P: \mathscr{H}_{n}(X) \rightarrow \mathbb{R}$ is a convex function. By Bauer's Maximum principle, the continuous convex function $S P$ attains its maximum at an extreme point of $\mathscr{H}_{n}(X)$. Hence, if $a \in \mathscr{H}_{n}(X)$ satisfies $S P(a) \geq S P\left(a^{\prime}\right), \forall a^{\prime} \in$ $\mathscr{H}_{n}(X)$ then $\alpha_{i j} \in \operatorname{ext} X, \forall i, j$. The above discussion offers another viewpoint and proof of Lemma 3.2.1 and Theorem 3.5.1, and the above analysis was the result of some communication from Dr. Douglas Farenick.

Now we focus on rank 2 matrices $A \in S S_{n}[-1,1]$. As before there must exist an invertible principal submatrix of order 2 in any real skew symmetric matrix of rank 2. Assume that this submatrix lies in the upper left block by simultaneous permutation 
of rows and columns of $A$. Therefore any other row must be a linear combination of the first two rows. As a result we can determine the structure of these matrices.

Examples for such matrices with a pair of conjugate complex eigenvalues are :

$$
A_{2}=\left(\begin{array}{cc}
0 & -1 \\
1 & 0
\end{array}\right), \quad A_{3}=\left(\begin{array}{ccc}
0 & -1 & -1 \\
1 & 0 & -1 \\
1 & 1 & 0
\end{array}\right), \quad A_{4}=\left(\begin{array}{rrrr}
0 & 0 & -1 & -1 \\
0 & 0 & -1 & -1 \\
1 & 1 & 0 & -1 \\
1 & 1 & 1 & 0
\end{array}\right)
$$




\subsubsection{Structure of Real Skew Symmetric Matrices Whose Spreads Attain the Bound in the Mirsky's Theorem}

It is evident that the structure of an invertible principal submatrix of a real skew symmetric matrix $A=\left[a_{i j}\right]$ with off diagonal entries $-1,1$ or 0 is the matrix $A_{2}$ above. Furthermore the spread of this matrix attains the bound in Theorem 2.2.3 when its rank is 2 .

Now, if necessary, assume $A_{2}$ to the upper left corner of $A$ by row and column permutations and therefore the other row vectors of $A$ must be linear combinations of the first and second rows of $A$. Let $r_{1}, r_{2}$ represent rows 1,2 of $A$. For $i=3, \ldots, n$, each row $i$ must be of the form $\alpha_{i} r_{1}+\beta_{i} r_{2}$ where $\left(\alpha_{i}, \beta_{i}\right)$ is the related real combination parameters. We know $\left|\alpha_{i}\right| \leq 1,\left|\beta_{i}\right| \leq 1$ and they are distributed in a circle with radius $r_{i}$ and center 0 , where $0 \leq r_{i} \leq \sqrt{2}, 3 \leq i \leq n$. Further $\alpha_{i}=r_{i} \cos \theta_{i}, \beta_{i}=r_{i} \sin \theta_{i}$, where $\theta_{i}$ is the related argument. Now $a_{i j}=\alpha_{j} \beta_{i}-\alpha_{i} \beta_{j}=r_{i} r_{j} \sin \left(\theta_{i}-\theta_{j}\right), 3 \leq i, j \leq$ n. Without loss of generality, assume $r_{i} \leq r_{j}, 3 \leq i \leq j \leq n$, then we have the following results.

Claim 3.5.4 If $0 \leq r_{i}, r_{j} \leq 1$, then if and only if $r_{i}=r_{j}=1$ and $\left|\theta_{i}-\theta_{j}\right|=$ $k \pi \pm \frac{\pi}{2}, k \in \mathbb{Z}$ then $\left|a_{i j}\right|=1$ and $\|A\|_{F}^{2}$ attains the maximum value.

Claim 3.5.5 If $0 \leq r_{i} \leq 1 \leq r_{j} \leq \sqrt{2}$, then if and only if $r_{i}=1, r_{j}=\sqrt{2}$ and $\left|\theta_{i}-\theta_{j}\right|=k \pi \pm \frac{\pi}{4}, k \in \mathbb{Z}$ then $\left|a_{i j}\right|=1$ and $\|A\|_{F}^{2}$ attains the maximum value.

Claim 3.5.6 If $1<r_{i} \leq r_{j} \leq \sqrt{2},\left|a_{i 1}\right|^{2}+\left|a_{i 2}\right|^{2}+\left|a_{j 1}\right|^{2}+\left|a_{j 2}\right|^{2}+\left|a_{i j}\right|^{2} \leq 4$ if and only if $r_{i}=r_{j}=\sqrt{2}$ and $\theta_{i}, \theta_{j}$ are in collinear, equality holds. 


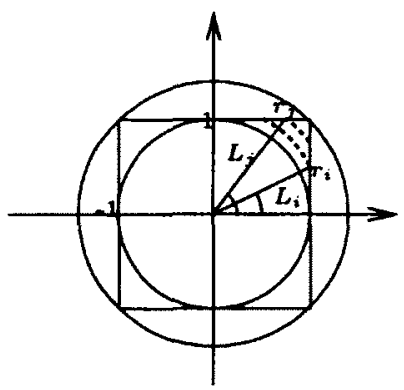

Case 1

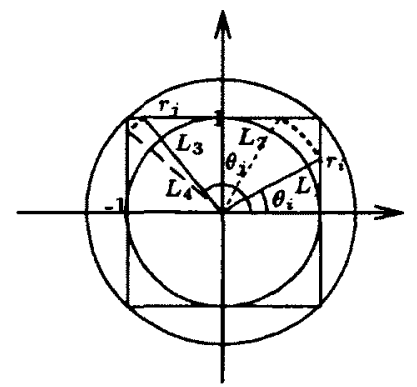

Case 2

Figure 3.1: Two cases of the combination parameters in real skew symmetric matrices

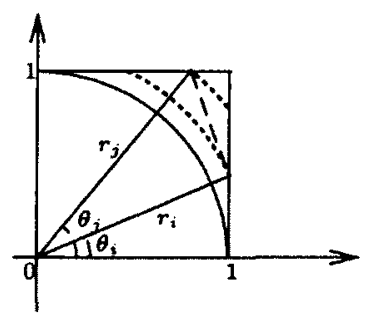

Figure 3.2: Case for both arguments in quadrant I

Proof. Without loss of generality, assume $\theta_{i}$ in quadrant I, if $\theta_{j}$ is in III or IV, then $\left|a_{i j}\right|$ keeps the same value as argument $\theta_{j}$ is in I or II. There are then just two cases to consider.

Case 1: $\left(\alpha_{i}, \beta_{i}\right),\left(\alpha_{j}, \beta_{j}\right)$ are both in quadrant I. Then by the Figure 3.2 above, we have

$$
\left|a_{i j}\right|=r_{i} r_{j} \sin \left(\theta_{j}-\theta_{i}\right) \leq 1-\sqrt{\left(r_{i}^{2}-1\right)\left(r_{j}^{2}-1\right)}<1
$$




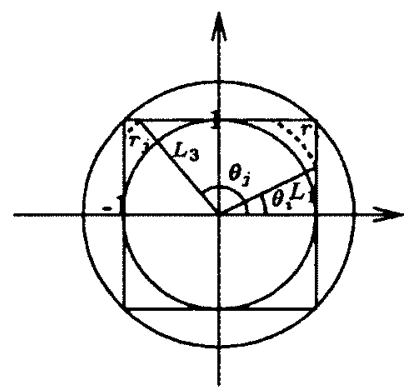

Subcase 1

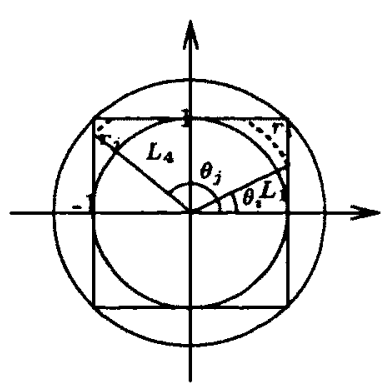

Subcase 2

Figure 3.3: Subcases 1 and 2

Thus

$$
\begin{aligned}
& \left|a_{i 1}\right|^{2}+\left|a_{i 2}\right|^{2}+\left|a_{j 1}\right|^{2}+\left|a_{j 2}\right|^{2}+\left|a_{i j}\right|^{2} \\
= & r_{i}^{2}+r_{j}^{2}+1+\left(r_{i}^{2}-1\right)\left(r_{j}^{2}-1\right)-2 \sqrt{\left(r_{i}^{2}-1\right)\left(r_{j}^{2}-1\right)} \\
\leq & 2+r_{i}^{2} r_{j}^{2}-2\left(r_{i}^{2}-1\right) \\
= & 4+r_{i}^{2}\left(r_{j}^{2}-2\right) \\
\leq & 4
\end{aligned}
$$

and equality holds if and only if $r_{i}=r_{j}=\sqrt{2}$.

Case 2: $\left(\alpha_{i}, \beta_{i}\right)$ is in quadrant I, and $\left(\alpha_{j}, \beta_{j}\right)$ is in II, and there are four subcases total to consider.

Subcase 1: See Figure 3.3 above. If $\theta_{i}$ is located in $L_{1}$ and $\theta_{j}$ in $L_{3}$, then

$$
\left|a_{i j}\right|=r_{i} r_{j} \sin \left(\theta_{j}-\theta_{i}\right)=1+\sqrt{\left(r_{i}^{2}-1\right)\left(r_{j}^{2}-1\right)}>1
$$

which contradicts the constraints that the absolute value of entries are at most 1 . 
Subcase 2: See Figure 3.3 above. If $\theta_{i}$ is located in $L_{1}$ and $\theta_{j}$ in $L_{4}$, then

$$
\left|a_{i j}\right|=\sqrt{\left(r_{i}^{2}-1\right)}+\sqrt{\left(r_{j}^{2}-1\right)} \leq 1
$$

which implies that $r_{j} \leq \frac{\sqrt{5}}{2}$. Then

$$
\begin{aligned}
& \left|a_{i 1}\right|^{2}+\left|a_{i 2}\right|^{2}+\left|a_{j 1}\right|^{2}+\left|a_{j 2}\right|^{2}+\left|a_{i j}\right|^{2} \\
= & r_{i}^{2}+r_{j}^{2}+\left(\sqrt{r_{i}^{2}-1}+\sqrt{r_{j}^{2}-1}\right)^{2} \\
\leq & 2 \times \frac{5}{4}+1 \\
& <4
\end{aligned}
$$

Note: By subcase 1 and subcase 2 , we have $\left(\theta_{j}-\theta_{i}\right) \in\left[\frac{\pi}{2}, \pi\right)$, and $\left|a_{i j}\right|$ decreases as $\left(\theta_{j}-\theta_{i}\right)$ increases. When $\theta_{j}$ is located between $L_{3}$ and $L_{4}, \theta_{i}$ in $L_{1}$. If $a_{i j}=1$, then this forces $r_{i} \leq r_{j}<\frac{\sqrt{5}}{2}$, so $\left|a_{i 1}\right|^{2}+\left|a_{i 2}\right|^{2}+\left|a_{j 1}\right|^{2}+$ $\left|a_{j 2}\right|^{2}+\left|a_{i j}\right|^{2}=r_{i}^{2}+r_{j}^{2}+1<4$.

Subcase 3: If $\theta_{i}$ is located in $L_{2}$ and $\theta_{j}$ in $L_{3}$, then

$$
\left|a_{i j}\right|=\sqrt{\left(r_{i}^{2}-1\right)}+\sqrt{\left(r_{j}^{2}-1\right)} \leq 1
$$

which implies that $r_{j} \leq \frac{\sqrt{5}}{2}$, as in subcase 2 .

Subcase 4: If $\theta_{i}$ is located in $L_{2}$ and $\theta_{j}$ in $L_{4}$, then

$$
\left|a_{i j}\right|=1+\sqrt{\left(r_{i}^{2}-1\right)\left(r_{j}^{2}-1\right)}>1
$$

which is a contradiction.

Note: By subcase 3 and subcase 4 , we have $\left(\theta_{j}-\theta_{i}\right) \in(0, \pi)$, and $\left|a_{i j}\right|$ increases to 1 , then is larger than 1 , and then decreases back to 1 , as $\left(\theta_{j}-\theta_{i}\right)$ 
increases. When $\theta_{j}$ is located between $L_{3}$ and $L_{4}, \theta_{i}$ in $L_{2}$, if $a_{i j}=1$ then this forces that $r_{j}<\frac{\sqrt{5}}{2}$, so $\left|a_{i 1}\right|^{2}+\left|a_{i 2}\right|^{2}+\left|a_{j 1}\right|^{2}+\left|a_{j 2}\right|^{2}+\left|a_{i j}\right|^{2}=r_{i}^{2}+r_{j}^{2}+1<$ 4.

Equality holds if and only if $r_{i}=r_{j}=\sqrt{2}$ and $\theta_{i}, \theta_{j}$ are collinear. In this case we have $\left|a_{i 1}\right|^{2}+\left|a_{i 2}\right|^{2}+\left|a_{j 1}\right|^{2}+\left|a_{j 2}\right|^{2}+\left|a_{i j}\right|^{2}=4$ and $a_{i j}=0$, without loss of generality we let $\theta_{i}=\theta_{j}=\frac{\pi}{4}$.

Theorem 3.5.7 If $A=\left[a_{i j}\right] \in S S_{n}[-1,1]$, and $\operatorname{rank}(A)=2$, with $\left|a_{i j}\right| \leq 1,1 \leq$ $i, j \leq n$, then $S P(A)$ is maximum if and only if $A$ is permutationally similar or D-similar to

$$
\left(\begin{array}{ccc}
0_{r \times r} & -J_{r \times s} & -J_{r \times t} \\
J_{s \times r} & 0_{s \times s} & -J_{s \times t} \\
J_{t \times r} & J_{t \times s} & 0_{t \times t}
\end{array}\right)
$$

where $1 \leq r, s<n$ and $r+s+t=n, r, s, t \in \mathbb{N}$. Moreover, in this case

$$
\max (S P(A))= \begin{cases}\frac{\sqrt{3}}{3}(2 n), & \text { if } n \equiv 0(\bmod 3), \\ \frac{\sqrt{3}}{3}\left(2 \sqrt{n^{2}-1}\right), & \text { if } n \equiv 1(\bmod 3), \text { or } n \equiv 2(\bmod 3) .\end{cases}
$$

To prove the above theorem, we need the following lemmas.

Lemma 3.5.8 Let $A=\left[a_{i j}\right] \in S S_{n}[-1,1], a_{i j}=0,1$ or $-1, \operatorname{rank}(A)=2$, then

$$
\max \left(\|A\|_{F}^{2}\right)= \begin{cases}6 k^{2} & \text { if } n=3 k, \\ 6 k^{2}+4 k & \text { if } n=3 k+1, \\ 6 k^{2}+8 k+2 & \text { if } n=3 k+2 .\end{cases}
$$


Proof. There is a principal submatrix $D$-similar or permutationally similar to $A_{2}$ when $\operatorname{rank}(A)=2$ set in the upper left corner of $A$. If $\|A\|_{F}^{2}$ attains the maximum value, and the remaining row vectors of $A$ are linear combinations of the first two row vectors of $A$, by the claims $3.5 .4,3.5 .5$, and 3.5 .6 , the combination parameters are $(1,0),(0,1)$ or $(1,1)$, and the related numbers of these parameters are $s_{1}, s_{2}$, and $s_{3}$, respectively, then $A$ is $D$-similar or permutationally similar to

$$
A=\left(\begin{array}{ccccc}
0 & -1 & 0_{1 \times s_{1}} & -J_{1 \times s_{2}} & -J_{1 \times s_{3}} \\
1 & 0 & J_{1 \times s_{1}} & 0_{1 \times s_{2}} & J_{1 \times s_{3}} \\
0_{s_{1} \times 1} & -J_{s_{1} \times 1} & 0_{s_{1} \times s_{1}} & -J_{s_{1} \times s_{2}} & -J_{s_{1} \times s_{3}} \\
J_{s_{2} \times 1} & 0_{s_{2} \times 1} & J_{s_{2} \times s_{1}} & 0_{s_{2} \times s_{2}} & J_{s_{2} \times s_{3}} \\
J_{s_{3} \times 1} & -J_{s_{3} \times 1} & J_{s_{3} \times s_{1}} & -J_{s_{3} \times s_{2}} & 0_{s_{3} \times s_{3}}
\end{array}\right)
$$

where $s_{1}, s_{2}, s_{3} \in[0, n-2], s_{1}+s_{2}+s_{3}=n-2, n \geq 3$.

So now

$$
\begin{aligned}
\|A\|_{F}^{2} & =2\left[1+s_{1}+s_{2}+2 s_{3}+s_{1} \times s_{2}+s_{1} \times s_{3}+s_{2} \times s_{3}\right] \\
& =2\left[-\left(s_{1}+s_{2}\right)^{2}+(n-3)\left(s_{1}+s_{2}\right)+(2 n-3)+s_{1} \times s_{2}\right] \\
& \leq-\frac{3}{2}\left(s_{1}+s_{2}\right)^{2}+2(n-3)\left(s_{1}+s_{2}\right)+(4 n-6) \\
& \leq 4 n-6+\frac{2}{3}(n-3)^{2}
\end{aligned}
$$


It follows that

$$
\max \left(\|A\|_{F}^{2}\right)= \begin{cases}6 k^{2}=\frac{2}{3} n^{2} & \text { if } n=3 k \\ & \text { and } s_{1}=s_{2}=k-1, s_{3}=k \\ 6 k^{2}+4 k=\frac{2}{3}\left(n^{2}-1\right) & \text { if } n=3 k+1, \\ & \text { and } s_{1}=s_{3}=k, s_{2}=k-1 \\ & \text { or } s_{1}=k-1, s_{2}=s_{3}=k \\ & \text { or } s_{1}=s_{2}=k-1, s_{3}=k+1 \\ & \text { if } n=3 k+2, \\ & \text { and } s_{1}=k, s_{2}=k-1, s_{3}=k+1 \\ & \text { or } s_{1}=k-1, s_{2}=k, s_{3}=k+1 \\ & \text { or } s_{1}=s_{2}=s_{3}=k .\end{cases}
$$

By necessary $D$-similarities or permutation similarities, from (3.43) $A$ is of the form

$$
A=\left(\begin{array}{ccc}
0_{r \times r} & -J_{r \times s} & -J_{r \times t} \\
J_{s \times r} & 0_{s \times s} & -J_{s \times t} \\
J_{t \times r} & J_{t \times s} & 0_{t \times t}
\end{array}\right),
$$

where $1 \leq r, s<n, r+s+t=n$.

Claim 3.5.9 If $|\alpha| \leq 1,|\beta| \leq 1,|\alpha-\beta| \leq 1, \alpha, \beta \in \mathbb{R}$, then $\alpha^{2}+\beta^{2}+(\alpha-\beta)^{2} \leq 2$. By the claims above equality holds if and only if the combination parameters $(\alpha, \beta)=$ $(1,0)$ or $(0,1)$ or $(1,1)$. 
Lemma 3.5.10 Let $A_{n}=\left[a_{i j}\right] \in S S_{n}[-1,1], \operatorname{rank}\left(A_{n}\right)=2$. Then $S P(A)$ is maximum if and only if there is a $2 \times 2$ invertible principal submatrix of $A$ which is D-similar to $A_{2}$. After necessary row and column permutations, assume $A_{2}$ is in the upper left corner of $A$. Then any other row vector of $A$ is a linear combination of the first two row vectors with combination parameters $\left(\alpha_{i}, \beta_{i}\right), i=3, \ldots, n$. By the claims above, these parameters are of the form $(1,0),(0,1),(1,1)$ and let the number of these parameters are $s_{1}, s_{2}, s_{3}$, respectively. Then

$$
\begin{array}{ll}
s_{1}=s_{2}=k-1, s_{3}=k, & \text { if } n=3 k ; \\
s_{1}=s_{3}=k, s_{2}=k-1, \text { or } & \\
s_{1}=k-1, s_{2}=s_{3}=k, \text { or } & \\
s_{1}=s_{2}=k-1, s_{3}=k+1, & \text { if } n=3 k+1 ; \\
s_{1}=k, s_{2}=k-1, s_{3}=k+1, \text { or } & \\
s_{1}=k-1, s_{2}=k, s_{3}=k+1, \text { or } & \\
s_{1}=s_{2}=s_{3}=k, & \text { if } n=3 k+2 .
\end{array}
$$

Proof. We use induction on $n$ to verify Lemma 3.5.8. By the claims above, when $n=2, A$ is $D$-similar to $A_{2}$ which has the maximum spread; and when $n=3, A$ is $D$-similar or permutationally similar to $A_{3}$ which has the maximum spread; and when $n=4, A$ is $D$-similar or permutationally similar to $A_{4}$ which has the maximum spread.

Now if $A_{3 k}$ has the structure of matrix (3.43), when $n$ increases to $3 k+1$, we assume the combination parameter for the last row vector is $\left(\alpha_{3 k+1}, \beta_{3 k+1}\right)$ and also that 
$s_{1}=s_{2}=k-1, s_{3}=k, k \geq 1$, and $s_{1}, s_{2}, s_{3} \in[0,3 k-2], s_{1}+s_{2}+s_{3}=3 k-2, n \geq 3$.

Then it follows that $A_{3 k+1}$ is of the form

$\left(\begin{array}{cc|c|c|c|c}0 & -1 & 0_{1 \times s_{1}} & -J_{1 \times s_{2}} & -J_{1 \times s_{3}} & -\beta_{3 k+1} \\ 1 & 0 & J_{1 \times s_{1}} & 0_{1 \times s_{2}} & J_{1 \times s_{3}} & \alpha_{3 k+1} \\ \hline 0_{s_{1} \times 1} & -J_{s_{1} \times 1} & 0_{s_{1} \times s_{1}} & -J_{s_{1} \times s_{2}} & -J_{s_{1} \times s_{3}} & -\beta_{3 k+1} J_{s_{1} \times 1} \\ \hline J_{s_{2} \times 1} & 0_{s_{2} \times 1} & J_{s_{2} \times s_{1}} & 0_{s_{2} \times s_{2}} & J_{s_{2} \times s_{3}} & \alpha_{3 k+1} J_{s_{2} \times 1} \\ \hline J_{s_{3} \times 1} & -J_{s_{3} \times 1} & J_{s_{3} \times s_{1}} & -J_{s_{3} \times s_{2}} & 0_{s_{3} \times s_{3}} & \left(\alpha_{3 k+1}-\beta_{3 k+1}\right) J_{s_{3} \times 1} \\ \hline \beta_{3 k+1} & -\alpha_{3 k+1} & \beta_{3 k+1} J_{1 \times s_{1}} & -\alpha_{3 k+1} J_{1 \times s_{2}} & \left(\beta_{3 k+1}-\alpha_{3 k+1}\right) J_{1 \times s_{3}} & 0\end{array}\right)$.

Now

$$
\begin{aligned}
\left\|A_{3 k+1}\right\|_{F}^{2} & =\left\|A_{3 k}\right\|_{F}^{2}+2\left[\beta_{3 k+1}^{2}\left(s_{1}+1\right)+\alpha_{3 k+1}^{2}\left(s_{2}+1\right)+\left(\alpha_{3 k+1}-\beta_{3 k+1}\right)^{2} s_{3}\right] \\
& =6 k^{2}+2 k\left[\alpha_{3 k+1}^{2}+\beta_{3 k+1}^{2}+\left(\alpha_{3 k+1}-\beta_{3 k+1}\right)^{2}\right] \\
& \leq 6 k^{2}+4 k .
\end{aligned}
$$

This implies that when $n=3 k+1$,

$$
\begin{aligned}
& s_{1}^{\prime}=k, s_{2}^{\prime}=k-1, s_{3}^{\prime}=k, \text { or } \\
& s_{1}^{\prime}=k-1, s_{2}^{\prime}=k, s_{3}^{\prime}=k, \text { or } \\
& s^{\prime} 1=k-1, s_{2}^{\prime}=k-1, s_{3}^{\prime}=k+1,
\end{aligned}
$$

where $s_{1}^{\prime}, s_{2}^{\prime}, s_{3}^{\prime}$, are numbers of combination parameters of $(1,0),(0,1)$ and $(1,1)$ in $A_{3 k+1}$.

If $A_{3 k+1}$ has the structure of matrix (3.43), when $n$ increases to $3 k+2$, we assume the combination parameter for the last row vector is $\left(\alpha_{3 k+2}, \beta_{3 k+2}\right)$. 
In this case if $s_{1}=k, s_{2}=k-1, s_{3}=k, k \geq 1$, then we have

$$
\begin{aligned}
\left\|A_{3 k+2}\right\|_{F}^{2} & =\left\|A_{3 k+1}\right\|_{F}^{2}+2\left[\beta_{3 k+2}^{2}\left(s_{1}+1\right)+\alpha_{3 k+2}^{2}\left(s_{2}+1\right)+\left(\alpha_{3 k+2}-\beta_{3 k+2}\right)^{2} s_{3}\right] \\
& =6 k^{2}+4 k+2\left[k \alpha_{3 k+2}^{2}+(k+1) \beta_{3 k+2}^{2}+k\left(\alpha_{3 k+2}-\beta_{3 k+2}\right)^{2}\right] \\
& =\left(6 k^{2}+4 k\right)+2 k\left[\alpha_{3 k+2}^{2}+\beta_{3 k+2}^{2}+\left(\alpha_{3 k+2}-\beta_{3 k+2}\right)^{2}\right]+2 \beta_{3 k+2}^{2} \\
& \leq 6 k^{2}+8 k+2
\end{aligned}
$$

Equality holds if and only if $\alpha_{3 k+2}=\beta_{3 k+2}=1$ or $\alpha_{3 k+2}=0, \beta_{3 k+2}=1$. This implies that when $n=3 k+2, s_{1}^{\prime}=k, s_{2}^{\prime}=k-1, s_{3}^{\prime}=k+1$, or $s_{1}^{\prime}=s_{2}^{\prime}=s_{3}^{\prime}=k, S P\left(A_{3 k+2}\right)$ attains the maximum possible value.

$$
\begin{aligned}
& \text { If } s_{1}=k-1, s_{2}=k, s_{3}=k, k \geq 1, \text { we have } \\
& \qquad \begin{aligned}
\left\|A_{3 k+2}\right\|_{F}^{2} & =\left\|A_{3 k+1}\right\|_{F}^{2}+2\left[\beta_{3 k+2}^{2}\left(s_{1}+1\right)+\alpha_{3 k+2}^{2}\left(s_{2}+1\right)+\left(\alpha_{3 k+2}-\beta_{3 k+2}\right)^{2} s_{3}\right] \\
& =6 k^{2}+4 k+2\left[(k+1) \alpha_{3 k+2}^{2}+k \beta_{3 k+2}^{2}+k\left(\alpha_{3 k+2}-\beta_{3 k+2}\right)^{2}\right] \\
& \leq 6 k^{2}+8 k+2 .
\end{aligned}
\end{aligned}
$$

Equality holds if and only if $\alpha_{3 k+2}=\beta_{3 k+2}=1$ or $\alpha_{3 k+2}=1, \beta_{3 k+2}=0$. This implies that when $n=3 k+2, s_{1}^{\prime}=k-1, s_{2}^{\prime}=k, s_{3}^{\prime}=k+1$, or $s_{1}^{\prime}=s_{2}^{\prime}=s_{3}^{\prime}=k, S P\left(A_{3 k+2}\right)$ attains the maximum possible value. 


$$
\begin{aligned}
& \text { If } s_{1}=s_{2}=k-1, s_{3}=k+1, k \geq 1, \text { we have } \\
& \qquad \begin{aligned}
\left\|A_{3 k+2}\right\|_{F}^{2} & =\left\|A_{3 k+1}\right\|_{F}^{2}+2\left[\beta_{3 k+2}^{2}\left(s_{1}+1\right)+\alpha_{3 k+2}^{2}\left(s_{2}+1\right)+\left(\alpha_{3 k+2}-\beta_{3 k+2}\right)^{2} s_{3}\right] \\
& =6 k^{2}+4 k+2\left[k \alpha_{3 k+2}^{2}+k \beta_{3 k+2}^{2}+(k+1)\left(\alpha_{3 k+2}-\beta_{3 k+2}\right)^{2}\right] \\
& \leq 6 k^{2}+8 k+2 .
\end{aligned}
\end{aligned}
$$

Equality holds if and only if $\alpha_{3 k+2}=1, \beta_{3 k+2}=0$ or $\alpha_{3 k+2}=0, \beta_{3 k+2}=1$. This implies that when $n=3 k+2, s_{1}^{\prime}=k-1, s_{2}^{\prime}=k, s_{3}^{\prime}=k+1$, or $s_{1}^{\prime}=s_{2}^{\prime}=s_{3}^{\prime}=$ $k, S P\left(A_{3 k+2}\right)$ attains the maximum possible value.

If $A_{3 k+2}$ has the structure of matrix (3.43), when $n$ increases to $3 k+3$, we assume the combination parameters for the last row vector is $\left(\alpha_{3 k+3}, \beta_{3 k+3}\right)$.

In this case if $s_{1}=k, s_{2}=k-1, s_{3}=k+1, k \geq 1$, then we have

$$
\begin{aligned}
\left\|A_{3 k+3}\right\|_{F}^{2} & =\left\|A_{3 k+2}\right\|_{F}^{2}+2\left[\beta_{3 k+3}^{2}\left(s_{1}+1\right)+\alpha_{3 k+3}^{2}\left(s_{2}+1\right)+\left(\alpha_{3 k+3}-\beta_{3 k+3}\right)^{2} s_{3}\right] \\
& =6 k^{2}+8 k+2+2\left[k \alpha_{3 k+3}^{2}+(k+1) \beta_{3 k+3}^{2}+(k+1)\left(\alpha_{3 k+3}-\beta_{3 k+3}\right)^{2}\right] \\
& \leq\left(6 k^{2}+8 k+2\right)+4(k+1) .
\end{aligned}
$$

Equality holds if and only if $\alpha_{3 k+3}=0, \beta_{3 k+3}=1$. This implies that when $n=$ $3 k+3, s_{1}^{\prime}=s_{2}^{\prime}=k, s_{3}^{\prime}=k+1, S P\left(A_{3 k+3}\right)$ attains the maximum possible value. 


$$
\begin{aligned}
& \text { If } s_{1}=s_{2}=s_{3}=k, k \geq 1 \text {, we have } \\
& \begin{aligned}
\left\|A_{3 k+3}\right\|_{F}^{2} & =\left\|A_{3 k+2}\right\|_{F}^{2}+2\left[\beta_{3 k+3}^{2}\left(s_{1}+1\right)+\alpha_{3 k+3}^{2}\left(s_{2}+1\right)+\left(\alpha_{3 k+3}-\beta_{3 k+3}\right)^{2} s_{3}\right] \\
& =\left(6 k^{2}+8 k+2\right)+2\left[(k+1) \alpha_{3 k+3}^{2}+(k+1) \beta_{3 k+3}^{2}+k\left(\alpha_{3 k+3}-\beta_{3 k+3}\right)^{2}\right] \\
& \leq\left(6 k^{2}+8 k+2\right)+4(k+1) .
\end{aligned}
\end{aligned}
$$

Equality holds if and only if $\alpha_{3 k+3}=\beta_{3 k+3}=1$. This implies that when $n=$ $3 k+3, s_{1}^{\prime}=s_{2}^{\prime}=k, s_{3}^{\prime}=k+1, S P\left(A_{3 k+3}\right)$ attains the maximum possible value.

By the induction on the order of $A$, if $S P(A)$ attains the maximum spread, then $A$ has the same structure as the matrix (3.43). It is evident that $A$ is $D$-similar or permutationally similar to

$$
\left(\begin{array}{ccc}
0_{r \times r} & -J_{r \times s} & -J_{r \times t} \\
J_{s \times r} & 0_{s \times s} & -J_{s \times t} \\
J_{t \times r} & J_{t \times s} & 0_{t \times t}
\end{array}\right)
$$

where $1 \leq r, s<n, r+s+t=n$, and

$$
\|A\|_{F}^{2}= \begin{cases}6 k^{2}=\frac{2}{3} n^{2} & \text { if } n=3 k \\ 6 k^{2}+4 k=\frac{2}{3}\left(n^{2}-1\right) & \text { if } n=3 k+1 \\ 6 k^{2}+8 k+2=\frac{2}{3}\left(n^{2}-1\right) & \text { if } n=3 k+2 .\end{cases}
$$

So $S P(A) \leq \frac{2 n}{\sqrt{3}}$. 


\subsection{Case of Complex Normal Matrices}

In this section, we focus on two classes of complex normal matrices with entries of modulus at most 1 . We use $\mathscr{H}_{n}$ to denote the class of $n \times n$ Hermitian matrices and $\mathscr{S} \mathscr{H}_{n}$ denote the class of $n \times n$ skew Hermitian matrices.

\subsubsection{Structure of Complex Hermitian Matrices Whose Spreads Attain the Bound in Mirsky's Theorem}

Let $A=\left[a_{i j}\right] \in \mathscr{H}_{n}(\mathbb{C}),\left\|a_{i j}\right\| \leq 1$, if $S P(A)$ attains the upper bound in Theorem 2.2.3 and $n$ is even, $\operatorname{rank}(A)=2$ and $\operatorname{tr}(A)=0$, then there is a $2 \times 2$ invertible principal submatrix after necessary row and column permutations,

$$
\left(\begin{array}{cc}
1 & a \\
\bar{a} & -1
\end{array}\right),
$$

in the upper left corner of $A$. Thus $A$ may be produced by the strategy that the remaining row vectors are combinations of the first 2 row vectors in $A$. Furthermore, $1,-1$ must occur along the main diagonal of $A$ in pairs. We can then derive the structure of $A$ by studying its principal submatrix. Suppose the $4 \times 4$ principal submatrix $A[\{1,2,2 m+1,2 m+2\} ;\{1,2,2 m+1,2 m+2\}], 1 \leq m \leq \frac{n}{2}-1$, has the form

$$
\left(\begin{array}{cc|cc}
1 & a & b & d \\
\bar{a} & -1 & c & e \\
\hline \bar{b} & \bar{c} & 1 & f \\
\bar{d} & \bar{e} & \bar{f} & -1
\end{array}\right)
$$


Let the $1^{\text {st }}, 2^{\text {nd }},(2 m+1)^{\text {th }},(2 m+2)^{\text {th }}$ row vectors be $\alpha, \beta, \gamma, \delta$ and $\gamma, \delta$ are combination of $\alpha, \beta$ with combination parameters $(s, t),(p, q)$, respectively. It follows that

$$
\left\{\begin{array}{c}
s+t \bar{a}=\bar{b} \\
s a-t=\bar{c} \\
s b+t c=1 \\
s d+t e=f
\end{array}\right.
$$

By (3.44), we have $t(c \bar{b}-\bar{a})=0$, from which it follows that $t=0$ or $c \bar{b}=\bar{a}$. If $t=0$, then $s=\bar{b}, c=\bar{a} b, f=d \bar{b}$; if $c \bar{b}=\bar{a}$, then $c=\bar{a} b, s=\bar{b}, t=0, f=d \bar{b}$; and

$$
\left\{\begin{array}{c}
p+q \bar{a}=\bar{d} \\
p a-q=\bar{e} \\
p d+q e=-1 \\
p b+q c=\bar{f}
\end{array}\right.
$$

By (3.45), similarly we have $p=0, q=-\bar{e}, d=-a e, f=d \bar{b}=-a \bar{b} e$. This implies that $A[\{1,2,2 m+1,2 m+2\} ;\{1,2,2 m+1,2 m+2\}]$ is of the following form,

$$
\left(\begin{array}{cccc}
1 & a & b & -a e \\
\bar{a} & -1 & \bar{a} b & e \\
\bar{b} & a \bar{b} & 1 & -a \bar{b} e \\
-\overline{a e} & \bar{e} & -\bar{a} b \bar{e} & -1
\end{array}\right) .
$$

In other words, if $a_{2 m+1,1}=\bar{b}$, then the $(2 m+1)^{t h}$ row vector of $A$ is a $\bar{b}$ multiple of $1^{s t}$ row vector; if $a_{2 m+2,2}=\bar{e}$, then the $(2 m+2)^{t h}$ row vector of $A$ is a $-\bar{e}$ multiple of $2^{\text {nd }}$ row vector, where $1 \leq m \leq \frac{n}{2}-1$. From this we have deduced that $A$ depends on only $(n-1)$ complex numbers with modulus at most 1 , for $n$ is even. 
When $n=2 k+1, k \in \mathbb{N}$, we may assume that there are $k(-1)^{\prime s},(k+1) 1^{\prime s}$ along the main diagonal line of $A$, and $\sigma(A)=\left\{\frac{1}{n}+\sqrt{\frac{n^{2}}{2}-\frac{1}{2 n}}, \frac{1}{n}, \ldots, \frac{1}{n}, \frac{1}{n}-\sqrt{\frac{n^{2}}{2}-\frac{1}{2 n}}\right\}$. Let $A^{\prime}=A-\frac{1}{n} I=\left(A^{\prime}\right)^{*} \in \mathscr{H}_{n}(\mathbb{C})$ with entries along the main diagonal of $A^{\prime}$ are $k\left(-1-\frac{1}{n}\right)^{\prime s},(k+1)\left(1-\frac{1}{n}\right)^{\prime s}$ and the other entries are complex numbers with modulus at most 1 . Since $\operatorname{rank}\left(A^{\prime}\right)=2$, we can apply the same strategy as above to find the structure of $A^{\prime}$, which will then yield the structure of $A$.

There are 3 types of invertible $2 \times 2$ principal submatrices of $A^{\prime}$

$$
\left(\begin{array}{cc}
1-\frac{1}{n} & a \\
\bar{a} & -1-\frac{1}{n}
\end{array}\right) \quad\left(\begin{array}{cc}
-1-\frac{1}{n} & a \\
\bar{a} & -1-\frac{1}{n}
\end{array}\right) \quad\left(\begin{array}{cc}
1-\frac{1}{n} & a \\
\bar{a} & 1-\frac{1}{n}
\end{array}\right) .
$$

$$
\text { type } 1 \quad \text { type } 2 \text { type } 3
$$

As $n \rightarrow \infty$, the determinants of the principal submatrices of type 2 and type 3 approach 0 . So we can determine the other row vectors as above by identifying the upper left invertible principal submatrix. From this we arrive at the structure of $A^{\prime}$ by study the principal submatrix

$$
\left(\begin{array}{cc|cc|c}
1-\frac{1}{n} & a & b & d & g \\
\bar{a} & -1-\frac{1}{n} & c & e & h \\
\hline \bar{b} & \bar{c} & 1-\frac{1}{n} & f & i \\
\bar{d} & \bar{e} & \bar{f} & -1-\frac{1}{n} & j \\
\hline \bar{g} & \bar{h} & \bar{i} & \bar{j} & 1-\frac{1}{n}
\end{array}\right) .
$$

Let the row vectors in the above submatrix be $\alpha, \beta, \gamma, \delta, \zeta$, and $\gamma, \delta, \zeta$, are combinations 
of $\alpha, \beta$ with combination parameters $(s, t),(p, q),(u, v)$, respectively. It follows that

$$
\left\{\begin{array}{c}
s\left(1-\frac{1}{n}\right)+t \bar{a}=\bar{b} \\
s a+t\left(-1-\frac{1}{n}\right)=\bar{c} \\
s d+t e=f \\
s g+t h=i
\end{array}\right.
$$

and

$$
\left\{\begin{array}{c}
p\left(1-\frac{1}{n}\right)+q \bar{a}=\bar{d} \\
p a+q\left(-1-\frac{1}{n}\right)=\bar{e} \\
p b+q c=\bar{f} \\
p d+q e=-1-\frac{1}{n} \\
p g+q h=j,
\end{array}\right.
$$

and

$$
\left\{\begin{array}{c}
u\left(1-\frac{1}{n}\right)+v \bar{a}=\bar{g} \\
u a+v\left(-1-\frac{1}{n}\right)=\bar{h} \\
u b+v c=\bar{i} \\
u d+v e=\bar{j} \\
u g+v h=1-\frac{1}{n} .
\end{array}\right.
$$

If $n=3$, we only have the first three equations in (3.46). These equations reduce to $2 \operatorname{Re}(a \bar{b} c)=2-\frac{4}{n}-\frac{1}{n^{2}}+\frac{1}{n^{3}}$ and $2 R e(a \overline{d e})=-\left(2+\frac{4}{n}-\frac{1}{n^{2}}-\frac{1}{n^{3}}\right)$.

As $n \rightarrow \infty, \operatorname{Re}(a \bar{b} c) \rightarrow 1$. So $a \bar{b} c=1, t=0, s=\bar{b}$; and $\operatorname{Re}(a \bar{d} e)=-1$, then $a \overline{d e}=-1, p=0, q=-\bar{e}$.

So if $n$ is odd, the entries of $A$ are determined by the same idea and $1,-1$ occurs along the diagonal of $A$ in pair, the last diagonal entry is 1 , and if $a_{2 m+1,1}=\bar{b}$, then 100 
the $(2 m+1)^{t h}$ row vector of $A$ is $\bar{b}$ multiple of $1^{\text {st }}$ row vector; if $a_{2 m+2,2}=\bar{e}$, then the $(2 m+2)^{t h}$ row vector of $A$ is $-\bar{e}$ multiple of $2^{\text {nd }}$ row vector, where $1 \leq m \leq \frac{n-1}{2}-1$, and the rule applies in the last row as same as the one in the $3^{\text {rd }}$ row. 


\subsubsection{Complex Hermitian Matrices with Entries of Modulus}

Let $A=\left[a_{i j}\right] \in \mathscr{H}_{n}(\mathbb{C}),\left\|a_{i j}\right\| \leq 1$, then $S P(A)=\lambda_{1}-\lambda_{n}$, if $\lambda_{1} \geq \lambda_{2} \geq$ $\cdots \geq \lambda_{n}$, all the eigenvalues of $A$. In fact $S P(A)=\lambda_{1}-\lambda_{n}=x^{*} A x-y^{*} A y=$ $e^{T}\left(A \circ \overline{\left(x x^{*}-y y^{*}\right)}\right) e$, where $x, y$ are the associated eigenvectors of $\lambda_{1}$ and $\lambda_{n}$. Now define $\tilde{A}=\left[\tilde{a_{i j}}\right], \tilde{a_{i j}}=\cos \left(-\alpha_{i j}\right)+i \sin \left(-\alpha_{i j}\right)$ where $B=\overline{\left(x x^{*}-y y^{*}\right)}=B^{*}=$ $\left(b_{i j}\right), b_{i j}=r_{i j}^{b}\left(\cos \left(\alpha_{i j}\right)+i \sin \left(\alpha_{i j}\right)\right)$ and $a_{i j}=r_{i j}^{a}\left(\cos \left(\beta_{i j}\right)+i \sin \left(\beta_{i j}\right)\right)$, then

$$
S P(A) \leq e^{T}(\tilde{A} \circ B) e=x^{*} \tilde{A} x-y^{*} \tilde{A} y \leq \lambda_{1}(\tilde{A})-\lambda_{n}(\tilde{A}),
$$

and

$$
S P(\tilde{A})=\sum_{1 \leq i \leq n} \tilde{a_{i i}} b_{i i}+2 R e \sum_{1 \leq i<j \leq n} \tilde{a_{i j}} b_{i j} .
$$

Observe that $A, \tilde{A}$, and $B$ are all Hermitian matrices. Thus $\tilde{a_{i i}} \in \mathbb{R}$ and $\left\|\tilde{a_{i j}}\right\|=1$ if and only if the spread of $\tilde{A}, S P(\tilde{A})$ is maximum.

Remark: According to Theorem 2.2.3, when $A=\left[a_{i j}\right] \in \mathscr{H}_{n},\left\|a_{i j}\right\|=1, S P(A) \leq$ $\sqrt{2\|A\|_{F}^{2}}=\sqrt{2} n$, if $n$ is even; and $S P(A) \leq \sqrt{2\|A\|_{F}^{2}-\frac{2}{n}|\operatorname{tr}(A)|^{2}} \leq \sqrt{2 n^{2}-\frac{2}{n}}$, if $n$ is odd.

Examples of some matrices $\tilde{A}=\left[\tilde{a_{i j}}\right] \in \mathscr{H}_{n},\left\|\tilde{a_{i j}}\right\|=1$, which attain the maximum spread are included below.

- $n=2: S P(\tilde{A})=2 \sqrt{2}$,

$$
\left(\begin{array}{cc}
1 & a \\
\bar{a} & -1
\end{array}\right) .
$$


- $n=3: S P(\tilde{A})=\sqrt{18-\frac{2}{3}}, \lambda_{1}=\frac{1}{3}+\sqrt{\frac{9}{2}-\frac{1}{6}}, \lambda_{2}=\frac{1}{3}, \lambda_{3}=\frac{1}{3}-\sqrt{\frac{9}{2}-\frac{1}{6}}$ if $\operatorname{tr}(\tilde{A})=1$ and $\operatorname{Re}(a \bar{b} c)=\frac{8}{27}$

$$
\left(\begin{array}{ccc}
1 & a & b \\
\bar{a} & 1 & c \\
\bar{b} & \bar{c} & -1
\end{array}\right)
$$

- $n=4: S P(\tilde{A})=4 \sqrt{2}, \lambda_{1}=-\lambda_{4}=2 \sqrt{2}, \lambda_{2}=\lambda_{3}=0$, if $\operatorname{tr}(\tilde{A})=0$ and

$$
\operatorname{Re}(d \bar{e} f+b \bar{c} f)=-\operatorname{Re}(a \bar{b} d+a \bar{c} e), 2 \operatorname{Re}(a \bar{b} d+a \bar{c} e)+\operatorname{Re}(a \bar{b} e \bar{f}+a \bar{c} d f+b \bar{c} d e)=3
$$

$$
\left(\begin{array}{cccc}
1 & a & b & c \\
\bar{a} & 1 & d & e \\
\bar{b} & \bar{d} & -1 & f \\
\bar{c} & \bar{e} & \bar{f} & -1
\end{array}\right) .
$$




\subsubsection{Skew Hermitian Matrices with Entries of Modulus No}

\section{More than 1}

Let $A=\left[a_{i j}\right] \in \mathscr{S} \mathscr{H}_{n}(\mathbb{C}),\left\|a_{i j}\right\| \leq 1$, which implies that $A^{*}=-A$ and $i A=$ $B=\left[b_{i j}\right] \in \mathscr{H}_{n}$, so by the results we have in the previous section on Hermitian matrices, when $S P(A)$ is maximum and attains the upper bound in Theorem 2.2.3 and $\operatorname{rank}(A)=2$, the structure of $A$ is as follows,

Case 1: If $n$ is even, $B[\{1,2,2 m+1,2 m+2\} ;\{1,2,2 m+1,2 m+2\}]$ is the following matrix

$$
\left(\begin{array}{cccc}
1 & a & b & -a e \\
\bar{a} & -1 & \bar{a} b & e \\
\bar{b} & a \bar{b} & 1 & -a \bar{b} e \\
-\bar{a} e & \bar{e} & -\bar{a} b \bar{e} & -1
\end{array}\right) .
$$

In other words, $1,-1$ occurs along the diagonal of $B$ in pairs, and if $b_{2 m+1,1}=\bar{b}$, then the $(2 m+1)^{t h}$ row vector of $B$ is $\bar{b}$ multiple of $1^{\text {st }}$ row vector; if $b_{2 m+2,2}=\bar{e}$, then the $(2 m+2)^{t h}$ row vector of $B$ is $-\bar{e}$ multiple of $2^{\text {nd }}$ row vector, where $1 \leq m \leq \frac{n}{2}-1$. Then we have the structure of $B$ which can be decided by $(n-1)$ complex numbers with modulus no more than 1, The same conclusion then holds for $A$.

Case 2: If $n$ is odd, entries of $B$ is decided by the same argument and 1, -1 occurs along the diagonal of $B$ in pairs, the last diagonal entry is 1 , and if $b_{2 m+1,1}=\bar{b}$, then the $(2 m+1)^{t h}$ row vector of $B$ is $\bar{b}$ multiple of $1^{\text {st }}$ row vector; if $b_{2 m+2,2}=\bar{e}$, 
then the $(2 m+2)^{t h}$ row vector of $B$ is $-\bar{e}$ multiple of $2^{\text {nd }}$ row vector, where $1 \leq m \leq \frac{n-1}{2}-1$, and the rule applied in the last row is same as the one in the $3^{\text {rd }}$ row.

Remark: According to Theorem 2.2.3, when $A=\left[a_{i j}\right] \in \mathscr{S} \mathscr{H}_{n},\left\|a_{i j}\right\|=1, S P(A) \leq$ $\sqrt{2\|A\|_{F}^{2}}=\sqrt{2} n$, if $n$ is even; and $S P(A) \leq \sqrt{2\|A\|_{F}^{2}-\frac{2}{n}|\operatorname{tr}(A)|^{2}} \leq \sqrt{2 n^{2}-\frac{2}{n}}$, if $n$ is odd.

Note: When $n$ is odd, for the structure we have studied,

$$
2 \operatorname{Im}(a \bar{b} c)=2-\frac{4}{n}-\frac{1}{n^{2}}+\frac{1}{n^{3}}=2 \operatorname{Im}(a \bar{g} h)
$$

and

$$
2 \operatorname{Im}(a \bar{d} e)=-\left(2+\frac{4}{n}-\frac{1}{n^{2}}-\frac{1}{n^{3}}\right) .
$$




\section{Chapter 4}

\section{Spectral Inclusions for Normal Matrices}

In this chapter, we review some literature on spectral inclusion regions, which concentrates on the relation between a normal matrix and its principal submatrices. This idea affords another avenue to the maximum spread of a certain matrix.

\subsection{New Spectral Inclusions for Normal Matrices}

We present some work on spectral inclusion regions for normal matrices [1], and then we find some connections between these spectral inclusion regions and the maximum spread for normal matrices.

Before we come to these conjectures, we define some notation which will be used throughout all this section.

Let $A=\left(a_{i j}\right)$ be an $n \times n$ complex matrix with spectrum $\sigma(A)=\left\{\lambda_{i}\right\}_{i=1}^{n}$. From this section, we denote by $A_{i}$ the $(n-1) \times(n-1)$ principal submatrix of $A$ obtained by deleting the $i$-th row and column from $A$ and let $\sigma\left(A_{i}\right)=\left\{\mu_{i j}\right\}_{j=1}^{n-1}$ be the spectrum 
of $A_{i}$, and, for $1 \leq i \leq n$, define

$$
r_{i}=\left[\sum_{j=1, j \neq i}^{n}\left|a_{i j}\right|^{2}\right]^{1 / 2}
$$

and let $D(\alpha, r)$ be the closed disc with radius $r \in \mathbb{R}^{+}$and center $\alpha \in \mathbb{C}$.

Conjecture 4.1.1 ([1]) For any $n \times n$ normal complex matrix $A$,

$$
\sigma(A) \subseteq \bigcup_{i=1}^{n} \bigcup_{j=1}^{n-1} D\left(\mu_{i j}, r_{i}\right)
$$

Recall the Geršchgorin classical disc theorem,

$$
\sigma(A) \subseteq \bigcup_{i=1}^{n} D\left(a_{i i}, \rho_{i}\right), \text { where } \rho_{i}=\sum_{j=1, j \neq i}\left|a_{i j}\right| \text { for } 1 \leq i \leq n .
$$

Note that (4.49) uses $n(n-1)$ discs centered at the eigenvalues of the principal submatrices $A_{i}$ of $A$, while (4.50) uses $n$ discs centered at the diagonal entries of $A$ but the related radii in (4.50) is bigger than those in (4.49) since $r_{i} \leq \rho_{i}$ for $1 \leq i \leq n$.

Conjecture 4.1.2 ([1]) Let $A$ be a singular $n \times n$ normal complex matrix. Then there exists $i \in\{1, \ldots, n\}$ such that

$$
\min _{\mu \in \sigma\left(A_{i}\right)}|\mu| \leq r_{i}
$$

It is easy to see that Conjecture 4.1 .1 is equivalent to Conjecture 4.1 .2 since in the first conjecture we can replace $A$ by $A^{\prime}=A-\lambda I$ for some $\lambda \in \sigma(A)$, then apply $A^{\prime}$ in the second conjecture.

Let $e_{1}, \ldots, e_{n}$ be the standard basis in $\mathcal{H}:=\mathbb{C}^{n}$ and denote by $P_{i}$ the orthogonal projection on $e_{i}^{\perp}$, so that $P_{i} \mathcal{H}=e_{i}^{\perp}$ and $P_{1}+\cdots+P_{n}=(n-1) I$. One has

$$
A_{i}=\left.P_{i} A P_{i}\right|_{P_{i} \mathcal{H}} \text { and }\left\|\left(I-P_{i}\right) A P_{i}\right\|_{F}=r_{i} \text { for } 1 \leq i \leq n .
$$


Clearly, if $A$ is a rank-one operator then $\|A\|_{F}=\|A\|$, where $\|\cdot\|$ denotes the operator norm. Here

$$
r_{i}=\left\|\left(I-P_{i}\right) A P_{i}\right\|_{F}=\left\|\left(I-P_{i}\right) A P_{i}\right\| \text { for } 1 \leq i \leq n \text {. }
$$

The following is another related conjecture from [1].

Conjecture 4.1.3 ([1]) Let $\left\{P_{i}\right\}_{i=1}^{n}$ be a family of rank $(n-1)$ projections in $n$ dimensional Hilbert space $\mathcal{H}$ such that $\left(I-P_{i}\right) \perp\left(I-P_{j}\right)$ for $1 \leq i \neq j \leq n$. For any singular normal operator $A \in L(\mathcal{H})$ one has

$$
\min _{1 \leq i \leq n}\left[\min _{\mu \sigma\left(A_{i}\right)}|\mu|-\left\|\left(I-P_{i}\right) A P_{i}\right\|\right] \leq 0
$$

where $A_{i}=\left.P_{i} A P_{i}\right|_{P_{i} \mathcal{H}}, 1 \leq i \leq n$

Evidently, Conjectures 4.1.1-4.1.3 are true for diagonal matrices. When $n=2$, these conjectures become precisely Geršchgorin's Disc Theorem and hence are true in this case. When $n=3$, we have the following theorem.

Theorem 4.1.4 ([1]) Conjecture 4.1.1-4.1.3 are true for $n=3$.

To prove it, we need Banach Spaces and to construct a surjective linear operator with surjective constant $M$ (see [1]).

There is a better covering conclusion when $A$ is a Hermitian matrix.

Theorem 4.1.5 ([1]) If $A$ is an $n \times n$ Hermitian matrix then

$$
\sigma(A) \subseteq \bigcup_{i=1}^{n} \bigcup_{j=1}^{n-1} D\left(\mu_{i j}, \frac{r_{i}}{\sqrt{n-1}}\right)
$$

so that $\sigma(A) \subseteq \bigcup_{i=1}^{n} \bigcup_{j=1}^{n-1}\left[\mu_{i j}-\frac{r_{i}}{\sqrt{n-1}}, \mu_{i j}+\frac{r_{i}}{\sqrt{n-1}}\right]$. 
Theorem 4.1.5 will follow the lemmas below.

Lemma 4.1.6 ([1]) Let $n \geq 2$ and $v=\left(v_{1}, \ldots, v_{n}\right)^{t} \in \mathbb{C}^{n}$ be such that at least two of its components are non-zero. Then

$$
\min _{1 \leq i \leq n} \frac{\left|v_{i}\right|}{\left[\sum_{j=1, j \neq i}^{n}\left|v_{j}\right|^{2}\right]^{1 / 2}} \leq \frac{1}{\sqrt{n-1}}
$$

Lemma 4.1.7 ([1]) If $A$ is an $n \times n$ singular Hermitian matrix then there exists $1 \leq i \leq n$ such that

$$
\min _{\mu \in \sigma\left(A_{i}\right)}|\mu| \leq \frac{r_{i}}{\sqrt{n-1}}
$$

Proof. Evidently, it is true if there is at least one $(n-1) \times(n-1)$ singular principal submatrices of $A$. Let $v=\left(v_{1}, \ldots, v_{n}\right)^{T} \in \mathbb{C}^{n} \backslash\{0\}$ and $A v=0$. If all but one component of $v$ are zero, assume that $v_{n} \neq 0$ then the $n^{\text {th }}$ column of $A$ is a zero vector. Hence there are at least $n-1$ singular principal submatrices of $A_{1}, \ldots, A_{n}$ and then the lemma is obvious in this situation. Now we assume that $v$ has at least two non-zero components. By Lemma 4.1.6, we have $v_{n} \neq 0$ and

$$
\frac{\left|v_{n}\right|}{\left[\sum_{j=1}^{n-1}\left|v_{j}\right|^{2}\right]^{1 / 2}} \leq \frac{1}{\sqrt{n-1}}
$$

Let

$$
v^{(n)}=\frac{\left(v_{1}, \ldots, v_{n-1}\right)^{T}}{\left[\sum_{j=1}^{n-1}\left|v_{j}\right|^{2}\right]^{1 / 2}} \in \mathbb{C}^{n-1} \backslash\{0\}
$$

so that $\left\|v^{(n)}\right\|_{2}=1$. From $A v=0$ we have

$$
A_{n} v^{(n)}=-\frac{v_{n}\left(a_{1 n}, \ldots, a_{n-1 n}\right)^{T}}{\left[\sum_{j=1}^{n-1}\left|v_{j}\right|^{2}\right]^{1 / 2}}
$$


Since both $A_{n}$ and $A$ are Hermitian it follows from the last identity and (4.51) that

$$
\begin{aligned}
\min _{\mu \in \sigma\left(A_{n}\right)}|\mu| & =\inf _{\|x\|_{2}=1}\left\|A_{n} x\right\|_{2} \leq\left\|A_{n} v^{(n)}\right\|_{2} \\
& \leq\left[\frac{\sum_{i=1}^{n-1}\left|a_{i n}\right|^{2}}{n-1}\right]^{1 / 2}=\left[\frac{\sum_{j=1}^{n-1}\left|a_{n j}\right|^{2}}{n-1}\right]^{1 / 2}=\frac{r_{n}}{\sqrt{n-1}}
\end{aligned}
$$

which proves the lemma.

Theorem 4.1.5 now follows by applying Lemma 4.1.7 to the $n \times n$ singular Hermitian matrices $A-\lambda I, \lambda \in \sigma(A)$.

Thus, Borcea describes the covering of eigenvalues for normal matrices, order $n \geq 3$, by discs generated from all its principal submatrices, order $(n-1)$. Applying Conjecture 4.1.1 (or Conjecture 4.1.2) to normal matrices, order $n \geq 3$, whose spreads are attained the maximum value, we will find the eigenvalues of these normal matrices are covered by the related $n(n-1)$ circles, since for these matrices, the corresponding rank is 2 , which means they are singular.

Lemma 4.1.8 For $a \in[-1,1), n \in N$, we have $\operatorname{round}\left(\frac{n-1}{a+3}\right)=\operatorname{round}\left(\frac{n}{a+3}\right)$ otherwise, $\operatorname{round}\left(\frac{n-1}{a+3}\right)=\operatorname{round}\left(\frac{n}{a+3}\right)-1$.

Lemma 4.1.8 shows that if $A \in S_{n}-\{a, 1\}$ and $A$ has the structure in (3.40), then its principal submatrices of order $(n-1)$, the maximum spread is attained for $A_{1}$ or $A_{n}$. Meanwhile, in this case, $A$ and its principal submatrices are all Hermitian, by the eigenvalue interlacing theorem and Theorem 4.1.5, we have the following conjecture.

Conjecture 4.1.9 $A \in S_{n}-\{a, 1\}$ and $A$ has the structure in (3.40), then

$$
S P(A) \leq \max \left\{S P\left(A_{1}\right), S P\left(A_{n}\right)\right\}+2 .
$$


For the matrix $A \in S S_{n}[-1,1]$, when the spread is maximum, then $A$ is of the form in Theorem 3.5.7. Then we have the following conjecture.

Conjecture 4.1.10 For the matrix $A \in S S_{n}[-1,1], \max \{S P(A)\}$ attains, then $A$ has the structure presented in Theorem 3.5.7, and

$S P(A) \leq \max \left\{S P\left(A_{1}\right)+2 \frac{\sqrt{s+t}}{\sqrt{n-1}}, S P\left(A_{r+1}\right)+2 \frac{\sqrt{r+t}}{\sqrt{n-1}}, S P\left(A_{r+s+1}\right)+2 \frac{\sqrt{s+r}}{\sqrt{n-1}}\right\}$ where $r, s, t$ are the corresponding order of the block zero matrix, and $r+s+t=n$.

There are many works focusing on the relation between the normal matrix and its principal submatrices. We introduce them in the next section. 


\subsection{Imbedding Conditions for Normal Matrices}

Given a complex $n \times n$ matrix $A$ and $(n-k) \times(n-k)$ matrix $B$, we say $B$ is imbeddable in $A$ or, equivalently, $B$ is a compression of $A$ if $B$ is a principal submatrix of $U^{*} A U$ for some $n \times n$ unitary matrix $U$.

The question, when an $(n-k) \times(n-k)$ normal matrix $B$ can be imbedded in an $n \times n$ normal matrix $A$, was studied for the first time 50 years ago by Ky Fan and Gordon Pall [5], who gave a complete answer for $k=1$. In 1984, Carlson and Marques de Sá [3] proved a theorem on the same problem. Later, in 1998, Ikramov and Elsner [12] studied the slightly different question of finding conditions under which an $(n-k) \times(n-k)$ normal matrix $B$ can be-nontrivially-dilated to an $n \times n$ normal matrix $A$, with special attention to the $k=1$ and $k=2$ cases.

Since the spread of a normal matrix is invariant under unitary similarity, we want to build up a connection among Borcea's conjectures, spread, and imbedding conditions for normal matrices.

The following theorems gives necessary and sufficient conditions on imbedding for normal matrices.

Theorem 4.2.1 ([5]) Let $A$ be an $n \times n$ normal matrix with eigenvalues $\alpha_{1}, \ldots, \alpha_{n}$, $B$ an $(n-1) \times(n-1)$ normal matrix with eigenvalues $\beta_{1}, \ldots, \beta_{n-1}$. Renumber the eigenvalues so that $\alpha_{j}=\beta_{j-1}, j=q+1, \ldots, n$ and $\alpha_{1}, \ldots, \alpha_{q}$ are each distinct from $\beta_{1}, \ldots, \beta_{q-1}$. Then $B$ is imbeddable in $A$ if and only if the $2 q-1$ points $\alpha_{1}, \ldots, \alpha_{q}, \beta_{1}, \ldots, \beta_{q-1}$ are collinear and the $\beta^{\prime} s$ separate the $\alpha^{\prime} s$ on that line. 
In the Hermitian case, using Cauchy's interlacing theorem, only the $n-1$ case is needed for induction in the general $n-k$ case, which means we just insert intermediate sequences of eigenvalues for a chain of matrices. But we can't apply the same strategy in the normal case, as shown by the following example in [5]. Take

$$
A=\operatorname{diag}(0,1, i, 1+i), \text { and } B=\frac{1}{10} \operatorname{diag}(5+8 i, 5+2 i) .
$$

Let

$$
V=\frac{1}{\sqrt{10}}\left(\begin{array}{cc}
1 & 2 \\
1 & -2 \\
2 & -1 \\
2 & 1
\end{array}\right)
$$

then $V^{*} V=I_{2}$ and $V^{*} A V=B$ so $B$ is imbeddable in $A$ and by Theorem 4.2.1, any normal matrix $C$ of order 3 imbeddable in $A$ must have two of $0,1, i, 1+i$ as characteristic roots and its third root on the segment joining the remaining two. But by Theorem 4.2.1, $B$ can not be imbedded in any such matrix $C$.

Carlson and Marques [3] proved a more general result on the same problem.

Theorem 4.2.2 Let $A$ be an $n \times n$ normal matrix with nonzero eigenvalues $\alpha_{1}, \ldots, \alpha_{n}$, satisfying $\gamma+\pi>\arg \alpha_{1} \geq \cdots \geq \arg \alpha_{n} \geq \gamma$ for some $\gamma \geq 0$. Let $B$ be an $(n-k) \times(n-k)$ normal principal submatrix of $A$. The eigenvalues $\beta_{1}, \ldots, \beta_{n-k}$ of $B$ may be ordered so that $\gamma+\pi>\arg \beta_{1} \geq \cdots \geq \arg \beta_{n-k} \geq \gamma$ and

$$
\arg \alpha_{j} \geq \arg \beta_{j} \geq \arg \alpha_{j+k}, j=1, \ldots, n-k
$$

João Filipe Queiró and António Leal Duarte [24] applied the idea of using the lexicographic order in $\mathbb{C}$ to mimic the Hermitian case on this problem. 
For real $\theta$, let $\leq_{\theta}$ be the total order with positive cone $e^{i \theta} H$, where

$$
H=\{a+i b: a>0, \text { or } a=0 \text { and } b>0\}
$$

So the lexicographic order is $\leq_{0}$, and we have

$$
\alpha \leq_{\theta} \beta \Leftrightarrow e^{-i \theta} \alpha \leq_{0} e^{-i \theta} \beta
$$

The condition $\alpha \geq_{\theta} \beta$ means that, when we sweep the plane with parallel lines orthogonal to the $\arg \theta$ direction, intersecting that direction before going to the $\arg (\theta+\pi)$ one, we find $\alpha$ before we find $\beta$ (in case of a tie, $\alpha$ is found to the right of $\beta$ ). The numbering of elements in decreasing sequences of course depends on $\theta$.

Now let $\theta \in \mathbb{R}$ be arbitrary, take an $n \times n$ normal matrix $A$, and let $\alpha_{1}, \ldots, \alpha_{n}$ be the eigenvalues of $A$, ordered so that $\alpha_{1} \geq_{\theta} \cdots \geq_{\theta} \alpha_{n}, v_{1}, \ldots, v_{n}$ be corresponding orthonormal eigenvectors of $A$. For $j=1, \ldots, n$, denote by $E_{j}$ and $E_{j}^{\prime}$ the subspaces spanned by $v_{1}, \ldots, v_{j}$ and $v_{j}, \ldots, v_{n}$, respectively. Then in similar circumstances $\max$ and min used in the $\leq_{\theta}$ sense, Queiró and Duarte [24] have the following min-max and interlacing theorems for normal matrices.

Theorem 4.2.3 For $j=1, \ldots, n$ we have

$$
\alpha_{j}=\min _{x \in E_{j},\|x\|=1} x^{*} A x=\max _{x \in E_{j}^{\prime},\|x\|=1} x^{*} A x
$$

They also proved that

$$
\alpha_{j}=\max _{\operatorname{dim} E=j,} \min _{x \in E,\|x\|=1} x^{*} A x=\min _{\operatorname{dim} E=n-j+1,} \max _{x \in E,\|x\|=1} x^{*} A x
$$

and the interlacing theorem for normal matrices. 
Theorem 4.2.4 Let $\theta$ be arbitrary and $A$ be an $n \times n$ normal matrix with eigenvalues $\alpha_{1} \geq_{\theta} \cdots \geq_{\theta} \alpha_{n}$. If $B$ is a principal $(n-k) \times(n-k)$ normal submatrix of $A$ with eigenvalues $\beta_{1} \geq_{\theta} \cdots \geq_{\theta} \beta_{n-k}$, we have

$$
\alpha_{j} \geq_{\theta} \beta_{j} \geq_{\theta} \alpha_{j+k}, j=1, \ldots, n-k .
$$




\subsection{The Spread of a Normal Matrix and Its Principal Sub- matrices}

There are many papers that focus on the issue of relating the eigenvalue spread of a matrix and its principal submatrices.

Thompson has a series of studies $[25,26,28]$ on this problem for a normal matrix and its principal submatrices. There are many other studies inspired by his research, like Johnson and Robinson [14, 15], and Nylen and Tam [22]. We mainly introduce Thompson's results.

Theorem 4.3.1 ([26]) Let $A$ be a Hermitian matrix with eigenvalues $\lambda_{1} \geq \cdots \geq \lambda_{n}$, and $A_{i}$ the principal submatrix of $A$ obtained by deleting row $i$ and column $i, i=$ $1, \ldots, n$. Then

$$
\sum_{i=1}^{n} S P\left(A_{i}\right) \geq(n-2) S P(A)
$$

The main tool used to approve it are the interlacing theorem (since the principal submatrices of the Hermitian matrix are all Hermitian), and adjugate matrix.

Then Thompson asserted a quadratic spread conjecture by abundant computer generated numerical evidence. He proved it is true for $n=3$.

Conjecture 4.3.2 ([26]) Let $A$ be a Hermitian matrix and $A_{i}$ are the principal submatrices. Then

$$
\sum_{i=1}^{n} S P\left(A_{i}\right)^{2} \geq(n-2) S P(A)^{2} .
$$


Later Nylen and Tam [22] showed that this conjecture is false for $n \times n$ Hermitian matrices when $n \geq 4$. However, it is true if the coefficient $n-2$ is replaced by some smaller value which is depended on $n$.

Thompson [27] also studied the generality of quadratic spread conjecture for Hermitian matrices to a quadratic spread conjecture for Hermitian matrix pencils.

Define a Hermitian matrix pencil $\lambda H-K$, where $\lambda$ is an indeterminate, and $H, K$ are $n \times n$ Hermitian matrices, and $H$ is positive definite. Let the roots of the pencil $\lambda H-K$ be $\lambda_{1}, \ldots, \lambda_{n}$. Then there exists a unitary matrix $V$ such that $V^{*} H^{-1 / 2} K H^{-1 / 2} V=\operatorname{diag}\left(\lambda_{1}, \ldots, \lambda_{n}\right)$. Set $T=H^{1 / 2} V$ and let $T^{-1 *}=\left[t_{i, j}\right]$, and take $f(\lambda)=\operatorname{det}(\lambda H-K)=\operatorname{det} H\left(\lambda-\lambda_{1}\right) \cdots\left(\lambda-\lambda_{n}\right), f_{i}(\lambda)=\operatorname{det}\left(\lambda H_{i}-K-i\right)$ as the pencil characteristic polynomial and the characteristic polynomial of the subpencil $\operatorname{det}\left(\lambda H_{i}-K_{i}\right)$, respectively. Then (see [27])

$$
f_{i}(\lambda)=\sum_{j=1}^{n}\left|t_{i, j}\right|^{2} \frac{f(\lambda)}{\lambda-\lambda_{j}}
$$

Thus

$$
\left[\begin{array}{c}
f_{1}(\lambda) \\
\vdots \\
f_{n}(\lambda)
\end{array}\right]=\mathscr{T}\left[\begin{array}{c}
\frac{f(\lambda)}{\lambda-\lambda_{1}} \\
\vdots \\
\frac{f(\lambda)}{\lambda-\lambda_{n}}
\end{array}\right],
$$

where the matrix $\mathscr{T}=\left[\left|t_{i, j}\right|^{2}\right]=\left[\tau_{i, j}\right]$ is nonnegative but not necessarily doubly stochastic.

Define $S P(f(\lambda))$ of a polynomial $f(\lambda)$ with real roots to be the difference of its largest root and the smallest one. Then he has the following quadratic spread inequality theorem. 
Theorem 4.3.3 ([27]) Let $\mathscr{T}$ be an $n \times n$ matrix, $n \geq 3$, with nonnegative entries and no zero rows or columns. Let $f(\lambda)$ be an $n^{\text {th }}$ degree polynomial with roots $\lambda_{1}, \ldots, \lambda_{n}$ all real, and use $\mathscr{T}$ and $f$ to define polynomials $f_{1}(\lambda), \ldots, f_{n}(\lambda)$ as in (4.52). Then a positive constant $c$ not dependent on the roots of $f(\lambda)$ exists such that

$$
\sum_{i=1}^{n}\left[S P\left(f_{i}(\lambda)\right)\right]^{2} \geq c\left[S P(f(\lambda)]^{2} .\right.
$$

A similar theorem is derived from a Hermitian matrix pencil when define the spread of the pencil $\lambda H-K$ as the separation between the largest and smallest of the roots of the pencil.

Theorem 4.3.4 ([27]) Let $H$ and $K$ by $n \times n$ Hermitian matrix with $H$ positive definite and $n \geq 3$. Then a positive constant $c=c(H)$ depending only on the eigenvalues of $H$ and not depending on $K$, exists such that

$$
\sum_{i=1}^{n}\left[S P\left(\lambda H_{i}-K_{i}\right)\right]^{2} \geq c[S P(\lambda H-K)]^{2}
$$




\section{Chapter 5}

\section{Conclusion}

In this chapter, we review the entire thesis and summarize our work on the maximum spread of certain normal matrices, and develop a plan for future research.

\subsection{Summary}

Research on the spread of a matrix started in the 1950's, and there is much literature on upper and lower bounds on the spread of some general normal matrices and simple graphs $[6,9,13,17 \sim 19,32]$. By Mirsky's Theorem, Theorem 2.2.3, we began a study on the maximum spread over a certain class of normal matrix whose entries are in an interval or a circle.

For a real normal matrix, if $A \in S_{n}[a, 1], a \in[-1,1)$ and $\operatorname{rank}(A)=2$, and if $S P(A)$ is maximum, then $A$ must be of the form shown in Theorem 3.4.1; we presented the maximum spread and the corresponding structure of matrix in Conjecture 3.4 .3 when $A \in S_{n}[a, b]$. For a real skew symmetric matrix, when $A=\left[a_{i j}\right] \in S S_{n}[-1,1]$, and $\operatorname{rank}(A)=2$, with $\left|a_{i j}\right| \leq 1,1 \leq i, j \leq n$, we have Theorem 3.5 .5 which presents 
sufficient and necessary conditions of the maximum spread and the related structure of the matrix.

For a complex normal matrix, when $A=\left[a_{i j}\right] \in \mathscr{H}_{n}(\mathbb{C}),\left\|a_{i j}\right\| \leq 1$, if $S P(A)$ attains the upper bound in Theorem 2.2.3 and $\operatorname{rank}(A)=2$, then $1,-1$ occurs consecutively along the diagonal line of the matrix $A$, while the other entries are determined by $(n-1)$ complex numbers with modulus no more than 1 . Moreover, $\left\|a_{i j}\right\|=1$ if and only if the spread of $A, S P(A)$ is maximum. A similar conclusion can be applied in the class of $A=\left[a_{i j}\right] \in \mathscr{S} \mathscr{H}_{n}(\mathbb{C}),\left\|a_{i j}\right\| \leq 1$, since $A^{*}=-A$ and $i A=B=\left[b_{i j}\right] \in \mathscr{H}_{n}$.

We have not shown more detail about the case of higher rank of $A$, a normal matrix with entries in some specifical range although we know the case of real symmetrical Mirsky matrix. But we have many numerical results shows $\operatorname{rank}(A)$ might be 2 when the maximum spread of this normal matrix attains. And it is more complex if we try to describe the pattern of these matrices with higher rank since there are many combinations related to the extreme (modulus) value of the entries of $A$ in the specific range.

There are many papers that focus on the relation between a normal matrix and its principal submatrices $[1,3,5,14,15,22,24 \sim 27]$. This array of research inspires us to continue to study the properties of spread of a normal matrix (with the entries in an interval or a circle) in terms of its principal submatrices. Finally, we presented Conjectures 4.1.9, 4.1.10. 


\subsection{Future Considerations}

In the future, I would like to prove Conjecture 3.4 .3 and explore the properties of spectral inclusion for a normal matrix, and make more accurate upper bounds on the spread of a normal matrix in term of the spreads of its principal submatrices.

I would like to investigate the spread property of matrix power, defined as $A^{K}=$ $\prod_{i=1}^{K} A$, especially, $A^{0}=I$. As we know, calculating high powers of matrices can be very time-consuming, but the complexity of the calculation can be dramatically decreased by using Cayley-Hamilton theorem, which takes advantage of an identity found using the matrices' characteristic polynomial and gives a much more effective equation for $A^{k}$. There is not that much literature focusing on the spread of powers of a normal matrix.

Complex symmetric matrices are not always normal. Since complex symmetry is a purely algebraic property, then it has no effect on the spectrum of the matrix. It means, for any given set of $n$ numbers,

$$
\lambda_{1}, \lambda_{2}, \ldots, \lambda_{n} \in \mathbb{C}
$$

there exists a complex symmetric $n \times n$ matrix $A$ whose eigenvalues are just the prescribed numbers(5.53); see, e.g., [11]. From Lemma 2.2.2, we want to concentrate on this topic of maximum spread for complex symmetric matrices and the associated matrices structure.

The spectral gap, difference between the maximum and minimum value of the 
spread, has been of interest to others for some time. Since the characteristic polynomial is a continuous function of the entries of the matrix, then the spread is continuous and then attains the lower bound and upper bound in a closed set. We would like to investigate this research further. 


\section{Chapter 6}

\section{Appendix}

\subsection{The Matlab Programs}

The $1^{\text {st }}$ program of Matlab aims to look for the maximum spread and the related the structure of the matrices which entries in the interval $[a, 1]$, and compare them with the parameters in Theorem 2.2.3.

function sprmax $(n k, a k)$

$$
n=n k ; a=a k ; b=-a k ; \quad \text { (transfer parameters) }
$$

if $a=0$ or $a=-1$ then generate one set values for the number of the block, $s_{0}$ and the related $S P$ in Theorem 2.2.3 and set the initial value of the loop 
$k a=\operatorname{round}(n /(3+a)) ; \operatorname{flag}_{a}=0$

$s a=\operatorname{sqrt}\left((a+3) *(a-1) * k a^{2}+2 * n *(1-a) * k a+n^{2}\right)$

otherwise generate two sets values

$k a=\operatorname{round}(n /(3+a)) ; k b=\operatorname{round}(n /(3+b)) ;$

$s a=\operatorname{sqrt}\left((a+3) *(a-1) * k a^{2}+2 * n *(1-a) * k a+n^{2}\right) ;$ flag $_{a}=0$

$s b=\operatorname{sqrt}\left((b+3) *(b-1) * k b^{2}+2 * n *(1-b) * k b+n^{2}\right) ; \operatorname{flag}_{b}=0$

process the loop of generating the random matrices

if $a \neq 0$ and $a \neq-1$

then generate $3 \times 10^{6}$ random real matrices $A a$ with entries $a, 1$ and $A b$ with entries

$-a, 1$

$A a=\operatorname{rand}(n) ; A b=\operatorname{rand}(n)$

for $i=1: n$

for $j=i: n$

if $A a(i, j) \leq 0.5$

$A a(i, j)=a ; A a(j, i)=a$

else

$A a(i, j)=1 ; A a(j, i)=1$

if $A b(i, j) \leq 0.5$

$A b(i, j)=b ; A b(j, i)=b$

else

$A b(i, j)=1 ; A b(j, i)=1$ 
calculate the spread values $s p r_{a}, s p r_{b}$ of matrices $A a, A b$

if $s p r_{a}>s a$

$\operatorname{flag}_{a}=1 ; A a, k a$

if $s p r_{b}>s b$

$\operatorname{flag}_{b}=1 ; A b, k b$

when the loop is stop check the value of flag.

if $f \operatorname{lag}_{a}=0$

$\operatorname{disp}\left(\left[{ }^{\prime} T h e\right.\right.$ default matrix, positive a.'])

if $\operatorname{flag}_{b}=0$

$\operatorname{disp}([$ 'The default matrix, negative a.'])

if $a=0$ or $a=-1$

then generate $3 \times 10^{6}$ random real matrices $A a$ with entries $a, 1$

$A a=\operatorname{rand}(n) ;$ for $i=1: n$

for $j=i: n$

if $A a(i, j) \leq 0.5$

$A a(i, j)=a ; A a(j, i)=a$

else

$A a(i, j)=1 ; A a(j, i)=1$; 
calculate the spread values $s p r_{a}, s p r_{b}$ of matrices $A a, A b$

if $s p r_{a}>s a$

$\operatorname{flag}_{a}=1 ; A a, k a$

when the loop is stop check the value of flag.

if $\operatorname{flag}_{a}=0$

$\operatorname{disp}\left(\left[{ }^{\prime} T h e\right.\right.$ default matrix, positive a.'])

output the data to the file.

The $2^{\text {nd }}$ program of Matlab aims to look for the matrices which entries in the interval $[a, 1]$ and satisfy the property of Mirsky's Theorem.

function Mirsky $(n k, a k)$

$$
n=n k ; a=a k ; b=-a k ; \text { transfer the parameters }
$$

process $3 \times 10^{6}$ loops of generating the random matrices $A a$ with entries $a, 1$ and $A b$

with entries $-a, 1$

$A a=\operatorname{rand}(n) ; A b=\operatorname{rand}(n)$

in every loop assign the entries of the matrices 
for $i=1: n$

for $j=i: n$

if $A a(i, j) \leq 0.5$

$A a(i, j)=a ; A a(j, i)=a$

otherwise $A a(i, j)=1 ; A a(j, i)=1$;

if $A b(i, j) \leq 0.5$

$A b(i, j)=b ; A b(j, i)=b$

otherwise $A b(i, j)=1 ; A b(j, i)=1$;

calculate the eigenvalues of $A a, A b$ and output to $l a, l b$

compare the other eigenvalues with the average value of the $1^{s t}$ and $n^{\text {th }}$ in $l a, l b$; if all the remaining eigenvalues are equal to the mean, then output the corresponding matrix. 


\section{Bibliography}

[1] Julius Borcea, On New Types of Spectral and Numerical Range Inclusions for Normal Matrices. Stockholm University. Preprint.

[2] A. Brauer and A. C. Mewborn, The greatest distance between two characteristic roots of a matrix, Duke Math. J. 26 (1959) $653-661$.

[3] D. Carlson and E. Marques de Sá, Generalized minimax and interlacing theorem, Linear Multilinear Algebra 15 (1984) 77 - 103.

[4] D. Cveković, M. Doob, and H. Sachs, Spectra of Graphs, Academic Press, New York (1979).

[5] Ky Fan and G. Pall, Imbedding conditions for Hermitian and normal matrices, Canad. J. Math. 9 (1957) 298 - 304.

[6] Yi-Zheng Fan, Jing Xu, Yi Wang, and Dong Liang, The Laplacian Spread of a Tree, Discrete Mathematics and Theoretical Computer Science, 10 (1) (2008) 79 86. 
[7] M. Fiedler, Algebraic connectivity of graphs, Czech. Math. J. 23 (98) (1973) 298 $-305$.

[8] G. Finke, R. E. Burkard, and F. Rendl, Quadratic assignment problems, Ann. Discrete Math. 31 (1987) $61-82$.

[9] D. A. Gregory, D. Hershkowitz, and S. J. Kirkland, The spread of the spectrum of a graph, Linear Algebra and its Applications 332-334 (2001) 23 - 35.

[10] R. Grone, C. R. Johnson, E. Marques de Sa, and H. Wolkowicz, Constrained ranges of sesquilinear forms, unpublished research report (1983), University of Waterloo, Waterloo, ON, Canada.

[11] Roger A. Horn and Charles R. Johnson, Matrix Analysis, Cambridge University Press (2005).

[12] K. D. Ikramov and L. Elsner, On normal matrices with normal principal submatrices, J. Math. Sci. 89 (1998) $1631-1651$.

[13] C. R. Johnson, Normality and the numerical range, Linear Algebra Appl. 15 (1976) $89-94$.

[14] C. R. Johnson, Numerical ranges of principal submatrices, Linear Algebra and its Applications 37 (1981) 23 - 34.

[15] C. R. Johnson and H. A. Robinson, Eigenvalue inequalities for principal submatrices, Linear Algebra and its Applications 37 (1981) 11 - 22. 
[16] C. R. Johnson, R. Kumar, and H. Wolkowicz, Lower bounds for the spread, Linear Algebra and its Applications 71 (1985) 161 - 173.

[17] X. Li, J. Zhang, and B. Zhou, The spread of unicyclic graphs with given size of maximum matchings, Journal of Mathematical Chemistry, 42 (2007) 775 - 788.

[18] Bolian Liu and Liu Mu-huo, On the spread of the spectrum of a graph, Discrete Mathematics 309 (9) (2009) 2727 - 2732.

[19] L. Mirsky, The spread of a matrix, Mathematika 3 (1956) 127 - 130.

[20] L. Mirsky, Inequalities for mormal and Hermitian matrices, Duke Math. J. 24 (1957) $591-599$.

[21] Vladimir Nikiforov, linear combinations of graph eigenvalues, Electronic Journal of Linear Algebra, 15 (2006) 329 - 336.

[22] Peter Nylen and Tin-Yau Tam, On the spread of a Hermitian matrix and a conjecture of Thompson, Linear and Multilinear Algebra, 37 (1994) 3 - 11.

[23] Miroslav M. Petrović, on graphs whose spectral spread does not exceed 4, Publications de l'institut mathématique, 34 (48) (1983) 169 - 174.

[24] João Filipe Queiró and António Leal Duarte, Imbedding conditions for normal matrices, Linear Algebra and its Applications 430 (2009) 1806 - 1811.

[25] R. C. Thompson, Principal submatrices of normal and Hermitian matrices, Illinois J. Math. 10 (1966) 296 - 308. 
[26] R. C. Thompson, The eigenvalue spreads of a Hermitian matrix and its principal submatrices, Linear and Multilinear Algebra 32 (1992) 327 - 333.

[27] R. C. Thompson, Root spreads for polynomials and Hermitian matrix pencils, Linear Algebra and its Applications 220 (1995) 419 - 433.

[28] R. C. Thompson, Principal submatrices of normal and Hermitian matrices VIII: Principal sections of a pair of forms, Rocky Mountain J. Math. 2 (1972) 97 - 110.

[29] H. Weyl, Inequalities between the two kinds of eigenvalues of a linear transformation, Proc. Nat. Acad. Sci. 35 (1949) 408 - 411.

[30] H. Weyl, Über beschränkte quadratische Formen, deren Differenz vollsteig ist, Rend. Circ. Mat. Palermo 27 (1909) 373 - 392.

[31] H. Wolkowicz and G. P. H. Styan, Bounds for eigenvalues using traces, Linear Algebra Appl. 29 (1980) 471 - 506.

[32] H. Wolkowicz and G. P. H. Styan, More bounds for eigenvalues using traces, Linear Algebra Appl. 31 (1980) 1 - 17.

[33] X. Zhan, Extremal eigenvalues of real symmetric matrices with entries in an interval, SIAM J. Matrix Anal. Appl. 27 (2006) 851 - 860. 